PHYSICAL REVIEW D 92, 072005 (2015)

\title{
Measurements of the top quark branching ratios into channels with leptons and quarks with the ATLAS detector
}

\author{
G. Aad et al. ${ }^{*}$ \\ (ATLAS Collaboration) \\ (Received 17 June 2015; published 19 October 2015)
}

\begin{abstract}
Measurements of the branching ratios of top quark decays into leptons and jets using events with $t \bar{t}$ (top antitop) pairs are reported. Events were recorded with the ATLAS detector at the LHC in $p p$ collisions at a center-of-mass energy of $7 \mathrm{TeV}$. The collected data sample corresponds to an integrated luminosity of $4.6 \mathrm{fb}^{-1}$. The measured top quark branching ratios agree with the Standard Model predictions within the measurement uncertainties of a few percent.
\end{abstract}

DOI: $10.1103 /$ PhysRevD.92.072005

PACS numbers: $14.65 . \mathrm{Ha}$

\section{INTRODUCTION}

In the Standard Model (SM), $100 \%$ of the top quark decays contain a $W$ boson and a down-type quark. Measurements of the ratio of top branching fractions $B(t \rightarrow W+b$-quark $) / B(t \rightarrow W+$ down-type quark) [1] and of single top production [2-4] have shown that more than $95 \%$ of the decays are to a $W$ boson and a $b$-quark. In the SM the branching ratio to the different leptons is the same since the decay proceeds via a $W$ boson, but in models of new physics, e.g. supersymmetry (SUSY), final states with $\tau$ leptons can be enhanced or suppressed [5]; thus measuring the inclusive cross section using final states with $\tau$ leptons can be a good probe for new physics. The measured values of the top quark branching ratios will deviate with respect to the SM predictions if the data sample selected to extract $t \bar{t}$ events contains final states without two $W$ bosons. Examples of processes that would cause deviations include events with a top quark decaying to charged Higgs boson or with SUSY particles decaying to the supersymmetric partner of the $\tau$ lepton $(\tilde{\tau})$. Limits on the top quark branching ratio to a charged Higgs boson and a $b$-quark have been published by the CDF [6], D0 [7,8], ATLAS [9,10] and CMS [11] collaborations. Another example of a final state that can change the observed branching ratios is the pair production of supersymmetric partners of the top quark $(\tilde{t})$ decaying into $b \nu_{\tau} \tilde{\tau}$ followed by the $\tilde{\tau}$ decay into a $\tau$ lepton and the gravitino, predicted by gauge-mediated SUSY breaking models [12].

This article presents the first direct measurement of the top quark semileptonic and all-hadronic branching ratios. The branching ratios can be more sensitive probes of

*Full author list given at the end of the article.

Published by the American Physical Society under the terms of the Creative Commons Attribution 3.0 License. Further distribution of this work must maintain attribution to the author(s) and the published article's title, journal citation, and DOI. deviations from SM expectations than measuring cross sections in different channels, because of cancellation of systematic uncertainties. The large number of $t \bar{t}$ pairs produced at the LHC provides an opportunity to measure top quark branching ratios with high precision. These top quark branching ratios are expected to be determined by the $W$ boson branching ratios, which have been measured at LEP [13] to be in good agreement with the SM expectations [14]. Observing any deviation would be an indication of non-SM processes contributing to final states dominated by $t \bar{t}$ production. This article also presents a measurement of the inclusive $t \bar{t}$ cross section using events with an isolated charged lepton ( $\mu$ or $e$ ) and a $\tau$ lepton decaying hadronically $\left(\tau_{\text {had }}\right)$. Previous measurements of the cross section at $\sqrt{s}=7 \mathrm{TeV}$ in this channel have been published by the ATLAS and CMS collaborations $[15,16]$.

The analysis uses the full data sample, $4.6 \mathrm{fb}^{-1}$, collected by the ATLAS experiment at the LHC from $p p$ collisions at $\sqrt{s}=7 \mathrm{TeV}$ between March and November 2011. Kinematic selection criteria are applied that require one or both of the top and antitop quarks to decay into a final state with one isolated lepton and a jet. At least one jet in the event must be tagged as originating from a $b$-quark ( $b$-tag). Seven mutually exclusive final states are used in this analysis: $e+$ jets, $\mu+$ jets, $e e+$ jets, $\mu \mu+$ jets, $e \mu+$ jets, $e \tau_{\text {had }}+$ jets and $\mu \tau_{\text {had }}+$ jets. Branching ratios for semileptonic and purely hadronic top quark decays are obtained by combining these seven final states assuming that only SM processes contribute to the background and the top branching ratios to leptons and jets add up to one.

\section{ANALYSIS OVERVIEW}

Data samples enriched with $t \bar{t}$ events are selected by means of criteria that are designed to accept two $W$ bosons and at least one $b$-quark. In every event, either an electron or a muon is required, with the aim to select $W \rightarrow \ell \nu$, where $\ell$ stands for either $e$ or $\mu$. The $\ell$ may be produced directly in $W \rightarrow \ell \nu$ boson decays or indirectly in $W \rightarrow \tau \nu$ 
decays. Separate event channels are classified depending on the decay of a second $W$ boson: $W \rightarrow$ jets for $\ell+$ jets, $W \rightarrow \ell \nu$ for $\ell \ell^{\prime}+$ jets, or $W \rightarrow \tau_{\text {had }} \nu$ for $\ell \tau_{\text {had }}+$ jets. Since the analysis does not distinguish electrons or muons that originate from a $\tau$ lepton decay from those that come from direct $W \rightarrow e \nu$ and $W \rightarrow \mu \nu$ decays, both are included in the $W \rightarrow \ell \nu$ decays. The branching ratios are measured by taking ratios of the number of $t \bar{t}$ events extracted from the three channels; thus an important aspect of the event selection is to use similar criteria for the object selection in all final states, so as to allow the cancellation of systematic uncertainties in the ratios. Another important criterion is to ensure that no event contributes to more than one channel. The channel with the largest background and smallest number of signal events is that containing $\ell \tau_{\text {had }}+$ jets; thus the event selection and analysis were optimized to reduce the uncertainty in that channel (see Sec. V).

The number of $t \bar{t}$ events in a given channel is extracted by fitting background and signal templates to data distributions. The template shapes are fixed while their normalizations are allowed to vary. The signal templates are derived from $t \bar{t}$ Monte Carlo (MC) simulation, which assumes that the top quark decays to a $W$ boson and a $b$-quark with a $100 \%$ branching ratio. This assumption affects the shape of the signal templates, and if it is not valid for the selected data, the measured branching ratios will deviate from the SM prediction. The amount of background varies significantly in each channel. It is almost negligible in the $e \mu+$ jets channel and larger than the signal in the $\ell \tau_{\text {had }}+$ jets channels. In the $\ell+$ jets channels, three invariant masses from two- and three-jet systems and a transverse mass distribution are fitted, as described in detail in Sec. VI, while in the $\ell \ell^{\prime}+$ jets channels the dilepton effective mass distributions from two different missing transverse momentum $\left(E_{\mathrm{T}}^{\mathrm{miss}}\right)$ regions are used (see Sec. VII). Because of the much larger background, which originates from jets misidentified as $\tau$ leptons, a very different approach is taken in the $\ell \tau_{\text {had }}+$ jets channel. Instead of fitting a kinematic distribution, the quantity fitted is a boosted decision tree (BDT) output [17], a multivariate discriminant that separates jets from $\tau$ leptons decaying to hadrons (see Sec. VIII).

The details of how the inclusive production cross section and branching ratios are derived from the number of $t \bar{t}$ events obtained from each channel are discussed in Sec. IX. The systematic uncertainties of the measurements are estimated by varying each source of systematic uncertainty by $\pm 1 \sigma$ in templates derived from MC simulation and fitting all the distributions with the new templates (see Sec. X). The final results are given in Sec. XI.

\section{ATLAS DETECTOR}

The ATLAS detector [18] at the LHC covers nearly the entire solid angle around the collision point. ${ }^{1}$ It consists of an inner tracking detector surrounded by a thin superconducting solenoid, electromagnetic (EM) and hadronic calorimeters, and an external muon spectrometer incorporating three large superconducting toroid magnet assemblies. The inner tracking detector provides tracking information in a pseudorapidity range $|\eta|<2.5$. The liquid-argon (LAr) EM sampling calorimeters cover a range of $|\eta|<3.2$ with fine granularity. An iron/scintillator tile calorimeter provides hadronic energy measurements in the central rapidity range $(|\eta|<1.7)$. The endcap and forward regions are instrumented with LAr calorimeters for both the EM and hadronic energy measurements covering $|\eta|<4$.9. The muon spectrometer provides precise tracking information in a range of $|\eta|<2.7$.

In 2011, ATLAS used a three-level trigger system to select events. The level-1 trigger is implemented in hardware using a subset of detector information to reduce the event rate to less than $75 \mathrm{kHz}$. This is followed by two software-based trigger levels, namely level-2 and the event filter, which together reduce the event rate to about $300 \mathrm{~Hz}$ recorded for analysis.

\section{DATA AND MONTE CARLO SAMPLES}

The present measurements use collision data with a center-of-mass energy of $\sqrt{s}=7 \mathrm{TeV}$ taken in 2011 and selected with a single-electron or a single-muon trigger. Taking into account selection criteria for good data quality, the total integrated luminosity for the analyzed data sample is $4.6 \mathrm{fb}^{-1}$.

The $t \bar{t}$ signal is modeled using the POWHEG $[19,20]$ event generator, interfaced to PYTHIA6 (v6.421) [21] with the Perugia $2011 \mathrm{C}$ tune [22] for showering and hadronization, setting the top quark mass to $172.5 \mathrm{GeV}$ and using the next-to-leading-order (NLO) parton distribution function (PDF) set CTEQ66 [23]. The $t \bar{t}$ production cross section used in the simulation is normalized to $177 \mathrm{pb}$ as obtained from next-to-next-to-leading-order (NNLO) plus next-tonext-to-leading-logarithm (NNLL) calculations [24].

The calculation of the backgrounds uses MC simulations of $W / Z$ production with multiple jets (matrix elements for the jets production include light quarks, $c, \bar{c}, c \bar{c}, b \bar{b})$, single-top-quark, and diboson $(W W, W Z, Z Z)$ events. Single-top-quark events were generated using MC@NLO (v4.01) [25] interfaced with HERWIG (v6.520) [26] and JIMMY (v4.31) [27] to model parton showering,

\footnotetext{
${ }^{1}$ ATLAS uses a right-handed coordinate system with its origin at the nominal interaction point in the center of the detector and the $z$ axis along the beam pipe. The $x$ axis points to the center of the LHC ring, and the $y$ axis points upwards. The azimuthal angle $\phi$ is measured around the beam axis and the polar angle $\theta$ is the angle from the beam axis. The pseudorapidity is defined as $\eta=-\ln [\tan (\theta / 2)]$. The distance $\Delta R$ in $\eta-\phi$ space is defined as $\Delta R=\sqrt{(\Delta \phi)^{2}+(\Delta \eta)^{2}}$. The transverse momentum and energy are defined as $p_{\mathrm{T}}=p \sin \theta$ and $E_{T}=E \sin \theta$, respectively.
} 
hadronization, and the underlying-event using PDF set CT10 [28]. $W+$ jets events with up to five partons and $Z+$ jets events with $m\left(\ell^{+} \ell^{-}\right)>40 \mathrm{GeV}$ and up to five partons were generated by ALPGEN (v2.13) [29] interfaced to HERWIG plus JIMMY and the CTEQ6L1 [30] PDF set. The MLM matching scheme [31] of the ALPGEN generator is used to remove overlaps between matrixelement and parton-shower products. Diboson events were generated using HERWIG plus JIMMY and the MRSTMcal PDF set [32]. Scale factors are applied to each process to match next-to-leading-order predictions. The $\tau$ decays are handled by TAUOLA [33].

All samples of simulated events include the effect of multiple $p p$ interactions in the same and neighboring bunch crossings (pile-up). On average, nine minimum-bias events are overlaid on all simulated events to match the pile-up conditions in data. The average number of $p p$ collisions in a bunch crossing $(\langle\mu\rangle)$ depends on the instantaneous luminosity, which increased over time; $\langle\mu\rangle$ varied from 5 at the beginning of the run period to approximately 18 at the end. The events are reweighted in order to make the distribution of the average number of interactions per bunch crossing match the one observed in data. All MC events are simulated with a detailed GEANT4-based detector simulation $[34,35]$ and are reconstructed with the same algorithms as used in data.

\section{EVENT SELECTION}

Events are selected using a single-muon trigger with a $p_{\mathrm{T}}$ threshold of $18 \mathrm{GeV}$ or a single-electron trigger with an $E_{\mathrm{T}}$ threshold of $20 \mathrm{GeV}$, rising to $22 \mathrm{GeV}$ during periods of high instantaneous luminosity. The $p_{\mathrm{T}}$ and $E_{\mathrm{T}}$ criteria used in the further analysis guarantee a high and constant trigger efficiency. The same triggers and reconstructed object definitions are applied to all channels.

Muon candidates are selected using tracks from the inner detector matched with tracks in the muon spectrometer [36]. They are required to have $p_{\mathrm{T}}>20 \mathrm{GeV}$ and $|\eta|<2.5$ and to satisfy criteria designed to reduce the muon misidentification probability. The muon must have a longitudinal impact parameter $\left(z_{0}\right)$ with respect to the primary vertex of less than $2 \mathrm{~mm}$. In addition, to suppress muons from heavy-quark decays, muons must pass the isolation cuts: the calorimeter energy in a cone of size $\Delta R=0.2$ around the muon track must be less than $4 \mathrm{GeV}$, and the scalar sum of the $p_{\mathrm{T}}$ of the tracks reconstructed in the inner tracker in a cone of $\Delta R=0.3$ around the muon track must be less than $2.5 \mathrm{GeV}$. If a muon overlaps within a cone of $\Delta R=0.4$ with an electron candidate or with a jet, as defined below, it is not considered to be isolated.

Electron candidates are required to satisfy cuts on calorimeter and tracking variables to separate isolated electrons from jets [37]. Electrons must fall into the region $\left|\eta_{\text {cluster }}\right|<2.47$, where $\left|\eta_{\text {cluster }}\right|$ is the pseudorapidity of the calorimeter energy cluster associated with the electron, excluding the transition region between the barrel and endcap calorimeters at $1.37<\left|\eta_{\text {cluster }}\right|<1.52$, and have $E_{\mathrm{T}}>25 \mathrm{GeV}$. The electrons must also pass an $E_{\mathrm{T}}$ isolation cut within a cone of $\Delta R=0.2$ derived for $90 \%$ efficiency along with a $p_{\mathrm{T}}$ isolation cut within a cone of $\Delta R=0.3$ derived for $90 \%$ efficiency for prompt electrons from $Z \rightarrow$ $e^{+} e^{-}$events. The electron must have $z_{0}$ with respect to the primary vertex of less than $2 \mathrm{~mm}$. Finally, if the electron lies within a cone of $\Delta R=0.4$ around the muon or between $0.2<\Delta R \leq 0.4$ around a jet as defined below, the object is considered to be a muon or a jet, respectively.

Jets are reconstructed from clustered energy deposits in the calorimeters using the anti- $k_{t}$ [38] algorithm with a radius parameter $R=0.4$. Jets are required to have a transverse momentum $p_{\mathrm{T}}>25 \mathrm{GeV}$ and to be in the pseudorapidity range $|\eta|<2.5$. The summed scalar $p_{\mathrm{T}}$ of tracks associated with the jet and associated with the primary vertex is required to be at least $75 \%$ of the summed $p_{\mathrm{T}}$ of all tracks associated with the jet [39]. Any jet close to a good electron, as defined above, is considered to be an electron if it lies within a cone of $\Delta R=0.2$ around the electron. Missing transverse momentum $\left(E_{\mathrm{T}}^{\mathrm{miss}}\right)$ is the magnitude of the vector sum of the $x$ and $y$ components of the cluster energy in the calorimeters. Each cluster is calibrated according to which type of high- $p_{\mathrm{T}}$ object it is matched to, either electrons, jets, muons or photons.

Jets containing $b$-hadrons ( $b$-jets) are identified (b-tagged) with a multivariate discriminant that exploits the long lifetimes, high masses and high decay multiplicities of $b$-hadrons. It makes use of track impact parameters and reconstructed secondary vertices. An operating point corresponding to an average efficiency of $70 \%$ and an average mistag rate for light-quark jets of $0.8 \%$ is used [40].

$\tau$ candidates are reconstructed using calorimeter jets as seeds. These seed jets are calibrated with the local calibration (LC) scheme [41,42]. The $\tau$ candidate must have $E_{\mathrm{T}}^{\tau}>20 \mathrm{GeV},\left|\eta_{\tau}\right|<2.3$, and only one track with $p_{\mathrm{T}}>4 \mathrm{GeV}$ associated with the $\tau$ candidate $(77 \%$ of hadronic $\tau$ decays have only one track). The charge of the $\tau$ candidate is given by the charge of the associated track. Candidates with higher track multiplicity are not used as they do not improve the precision of the measurement because of much larger associated systematic uncertainties. The analysis makes use of a BDT for $\tau$ identification, a cut-based multivariate algorithm that optimizes signal and background separation [17].

The $\tau$ candidates that overlap within $\Delta R<0.4$ of a $b$-tagged jet, a loose muon, ${ }^{2}$ or an electron, ${ }^{3}$ are rejected and kept as jets or electrons. To remove the remaining

\footnotetext{
${ }^{2}$ Loose muons are selected with all requirements described in Sec. V for good muons, except $p_{\mathrm{T}}^{\mu}>4 \mathrm{GeV}$ and no isolation requirements are applied.

${ }^{3}$ These electrons are selected with all requirements described in Sec. V for good electrons, but electrons with $E_{\mathrm{T}}>20 \mathrm{GeV}$ are considered.
} 
electrons misidentified as $\tau$ candidates a medium BDT $\left(\mathrm{BDT}_{e}\right)$ electron veto is applied. $\mathrm{BDT}_{e}$ is a BDT trained to distinguish electrons and $\tau$ leptons using a $Z \rightarrow \tau \tau \mathrm{MC}$ sample as signal and a $Z \rightarrow \ell \ell \mathrm{MC}$ sample as background. The $\mathrm{BDT}_{e}$ uses four variables; the two most powerful being the ratio of high-threshold to low-threshold track hits in the transition radiator and the ratio of energy deposited in the EM calorimeter to the total energy deposited in the calorimeter. The medium working point corresponds to $85 \%$ efficiency for $Z \rightarrow \tau \tau$, Ref. [43]. The additional rejection factor for electrons after removing isolated electrons that overlap with $\tau$ candidates is 60 . In addition, a muon veto that compares the track momentum in $\tau$ candidates with the energy deposited in the electromagnetic calorimeter is required to further reduce the muon background. It is tuned to $96 \%$ efficiency on signal (62\% on background after overlap removal). A BDT to reject hadronic jets faking $\tau$ leptons, $\mathrm{BDT}_{j}$, is trained with $\tau$ leptons from a $Z \rightarrow \tau \tau \mathrm{MC}$ sample as signal and jets from data, selected from events with at least two jets, as background. The $\mathrm{BDT}_{j}$ uses eight variables, the most sensitive is the fraction of energy deposited in the region $\Delta R<0.1$ with respect to all energy deposited in the region $\Delta R<0.2$ around the $\tau$ candidate. Details of the $\mathrm{BDT}_{e}$ and $\mathrm{BDT}_{j}$ input variables and performance are given in Ref. [43].

The event selection requirements common to all channels are a primary vertex with at least five associated tracks with $p_{\mathrm{T}}>400 \mathrm{MeV}$, at least one isolated high- $p_{\mathrm{T}}$ muon $\left(p_{\mathrm{T}}>20 \mathrm{GeV}\right)$ and/or isolated high- $p_{\mathrm{T}}$ electron $\left(p_{\mathrm{T}}>25 \mathrm{GeV}\right)$, at least two jets with $p_{\mathrm{T}}>25 \mathrm{GeV}$, and at least one of them tagged as a $b$-jet. In addition, there are requirements specific to each channel. For the $\ell+$ jets channels the isolated-muon $p_{\mathrm{T}}$ threshold is raised from $20 \mathrm{GeV}$ to $25 \mathrm{GeV}$ to reduce the multijet background and exactly one isolated $\ell$ is required. The minimum number of jets with $p_{\mathrm{T}}>25 \mathrm{GeV}$ is raised to four. Events with $\tau$ candidates are removed. Removing events with $\tau$ candidates from the $\ell+$ jets channel results in an efficiency loss of $8.5 \%$. For the $\ell \ell^{\prime}+$ jets channels, events are required to have exactly two isolated $\ell$ with oppositesign charges and $E_{\mathrm{T}}^{\mathrm{miss}}>30 \mathrm{GeV}$. For the $\ell \tau_{\text {had }}+$ jets channels, exactly one isolated $\ell, E_{\mathrm{T}}^{\text {miss }}>30 \mathrm{GeV}$, and at least one $\tau$ candidate are required. In addition the $\ell$ and the $\tau$ candidate must have opposite charge. The $\tau$ candidates that do not satisfy these requirements are kept as jets. The thresholds for lepton $p_{\mathrm{T}}$, jet $p_{\mathrm{T}}$ and $E_{\mathrm{T}}^{\text {miss }}$ were optimized for the $\ell \tau_{\text {had }}+$ jets channel for maximum signal significance by means of a search in parameter space.

\section{SINGLE-LEPTON + JETS CHANNEL}

Three different classes of events contribute as a background to the $t \bar{t} \rightarrow \ell+$ jets channel:

(1) events with one isolated $\ell$ originating from processes with one true lepton ( $W$ boson decay);
(2) events with one jet misidentified as an isolated lepton and no other isolated lepton reconstructed;

(3) events with one isolated lepton originating from processes with multiple true leptons but only one isolated lepton reconstructed.

The number of $t \bar{t} \rightarrow \ell+$ jets events is extracted by fitting distributions of four invariant mass variables with templates for signal and backgrounds. The following mass variables provide good discrimination between signal and background:

(1) $m_{j j}$ : invariant mass of the two highest- $p_{\mathrm{T}}$ jets not designated as $b$-jets;

(2) $m_{b 1 j j}$ : invariant mass of the leading $b$-jet and the jets used to calculate $m_{j j}$;

(3) $m_{b 2 j j}$ : invariant mass of the subleading $b$-jet and the jets used to calculate $m_{j j}$;

(4) $m_{\mathrm{T}}$ : transverse mass of $\ell$ and the $E_{\mathrm{T}}^{\text {miss }}, m_{\mathrm{T}}\left(\ell, E_{\mathrm{T}}^{\text {miss }}\right)=$ $\sqrt{\left(E_{\mathrm{T}}^{\ell}+E_{\mathrm{T}}^{\mathrm{miss}}\right)^{2}-\left(p_{x}^{\ell}+E_{x}^{\mathrm{miss}}\right)^{2}-\left(p_{y}^{\ell}+E_{y}^{\mathrm{miss}}\right)^{2}}$.

If an event has only one jet tagged as a $b$-jet, the highest$p_{\mathrm{T}}$ jet that is not tagged is assumed to be a second $b$-jet. A few observations motivate the choice of mass distributions for the fit. The presence of a $W$ boson decaying to a pair of quarks leads to an $m_{j j}$ distribution that peaks at the $W$ boson mass. The presence of a top quark decaying to $W(\rightarrow q q)+b$ will produce $m_{b 1 j j}$ and $m_{b 2 j j}$ distributions that peak at the top quark mass. The presence of a $W$ boson decaying to $\ell+\nu$ manifests itself as a Jacobian peak in the $m_{\mathrm{T}}$ distribution when there are no additional high- $p_{\mathrm{T}}$ neutrinos in the event.

\section{A. Background templates}

The main backgrounds in the $\ell+$ jets channel are from $W(\rightarrow \ell \nu)+$ jets and other $t \bar{t}$ final states. There are also smaller contributions from single top, $Z(\rightarrow \ell \ell)+$ jets (with one lepton not identified) and multijet processes with one jet misidentified as a lepton. Background templates are derived from the MC simulations in all cases except multijet processes. The multijet background is very difficult to simulate due to the need for a very large sample and the fact that MC models do not reproduce that background well. Instead it is derived from a control data sample with nonisolated electrons and muons, keeping all other selection criteria the same. The distributions of a small expected contribution from $t \bar{t}$ is subtracted from the multijet control sample.

Figure 1 shows the $m_{j j}, m_{b 1 j j}, m_{b 2 j j}$ and $m_{\mathrm{T}}$ distributions predicted by $\mathrm{MC}$ simulation and normalized to unity for $W+$ jets, $Z+$ jets, and $t \bar{t} \rightarrow \ell+$ jets events. It also shows these distributions for multijet events derived from the control data sample. The distributions from other $t \bar{t}$ channels are not shown as that background is normalized following the $\mathrm{MC}$ prediction of the ratio to the number of $t \bar{t} \rightarrow \ell+$ jets events. The figure demonstrates that the shape of all the invariant mass distributions from jets are 

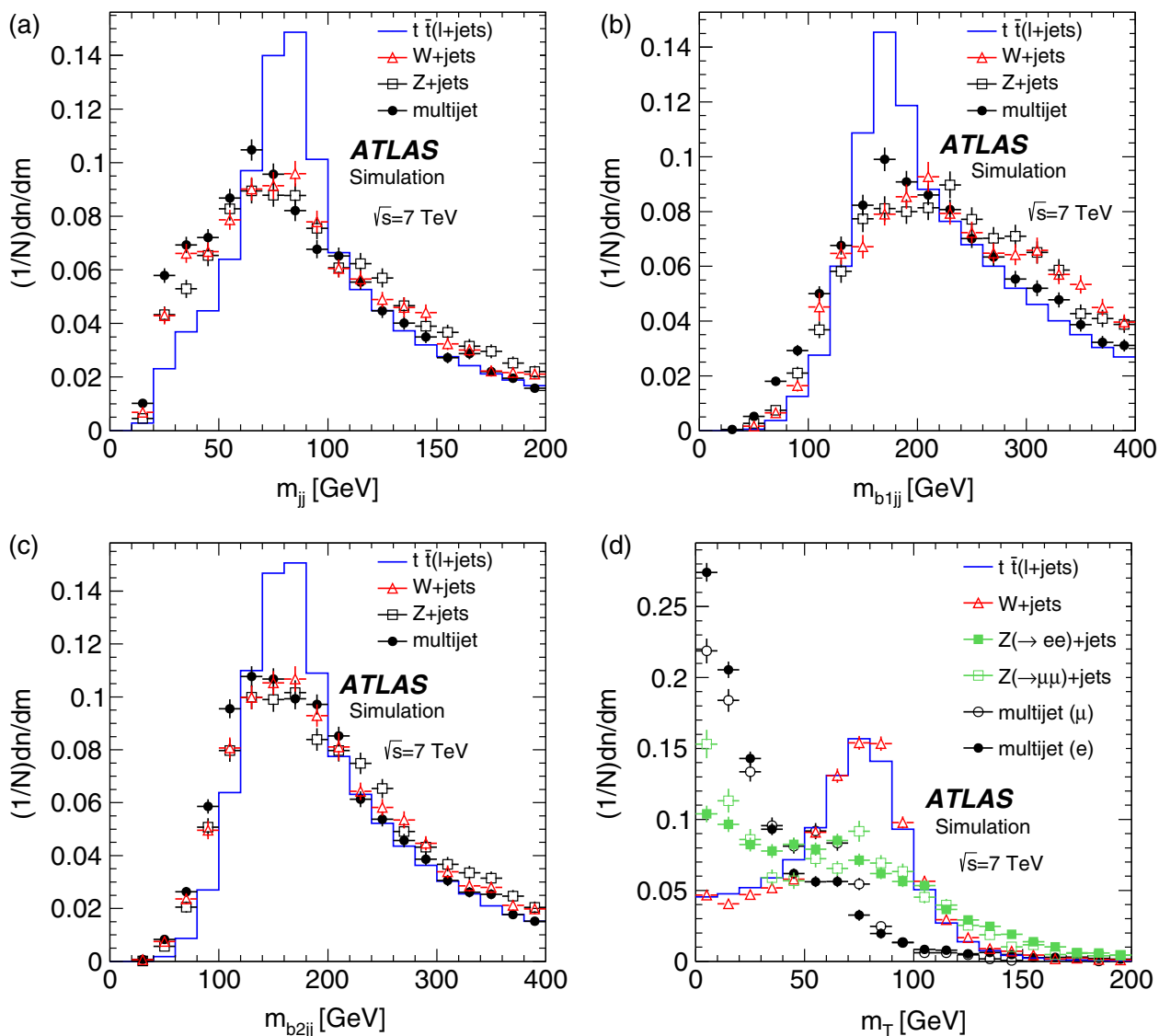

FIG. 1 (color online). (a) Invariant mass of two highest- $p_{\mathrm{T}}$ jets not designated as $b$-jets $\left(m_{j j}\right)$, (b) and (c) invariant masses of jets designated as $b$-jets and the jets used for $m_{j j},\left(m_{b 1 j j}\right)$ and $\left(m_{b 2 j j}\right)$, where $b 1$ stands for the leading $b$-jet and $b 2$ for the subleading $b$-jet, and (d) transverse mass of lepton and $E_{\mathrm{T}}^{\mathrm{miss}}\left(m_{\mathrm{T}}\right)$. The distributions have been normalized and show distributions for $t \bar{t} \rightarrow \ell+$ jets, $Z(\rightarrow \ell \ell)+$ jets, $W(\rightarrow \ell \nu)+$ jets MC events and multijet events populating the $\ell+$ jets channels. The $e$ and $\mu$ channels have been merged together in the $m_{j j}, m_{b 1 j j}$ and $m_{b 2 j j}$ distributions. They are kept separate in the $m_{\mathrm{T}}$ distributions except for $t \bar{t}$ and $W+$ jets. Events are required to have exactly one isolated $e$ or $\mu, E_{\mathrm{T}}^{\text {miss }}>30 \mathrm{GeV}$, at least four jets, and at least one $b$-tagged jet.

quite distinct for $t \bar{t} \rightarrow \ell+$ jets while there is very little difference between the various backgrounds. The distributions for $t \bar{t} \rightarrow \ell+$ jets events show that they include top quarks decaying to $b+W$ with the $W$ boson decaying to jets. On the other hand, the $m_{\mathrm{T}}$ distributions show that they include a $W$ boson decaying leptonically in both the $t \bar{t} \rightarrow$ $\ell+$ jets and $W+$ jets channels but cannot discriminate between them. They do show a clear separation between final states with one $W$ boson decaying leptonically and those with little intrinsic $E_{\mathrm{T}}^{\text {miss }}(Z+$ jets and multijets).

The background templates for $Z+$ jets events from MC simulation are checked with $Z+$ jets events from data by selecting events with two identified leptons and requiring the dilepton mass to be near the $Z$ mass. Events are required to have two oppositely charged leptons $\left(p_{\mathrm{T}}^{e}>25 \mathrm{GeV}\right.$ and $\left.p_{\mathrm{T}}^{\mu}>20 \mathrm{GeV}\right), 70 \mathrm{GeV}<m_{\ell \ell}<110 \mathrm{GeV}, E_{\mathrm{T}}^{\text {miss }}>30 \mathrm{GeV}$, and the same jet selections as for the $\ell+$ jets signal. The only significant background in the control data sample is from the $t \bar{t} \rightarrow \ell \ell^{\prime}+$ jets channel. Figure 2 shows the $m_{j j}$, $m_{b 1 j j}$ and $m_{b 2 j j}$ distributions after merging $e e$ and $\mu \mu$ events for ALPGEN $Z+$ jets MC simulation and the data after applying scale factors (SF) based on comparing data and simulation as a function of the $Z$ boson $p_{\mathrm{T}}$ and the jet multiplicity. The small expected $t \bar{t}$ contribution is subtracted from the data distributions. The KolmogorovSmirnov goodness-of-fit test (KS) value in each plot indicates how well the shape of the data distribution is described by the ALPGEN MC simulation. ${ }^{4}$ Since there is no noticeable difference between the shapes of the $W+$ jets and $Z+$ jets templates, as shown in Fig. 1, one can conclude that both $\mathrm{MC}$ templates can reproduce reasonably well the distributions expected in the data. The number of selected $Z+$ jets events is also predicted well by the simulation.

${ }^{4} \mathrm{KS}$ is calculated with the function supplied by ROOT for comparing the compatibility of two histograms [44]. 

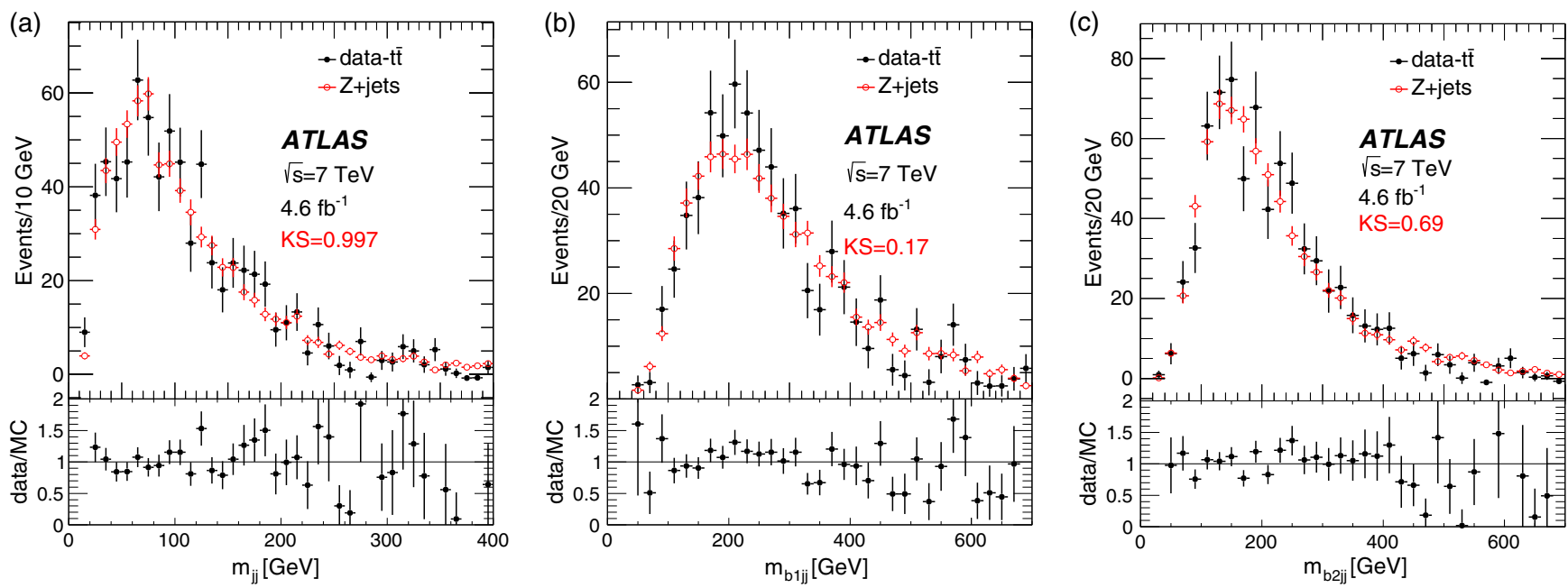

FIG. 2 (color online). (a) Invariant mass of two highest- $p_{\mathrm{T}}$ jets not designated as $b$-jets $\left(m_{j j}\right)$, (b) and (c) invariant masses of jets designated as $b$-jets and the jets used for $m_{j j},\left(m_{b 1 j j}\right)$ and $\left(m_{b 2 j j}\right)$, where $b 1$ stands for the leading $b$-jet and $b 2$ for the subleading $b$-jet, and (d) transverse mass of lepton and $E_{\mathrm{T}}^{\text {miss }}\left(m_{\mathrm{T}}\right)$. The distributions show ALPGEN MC for a control sample of $Z(\rightarrow \ell \ell)+$ jets events selected by requiring $70<m_{\ell \ell}<110 \mathrm{GeV}$ ( $m_{\ell \ell}$ is the invariant mass of the two leptons), $E_{\mathrm{T}}^{\text {miss }}>30 \mathrm{GeV}$, at least four jets, and at least one of them $b$-tagged, compared to the data after subtracting the expected $t \bar{t}$ contribution. KS is the value of the KolmogorovSmirnov goodness-of-fit test.

\section{B. Fits to mass distributions}

As shown in Sec. VIA the three invariant masses constructed from jets do not discriminate between the various backgrounds, while the signal from $t \bar{t}$ is quite distinct. The only distribution that is different for each background is the transverse mass. In particular, the transverse mass clearly distinguishes final states with intrinsic $E_{\mathrm{T}}^{\text {miss }}$, i.e. those with a $W$ boson decaying to a lepton and neutrino, from those where $E_{\mathrm{T}}^{\text {miss }}$ is due to mismeasurements. The dominant processes without sizeable intrinsic $E_{\mathrm{T}}^{\text {miss }}$ are multijet and $Z+$ jets. The transverse mass distributions for those two processes are different. However, they contribute little in $m_{\mathrm{T}}>40 \mathrm{GeV}$ so most of the separation comes from the region below $40 \mathrm{GeV}$. As shown in Fig. 2, the ALPGEN $Z+$ jets simulation predicts the shape and the number of $Z+$ jets events well, so the choice is made to normalize the number of $Z+$ jets events to that predicted by the simulation. The number of single top events is similarly normalized from MC simulation. The amount of multijet background is obtained from the fit to the data using the templates derived from nonisolated lepton samples. The other free parameters are the total number of $W+$ jets events and the total number of $t \bar{t}$ events. The fractional contributions for the various $t \bar{t}$ channels are obtained using MC events. To ensure that events are not used more than once, two sets of data are fitted: $E_{\mathrm{T}}^{\text {miss }}<30 \mathrm{GeV}$ (set 1) and $E_{\mathrm{T}}^{\text {miss }}>30 \mathrm{GeV}$ (set 2). Set 1 is used to fit the $m_{\mathrm{T}}$ distributions and helps determine the multijet background. Set 2 is used to fit the three jet mass distributions. Both sets are fit simultaneously with three parameters: the total number of multijet events, the total number of $W+$ jets events and the total number of $t \bar{t}$ events.

The variables $m_{b 1 j j}$ and $m_{b 2 j j}$ are strongly correlated with $m_{j j}$. To exploit the fact that the correlations are very different in $t \bar{t}$ and the background, the fits are done simultaneously in $6 \times 6 \times 6$ bins of $m_{j j}, m_{b 1 j j}$ and $m_{b 2 j j}$ for a total of 216 bins. Of those, 30 bins have zero events since they are kinematically not possible. The ranges and bin sizes are chosen so that all bins used for fitting are populated by more than 10 events. That limits the range of $m_{\mathrm{T}}$ to $m_{\mathrm{T}}<120 \mathrm{GeV}, m_{j j}$ to $m_{j j}<250 \mathrm{GeV}, m_{b 1 j j}$ to $m_{b 1 j j}<450 \mathrm{GeV}$, and $m_{b 2 j j}$ to $m_{b 2 j j}<450 \mathrm{GeV}$.

The $m_{\mathrm{T}}$ distributions for events with $E_{\mathrm{T}}^{\text {miss }}<30 \mathrm{GeV}$, used in the fits, are shown in Fig. 3. Table I shows the predicted contributions from each channel, combining events with $E_{\mathrm{T}}^{\text {miss }}<30 \mathrm{GeV}$ and $E_{\mathrm{T}}^{\text {miss }}>30 \mathrm{GeV}$. Figure 4 shows that the fits describe well the full $e+$ jets and $\mu+$ jets event distributions of $m_{j j}, m_{b 1 j j}$ and $m_{b 2 j j}$ after requiring $E_{\mathrm{T}}^{\text {miss }}>30 \mathrm{GeV}$. Figure 5 shows the $m_{\mathrm{T}}$ distribution for events with $E_{\mathrm{T}}^{\text {miss }}>30 \mathrm{GeV}$ compared with the predicted contributions, which agree well with the data.

Noticeable features from these fits are as follows:

(i) The largest backgrounds originate in $W+$ jets $(15 \%)$ and other $t \bar{t}$ channels (8.5\%); the rest add up to $12 \%$ (multijets 5.3\%, $Z+$ jets 3.9\%, and single top 3.0\%).

(ii) The numbers of $t \bar{t}$ and $W+$ jets events obtained by fitting are in good agreement with those predicted by the SM. 

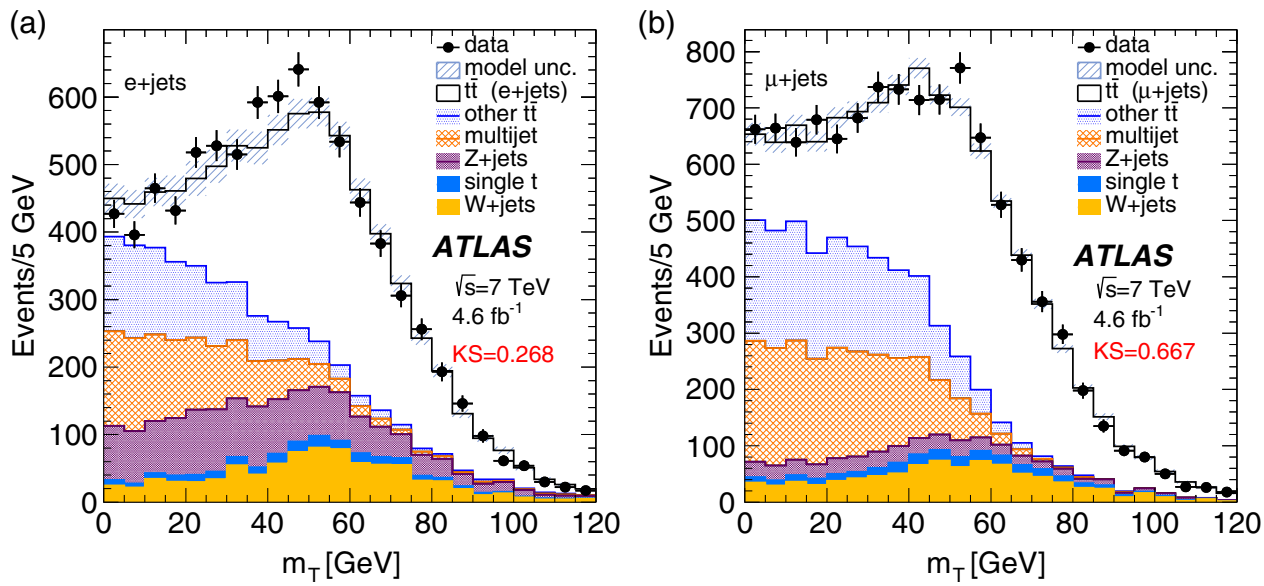

FIG. 3 (color online). Transverse mass of lepton and $E_{\mathrm{T}}^{\mathrm{miss}}\left(m_{\mathrm{T}}\right)$ distributions used in the fits. Events are required to have exactly one isolated $e$ or $\mu, E_{\mathrm{T}}^{\text {miss }}<30 \mathrm{GeV}$, at least four jets, and at least one $b$-tag. The model uncertainty (model unc.) is the sum in quadrature of the statistical uncertainties of the templates used in the fits. KS is the value of the Kolmogorov-Smirnov goodness-of-fit test. (a) $e+$ jets, (b) $\mu+$ jets.

\section{DILEPTON + JETS CHANNEL}

The number of $t \bar{t} \rightarrow \ell \ell^{\prime}+$ jets events in the data is extracted by fitting two dilepton invariant mass distributions: one with $30<E_{\mathrm{T}}^{\text {miss }}<60 \mathrm{GeV}$ and the other with $E_{\mathrm{T}}^{\text {miss }}>60 \mathrm{GeV}$. The most significant background to the

TABLE I. Results from fitting $e+$ jets and $\mu+$ jets mass distributions from $\ell+$ jets events requiring exactly one isolated lepton $(e$ or $\mu)$, at least four jets, and at least one $b$-tag. The uncertainties quoted here are from the fits and do not include systematic uncertainties. The $Z+$ jets contribution is normalized to the MC expectation. In addition to MC statistical uncertainty, it includes the uncertainty from the scale factors applied to the simulation in order to match the jet multiplicity and the $\mathrm{Z}$ boson $p_{\mathrm{T}}$ dependence to that observed in the data. The single top and diboson contributions are normalized to $\mathrm{MC}$ predictions, include only MC statistical uncertainty and the SM cross section uncertainty. The (MC) rows give the numbers expected from MC simulation. The $\chi^{2} /$ ndf row gives the $\chi^{2}$ and degrees of freedom of the fits.

\begin{tabular}{lcc}
\hline \hline Channel & $e+$ jets & $\mu+$ jets \\
\hline$t \bar{t} \rightarrow \ell+$ jets & $19710 \pm 280$ & $25090 \pm 310$ \\
$(\mathrm{MC})$ & $(18966 \pm 31)$ & $(24233 \pm 34)$ \\
$t \bar{t}($ other $)$ & $2674 \pm 30$ & $3393 \pm 30$ \\
$(\mathrm{MC})$ & $(2577 \pm 11)$ & $(3277 \pm 16)$ \\
$W+$ jets & $4800 \pm 500$ & $5600 \pm 500$ \\
$(\mathrm{MC})$ & $(4140 \pm 70)$ & $(5850 \pm 90)$ \\
$Z+$ jets (MC) & $1900 \pm 500$ & $790 \pm 200$ \\
Single top (MC) & $910 \pm 70$ & $1170 \pm 80$ \\
Diboson (MC) & $5.0 \pm 0.2$ & $6.1 \pm 0.2$ \\
Multijet & $1000 \pm 120$ & $2800 \pm 140$ \\
Total background & $11333 \pm 700$ & $13700 \pm 600$ \\
Signal + background & $31000 \pm 800$ & $38800 \pm 700$ \\
Data & 30733 & 40414 \\
$\chi^{2} /$ ndf & $188 / 207$ & $218 / 207$ \\
\hline \hline
\end{tabular}

$t \bar{t} \rightarrow \ell \ell^{\prime}+$ jets channels after requiring $E_{\mathrm{T}}^{\text {miss }}>30 \mathrm{GeV}$ and at least one $b$-tagged jet comes from the $Z\left(\rightarrow \ell \ell^{\prime}\right)+$ jets, with a smaller contribution from single top production (4\%). Since the $E_{\mathrm{T}}^{\text {miss }}$ distribution falls more rapidly for the $Z+$ jets background than for the $t \bar{t}$ signal process separating it into two $E_{\mathrm{T}}^{\mathrm{miss}}$ bins improves the sensitivity of the fit for separating the two processes. Backgrounds from dibosons and jets misidentified as isolated leptons (mainly from $\mathrm{W}+$ jets with leptons from heavy-quark semileptonic decays or an isolated charged hadron misidentified as a lepton, together denoted as nonprompt leptons) amount to $1.0 \%$ of the events. The background from nonprompt isolated leptons is estimated from the number of data events with lepton pairs with the same charge after subtracting a very small expected contribution from diboson processes. The invariant mass distributions are fitted with three templates: one derived from a $t \bar{t} \mathrm{MC}$ sample, one from a $Z+$ jets MC sample, and one summed over all other contributions. Only the amounts contributed by $t \bar{t}$ and $Z+$ jets are allowed to vary. The $Z$ boson background in the $e \mu+$ jets channel from the $Z(\rightarrow \tau \tau \rightarrow e \mu)+\mathrm{X}$ channel is too small to be extracted by a fit, so $m_{e \mu}$ is fitted only for the number of $t \bar{t}$ events in the data while the background is fixed. The fits in the $\ell \ell$ channel are performed over a mass range from $40 \mathrm{GeV}$ to $250 \mathrm{GeV}$ and in the $e \mu$ channel over a mass range from $10 \mathrm{GeV}$ to $250 \mathrm{GeV}$. Figures 6 and 7 show that the $m_{\ell \ell^{\prime}}$ and $E_{\mathrm{T}}^{\text {miss }}$ distributions are well described in all dilepton channels. Results of the fits are given in Table II.

\section{LEPTON $+\tau_{\text {had }}+$ JETS CHANNEL}

Unlike the single-lepton + jets and dilepton channels the background in the $\ell \tau_{\text {had }}+$ jets channel is not small and is dominated by contributions from other $t \bar{t}$ channels. Thus, invariant masses and other kinematic variables are not 

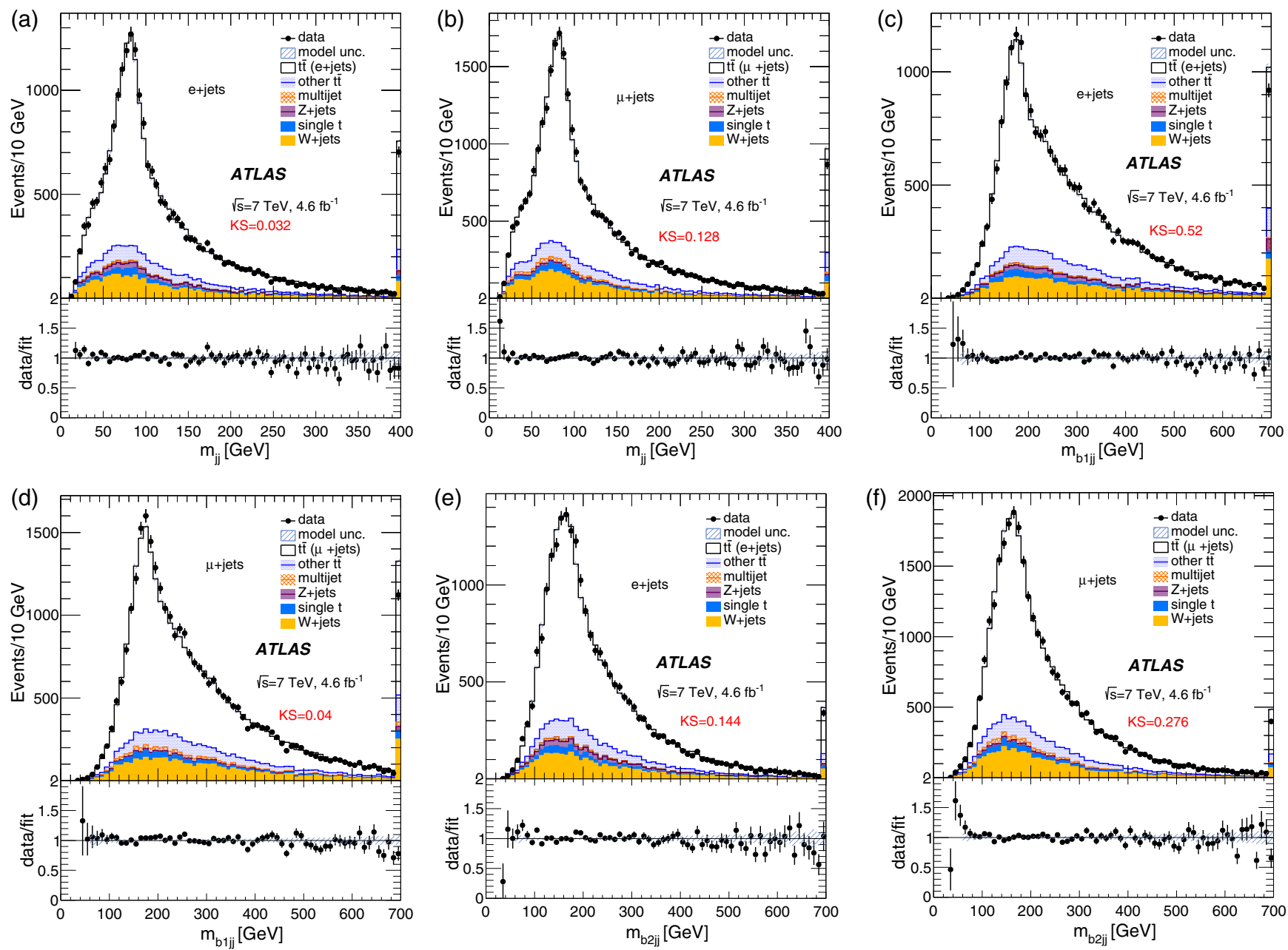

FIG. 4 (color online). Distributions in data compared to the SM expectations after fitting the following distributions: (a),(b) the invariant mass of two highest- $p_{\mathrm{T}}$ jets not designated as $b$-jets; (c),(d) the invariant mass of the leading jet designated as $b$-jet and the jets used for $m_{j j}\left(m_{b 1 j j}\right)$, and (e),(f) the invariant mass of the second jet designated as a $b$-jet and the two jets used for $m_{j j}\left(m_{b 2 j j}\right)$. The distributions are shown for events with isolated leptons, at least four jets, at least one $b$-tag, and $E_{\mathrm{T}}^{\text {miss }}>30 \mathrm{GeV}$, with the $e+$ jets and $\mu+$ jets channels separated. The last bin shows the overflow. The ratio plots show the result of dividing the data points by the model expectation. The model uncertainty (model unc.) is the sum in quadrature of the statistical uncertainties of the templates used in the fits. $\mathrm{KS}$ is the value of the Kolmogorov-Smirnov goodness-of-fit test.

sufficiently sensitive to separate signal and background. In this case a BDT multivariate discriminant, named $\mathrm{BDT}_{j}$, is used to separate $\tau$ leptons from jets identified as $\tau$ candidates (see Sec. V). Compared to the previous ATLAS measurement with this channel [15], the present analysis uses only one-prong $\tau$ decays and is based on a larger data sample with a different background model to reduce the statistical uncertainty on the background prediction.

\section{A. Tau background templates}

In order to separate the contribution of processes with $\tau$ leptons (signal) from those with jets misidentified as $\tau$ (fake $\tau$ ) the $\mathrm{BDT}_{j}$ distributions of selected events are fitted with templates for fake $\tau$ distributions derived from data and true $\tau$ lepton distributions derived from MC simulation. Control data samples to obtain templates of jets misidentified as $\tau$ candidates are selected with the following requirements:

(i) exactly one isolated electron with $p_{\mathrm{T}}^{e}>25 \mathrm{GeV}$ and no identified muons for the $e+\tau$ channel;

(ii) or exactly one isolated muon with $p_{\mathrm{T}}^{\mu}>20 \mathrm{GeV}$ and no identified electrons for the $\mu+\tau$ channel;

(iii) and no additional muons with $p_{\mathrm{T}}>4 \mathrm{GeV}$;

(iv) and $40 \mathrm{GeV}<m_{\mathrm{T}}\left(\ell, E_{\mathrm{T}}^{\text {miss }}\right)<100 \mathrm{GeV}$;

(v) and exactly one $\tau$ candidate and at most one additional jet.

There are two mutually exclusive control samples:

The $W+1$-jet sample contains a lepton, one jet misidentified as a $\tau$ candidate and no additional jets. The $W+2$-jets sample contains a lepton and exactly two jets with the lower $p_{\mathrm{T}}$ jet misidentified as a $\tau$ candidate. 

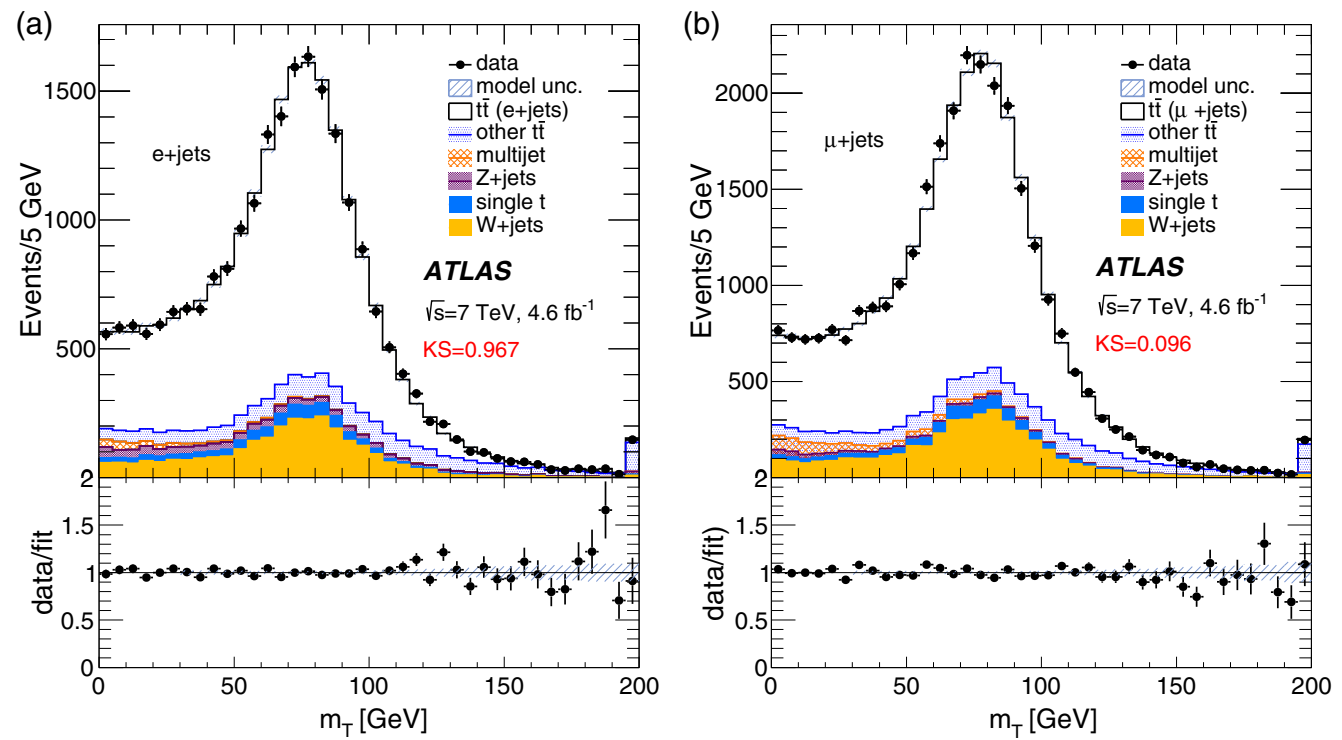

FIG. 5 (color online). The transverse mass of lepton and $E_{\mathrm{T}}^{\mathrm{miss}}\left(m_{\mathrm{T}}\right)$ distributions for events with isolated leptons, at least four jets, at least one $b$-tag and $E_{\mathrm{T}}^{\text {miss }}>30 \mathrm{GeV}$ in the $e+$ jets and $\mu+$ jets channels. The last bin shows the overflow. The ratio plots show the result of dividing the data points by the model expectation. The model uncertainty (model unc.) is the sum in quadrature of the statistical uncertainties of the templates used in the fits. KS is the value of the Kolmogorov-Smirnov goodness-of-fit test. (a) $e+$ jets, (b) $\mu+$ jets.

The control samples are divided into two subsamples, one with $\tau$ and $\ell$ having the opposite-sign charges (OS), and the other with $\tau$ and $\ell$ having the same-sign charges (SS). The $W+1$-jet sample is rich in jets originating from quark hadronization (quark jets) while the $W+2$-jets sample has a high percentage of jets originating from gluon hadronization (gluon jets) as determined from MC studies. One can extract the distributions of gluon jets misidentified as $\tau$ candidates since the number of gluon jets in OS and SS samples must be the same because they are not correlated with the charge of the lepton. Fake $\tau$ template shapes depend on the jet type. Those from light-quark jets peak at higher $\mathrm{BDT}_{j}$ values than those from gluon jets. The signal contributes only to OS events. Therefore, the $\mathrm{BDT}_{j}$ distributions of OS events are fitted with a pair of background templates, whose linear combination equals the sum of the OS light-quark and gluon jets identified as $\tau$ candidates, and a signal $\tau$ template. MC studies show that requiring $\tau$ candidates that have only one associated charged particle strongly suppresses jets originating from heavy quarks ( $c$-jets, $b$-jets). The $b$-jets are further suppressed by excluding $\tau$ candidates that are tagged as $b$-jets. The $\mathrm{BDT}_{j}$ template from remaining $c$-jets identified as $\tau$ candidates is similar to the light-quark template. The signal template is constructed by summing the expected contribution of any channel that has a real $\tau$ lepton or a lepton misidentified as a $\tau$ lepton.

In the $W+2$-jets sample the lower- $p_{\mathrm{T}}$ jet has a high probability of coming from final- or initial-state radiation and thus a high probability of being a gluon jet. In the following, OS1 (SS1) stands for the $\tau$ fake $\mathrm{BDT}_{j}$ distribution obtained from OS (SS) $W+1$-jet data sets and OS2 (SS2) represent the equivalent distribution for $W+2$-jets. Figures 8(a) and 8(b) show the OS and OS-SS distributions normalized to compare the shapes. It can be seen that there are significant differences between OS1 and OS2, but if one subtracts the SS distribution from the OS distribution (OS-SS) the shapes are in good agreement. The distributions are a sum of light-quark jets and gluon jets, and can be described by the following equations:

$$
\begin{aligned}
& \mathrm{OS} 1=a_{1} \cdot \mathrm{OS}_{\mathrm{q}}+b_{1} \cdot G, \\
& \mathrm{SS} 1=c_{1} \cdot \mathrm{SS}_{\mathrm{q}}+b_{1} \cdot G, \\
& \mathrm{OS} 2=a_{2} \cdot \mathrm{OS}_{\mathrm{q}}+b_{2} \cdot G, \\
& \mathrm{SS} 2=c_{2} \cdot \mathrm{SS}_{\mathrm{q}}+b_{2} \cdot G,
\end{aligned}
$$

where $\mathrm{OS}_{\mathrm{q}}\left(\mathrm{SS}_{\mathrm{q}}\right)$ is a function describing the shape of the distribution of light-quark jets contributing to OS (SS) and $G$ is the corresponding function for gluon jets. The observation that the OS1-SS1 and OS2-SS2 distributions have the same shape leads to the conclusion that $a_{1} / c_{1}=$ $a_{2} / c_{2}$ for any $E_{\mathrm{T}}$ as the $E_{\mathrm{T}}$ of $\tau$ candidates from $W+2$-jets are significantly lower than those from $W+1$-jet. Using the above equations, one can extract the $G$ function from the OS and SS distributions separately, i.e.

$$
\begin{gathered}
K \cdot G=(R \cdot \mathrm{OS} 2-\mathrm{OS} 1), \\
K \cdot G=(R \cdot \mathrm{SS} 2-\mathrm{SS} 1),
\end{gathered}
$$



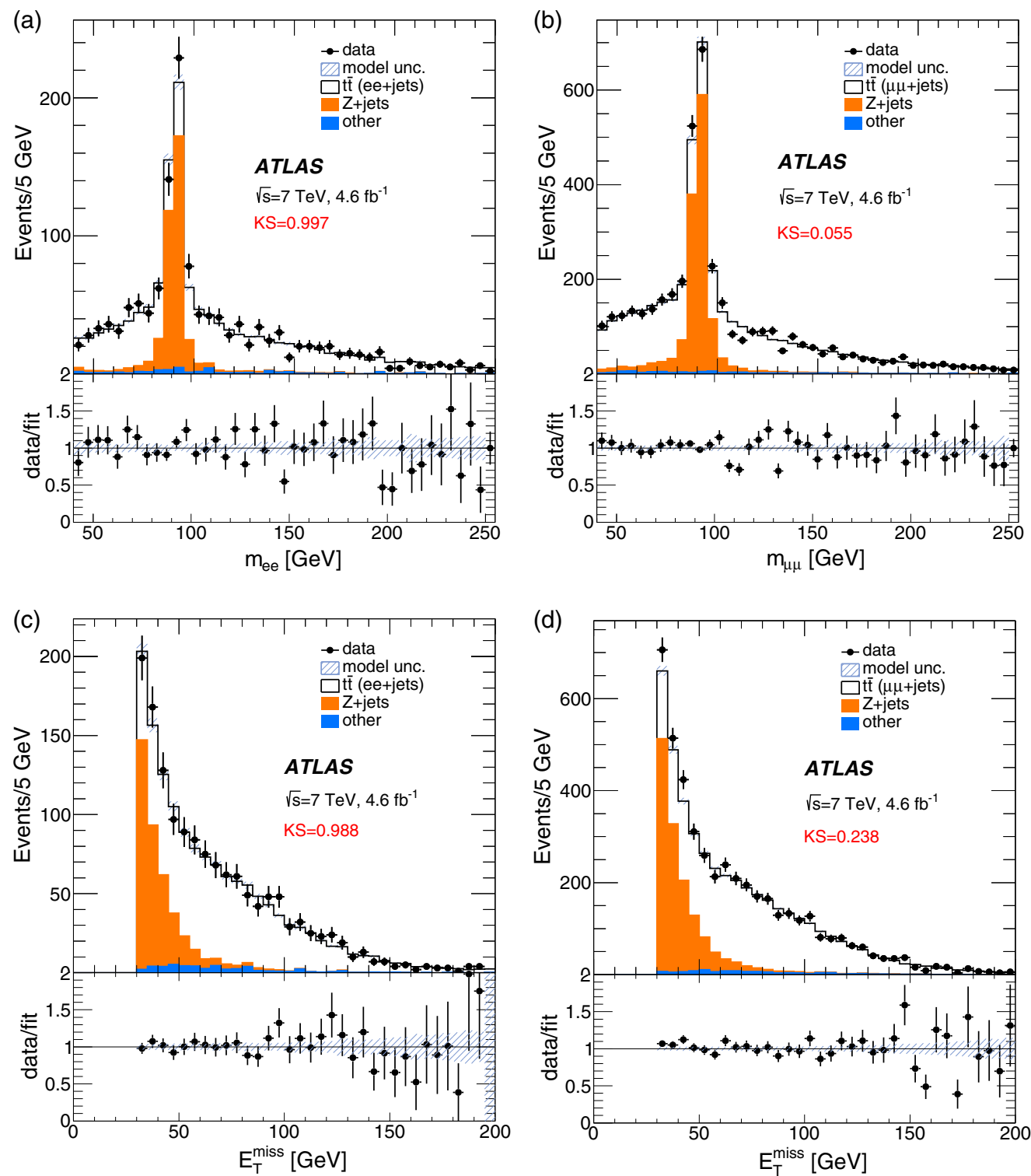

FIG. 6 (color online). Dilepton invariant masses (a) $m_{e e}$, (b) $m_{\mu \mu}$, and $E_{\mathrm{T}}^{\mathrm{miss}}$ distributions for events with two isolated leptons, $E_{\mathrm{T}}^{\text {miss }}>30 \mathrm{GeV}$, at least two jets, and at least one $b$-tag in the (c) $e e+$ jets and (d) $\mu \mu+$ jets channels. The $Z+$ jet entries include a small contribution from $Z \rightarrow \tau^{+} \tau^{-}$with both $\tau$ leptons decaying to $e$ or $\mu$. The ratio plots show the result of dividing the data points by the fit. The model uncertainty (model unc.) is the sum in quadrature of the statistical uncertainties of the templates used in the fits. KS is the value of the Kolmogorov-Smirnov goodness-of-fit test.

where $R$ is the ratio of the total number of OS1-SS1 events to OS2-SS2 events and $K=R \cdot b_{2}-b_{1}$ is an unknown constant that must be the same whether SS or OS is used to extract $G$. Figure $8(\mathrm{c})$ shows the extracted $K \cdot G$ distributions for $\tau$ candidates. It is seen that the OS and SS distributions are fully consistent with each other and can be summed to reduce the statistical uncertainties.

In principle any background $\mathrm{BDT}_{j}$ distribution can be described by a linear combination of $G$ and OS1 distributions. Furthermore, the $\mathrm{BDT}_{j}$ distributions depend on $E_{\mathrm{T}}$ of the $\tau$ candidates, which differs from sample to sample. The $E_{\mathrm{T}}$ dependence of the $\mathrm{BDT}_{j}$ is taken into account by fitting separate $E_{\mathrm{T}}$ regions with templates derived for those regions weighted to reproduce the $E_{\mathrm{T}}$ distributions of the expected background. The OS1 sample has a small (2\%) number of $\tau$ leptons from dibosons and $Z \rightarrow \tau^{+} \tau^{-}$final states that have no impact on the fits to $\ell \tau_{\text {had }}+$ jets $\mathrm{BDT}_{j}$ data distributions whether or not they are subtracted from the OS1 template.

\section{B. Signal extraction by fitting to $\mathrm{BDT}_{j}$ shape}

The final background normalization and signal measurement are established through fitting templates to the data. There are various classes of background: 

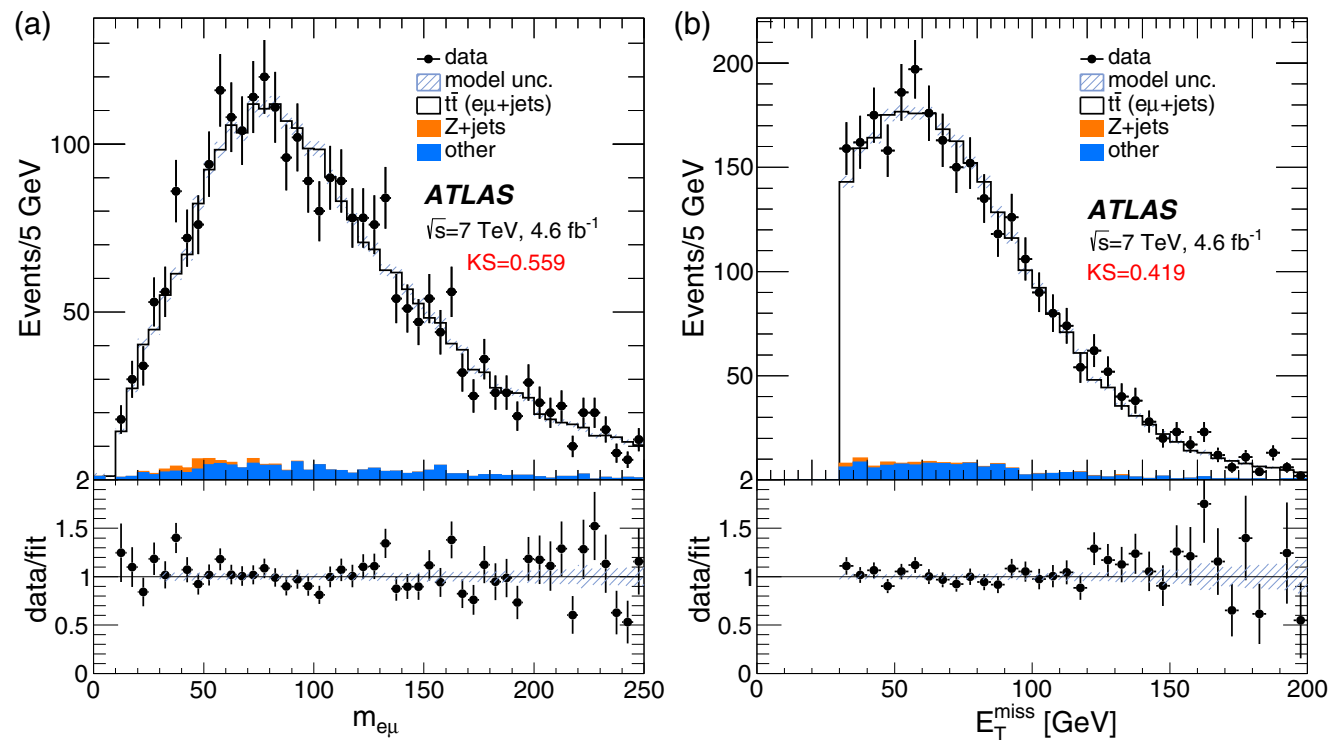

FIG. 7 (color online). (a) Invariant mass of electron and muon $\left(m_{e \mu}\right)$ and (b) $E_{\mathrm{T}}^{\text {miss }}$ distributions for $e \mu$ events after requiring one isolated $e$ and one isolated $\mu, E_{\mathrm{T}}^{\text {miss }}>30 \mathrm{GeV}$, at least two jets, and at least one $b$-tag. The ratio plots show the result of dividing the data points by the fit. The model uncertainty (model unc.) is the sum in quadrature of the statistical uncertainties of the templates used in the fits. KS is the value of the Kolmogorov-Smirnov goodness-of-fit test.

(1) from processes with an isolated $\ell$ where a jet is misidentified as a $\tau$ candidate;

(2) from processes other than $t \bar{t}$ that have $\tau$ leptons and an isolated $\ell$;

(3) from processes with two isolated $\ell$ where one $\ell$ is misidentified as a $\tau$ candidate;

(4) from multijet processes where both $\ell$ and $\tau$ are from one jet misidentified as an isolated $\ell$ and another as a $\tau$ candidate.

TABLE II. Results from fitting $\ell \ell^{\prime}$ invariant mass distributions using two $E_{\mathrm{T}}^{\text {miss }}$ regions from $\ell \ell^{\prime}+$ jets events requiring two isolated leptons ( $e$ or $\mu$ ), $E_{\mathrm{T}}^{\text {miss }}>30 \mathrm{GeV}$, at least two jets, and at least one $b$-tag. The numbers of events are after summing events from both $E_{\mathrm{T}}^{\text {miss }}$ regions $E_{\mathrm{T}}^{\text {miss }}<60 \mathrm{GeV}$ and $E_{\mathrm{T}}^{\text {miss }}>60 \mathrm{GeV}$. The uncertainties are from the fits and do not include systematic uncertainties. The single top and diboson contributions are normalized to the SM predictions and include only the MC statistical uncertainty and the uncertainty on the SM cross section. The (MC) rows give the numbers expected from MC simulation.

\begin{tabular}{lccc}
\hline \hline Channel & $\mu \mu+$ jets & $e e+$ jets & $e \mu+$ jets \\
\hline$t \bar{t}$ & $2890 \pm 80$ & $1000 \pm 40$ & $2640 \pm 50$ \\
$(\mathrm{MC})$ & $(2536 \pm 11)$ & $(903 \pm 6)$ & $(2420 \pm 11)$ \\
$Z+$ jets & $1380 \pm 50$ & $379 \pm 11$ & $13 \pm 4$ \\
$(\mathrm{MC})$ & $(1267 \pm 8)$ & $(385 \pm 11)$ & $(13 \pm 4)$ \\
Single top (MC) & $86 \pm 8$ & $36 \pm 7$ & $98 \pm 9$ \\
Diboson (MC) & $22 \pm 1$ & $8.1 \pm 0.5$ & $3.3 \pm 0.3$ \\
Fake leptons & $17 \pm 10$ & $17 \pm 8$ & $19 \pm 10$ \\
Total background & $1430 \pm 50$ & $442 \pm 15$ & $136 \pm 12$ \\
Signal + background & $4400 \pm 100$ & $1440 \pm 40$ & $2770 \pm 80$ \\
Data & 4102 & 1447 & 2848 \\
$\chi^{2} /$ ndf & $35 / 34$ & $31 / 34$ & $58 / 49$ \\
\hline \hline
\end{tabular}

The dominant background to the $t \bar{t} \rightarrow \ell \tau_{\text {had }}+$ jets channel comes from the $t \bar{t} \rightarrow \ell+$ jets channel with one jet misidentified as a $\tau$ candidate (class 1 ). The only powerful suppression technique for that background is $\tau$ identification, thus the best variable is the $\mathrm{BDT}_{j}$ score, described with the $\tau$ candidate selection in Sec. V. Background of classes 1 and 4 is taken into account using templates consisting of light-quark jet $\tau$ fakes and gluon jet $\tau$ fakes derived from enriched $W+$ jets data samples as described in Sec. VIII A.

The signal $\mathrm{BDT}_{j}$ template is derived from MC $\tau$ candidates that are matched to a $\tau$ lepton or a lepton from MC events that satisfy the event selection (classes 2 and 3). The class 2 processes contributing to the signal template are $t \bar{t} \rightarrow \ell \tau+$ jets, $Z\left(\rightarrow \tau^{+} \tau^{-}\right)+$jets, and small contributions from single top and diboson events. The main backgrounds of class 3 are $Z \rightarrow e^{+} e^{-}$ and $t \bar{t}$ events. Most electrons are removed by the $\mathrm{BDT}_{e}$ cut (see Sec. V); the few that remain are indistinguishable from $\tau$ leptons. There is an even smaller number of muons overlapping with $\tau$ candidates that are not removed by the muon veto and are also indistinguishable from $\tau$ leptons. In these cases, the $\tau$ candidates are added to the signal template. The efficiency for electrons and muons misidentified as $\tau$ candidates is determined by studying $Z \rightarrow \ell^{+} \ell^{-}$events. Based on these studies the estimated contribution from class 3 background to the signal template is $2.8 \%$. The total contribution from class 2 and class 3 backgrounds ( $Z+$ jets, $t \bar{t} \rightarrow \ell \ell+$ jets, single top and dibosons) to the signal template is $15 \%$. Table III shows the detailed composition of the signal templates. 

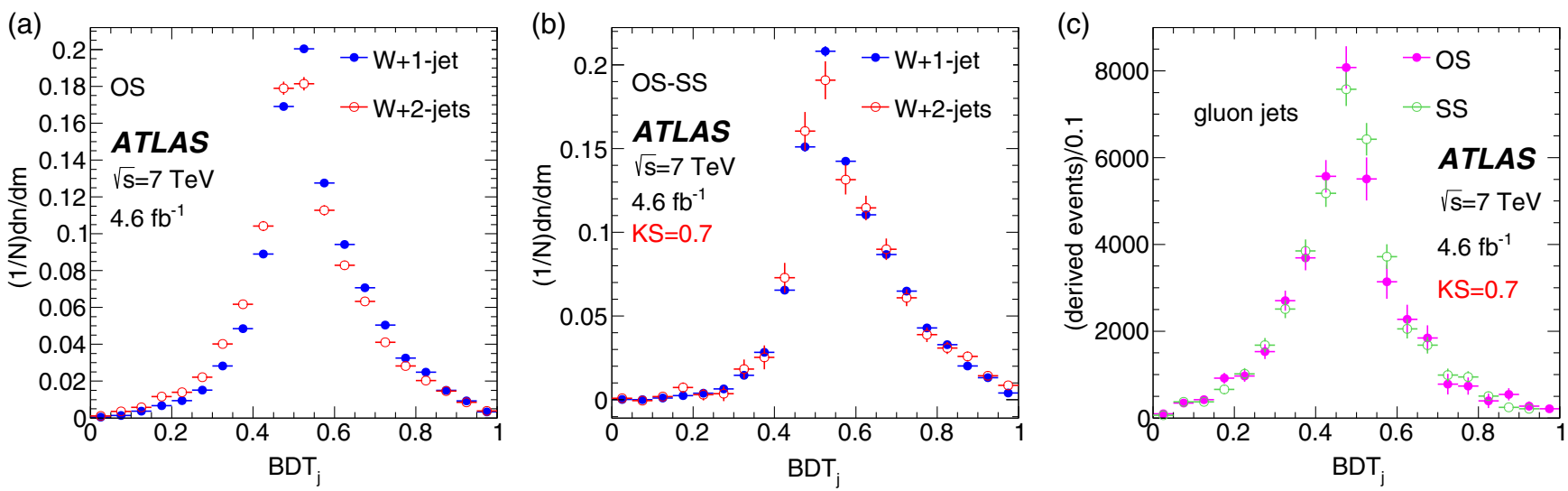

FIG. 8 (color online). Normalized distributions of the output of the boosted decision tree used to discriminate $\tau$ leptons from jets misidentified as $\tau \mathrm{s}, \mathrm{BDT}_{j}$, for $\tau$ candidates from $W+1$-jet and $W+2$-jets samples for leptons with opposite sign (OS), the distribution

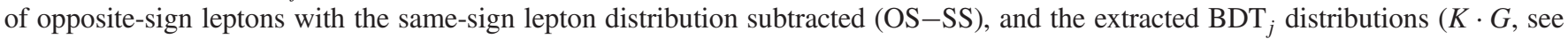
text) for gluon jets misidentified as $\tau$ candidates is shown. (a) OS, (b) OS-SS, (c) gluons jets.

With these background templates and MC signal template $(S)$, a $\chi^{2}$ fit is performed with parameters to set the normalization of each template: $a \cdot \mathrm{OS} 1+b \cdot G+c \cdot S$. The combined $e$ and $\mu$ channel results are obtained by fitting to the sum of the distributions. Comparisons of the template shapes of the $e$ and $\mu$ channel show they are identical within the uncertainties.

Two different $E_{\mathrm{T}}$ regions, $20 \mathrm{GeV} \leq E_{\mathrm{T}}^{\tau} \leq 35 \mathrm{GeV}$ and $35 \mathrm{GeV} \leq E_{\mathrm{T}}^{\tau} \leq 100 \mathrm{GeV}$, are chosen such that each region has the same number of expected signal events. Three parameters are used to fit both regions simultaneously: the fraction of $\tau$ candidates in each $E_{\mathrm{T}}$ region that are gluon jets and the total fraction of signal. In the fit the sum of signal and background must add up to the number of observed events in each $E_{\mathrm{T}}$ region and the amount of signal in the two regions is constrained by the ratio predicted from MC simulation.

\section{Fit results}

The three-parameter fit was applied to MC samples to establish whether it can extract the known signal without

TABLE III. Composition of signal template: all events from MC simulation with a true $\tau, e$ or $\mu$ matched to the $\tau$ candidate. The number of events are normalized to the number expected from simulation. Regions 1 and 2 are $20 \mathrm{GeV} \leq E_{\mathrm{T}}^{\tau} \leq 35 \mathrm{GeV}$ and $35 \mathrm{GeV} \leq E_{\mathrm{T}}^{\tau} \leq 100 \mathrm{GeV}$, respectively. The uncertainties represent the statistical uncertainties of the MC samples.

\begin{tabular}{lcc}
\hline \hline Channel & Region 1 & Region 2 \\
\hline$t \bar{t} \rightarrow \ell \tau_{\text {had }}+$ jets & $611.5 \pm 5.4$ & $621.4 \pm 5.4$ \\
$t \bar{t} \rightarrow \ell \ell+$ jets & $13.0 \pm 0.7$ & $13.0 \pm 0.7$ \\
$Z+$ jets & $54.5 \pm 3.3$ & $45.3 \pm 3.0$ \\
Single top & $23.6 \pm 2.3$ & $27.1 \pm 2.4$ \\
Dibosons & $1.5 \pm 0.2$ & $2.2 \pm 0.3$ \\
Total & $705.2 \pm 6.8$ & $709.5 \pm 6.8$ \\
\hline \hline
\end{tabular}

bias. The MC samples are made with events from $t \bar{t}$, $W+$ jets, $Z+$ jets, single top and diboson final states satisfying the data selection criteria. The MC samples were split into two, one used as the data to fit and the other to generate the templates for the fit. Figure 9 shows these MC fit results after correcting the background templates derived from $W+$ jets to account for the different $E_{\mathrm{T}}$ distribution of the $\tau$ candidates in the expected background to $t \bar{t} \rightarrow \ell \tau_{\text {had }}+$ jets. The model uncertainty shown in Fig. 9 corresponds to the uncertainty of the templates in the fits to the data and used for ensemble tests. The ensemble tests show that no bias is introduced by the fitting procedure. The $\mu$ and $e$ channels are combined by adding together the distributions of both channels. The data $\mathrm{BDT}_{j}$ distributions can have multiple entries for an event as all $\tau$ candidates are considered. This has no impact on the $t \bar{t} \rightarrow \ell \tau_{\text {had }}+$ jets signal as there is only one $\tau$ lepton decaying to hadrons in that channel.

The results of fitting the data are summarized in Table IV. $N_{S}^{\text {Fitted }}$ is the number of signal template events. $N_{t \bar{t}}^{\mathrm{Fitted}}$ is the number of observed $t \bar{t} \rightarrow \ell \tau+$ jets events, obtained by subtracting the contributions from class 2 and class 3 backgrounds (see Sec. VIII B) from $N_{S}^{\text {Fitted }}$. The number of expected $\left(N_{\bar{t} \bar{t}}^{\mathrm{MC}}\right)$ is in good agreement with $N_{t \bar{t}}^{\mathrm{Fitted}}$. Figure 10 shows the final results using these $\mu$ and $e$ channel combined templates.

Jets misidentified as $\tau$ leptons come mostly from $t \bar{t} \rightarrow$ $\ell+$ jets and from $W+$ jets. Thus the $m_{\mathrm{T}}$ distributions should show a Jacobian peak from a $W$ decay. The $t \bar{t} \rightarrow$ $\ell \tau_{\text {had }}+$ jets events have additional neutrinos, which produce a broader $m_{\mathrm{T}}$ distribution. Figure 11 shows the distributions from events selected with $\mathrm{BDT}_{j}<0.6$, which are mostly background, and for events selected with $\mathrm{BDT}_{j}>0.7$ where the ratio of signal to all background is $2: 1$. The plots include the predicted distributions using the normalizations based on the fits to the $\mathrm{BDT}_{j}$ 

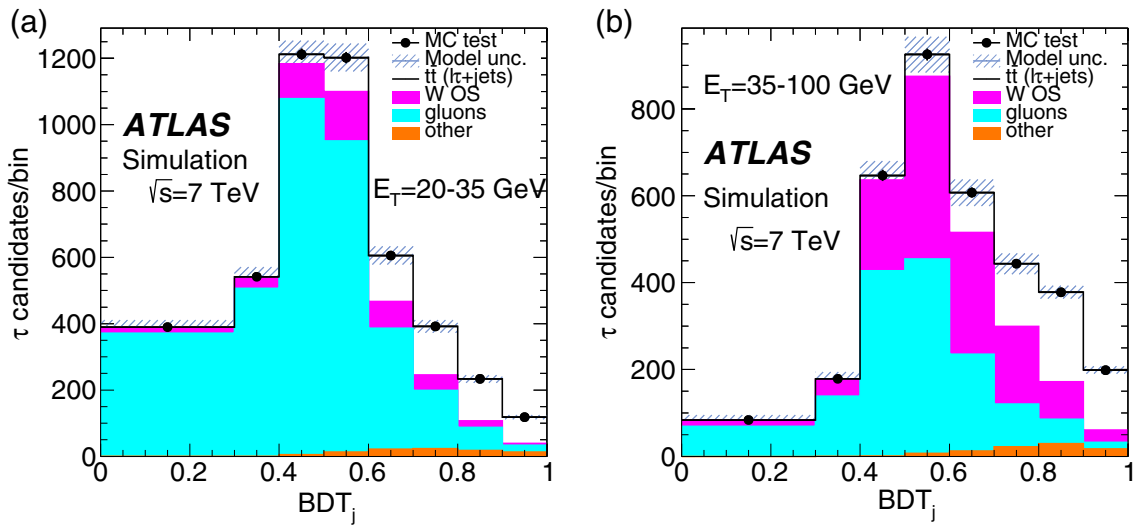

FIG. 9 (color online). Fitted distributions of the $\tau$-jet discriminant BDT $\mathrm{MC}_{j}$ using corrected background templates for two $E_{T}$ regions. The model uncertainty is the uncertainty of the templates used in the fits to the data. (a) $E_{T}=20-35 \mathrm{GeV}$, (b) $E_{T}=35-100 \mathrm{GeV}$.

distributions. The amount of $Z \rightarrow \tau \tau$ is normalized to the MC prediction. The data are well reproduced in all cases.

\section{MEASURING CROSS SECTION AND BRANCHING RATIOS}

In the SM $100 \%$ of the top quark decays have one $W$ boson and a quark. Therefore the top quark branching ratios into channels with leptons and jets are determined by the $W$ decay branching ratios that have been measured with $0.3 \%$ precision (assuming lepton universality) [13] and are predicted by the SM with an uncertainty of order $0.1 \%$. It is possible to derive the branching ratios into all decay modes using the number of $t \bar{t}$ events extracted in the previous sections assuming that the top quark branching ratios to leptons and jets add up to $100 \%$. Any deviation from the $W$ branching ratios would be an indication of some process not predicted by the SM. The following observed quantities are defined (where $\mathcal{A}_{\mathrm{ch}} \cdot \epsilon_{\mathrm{ch}}$ is the geometric detector acceptance times the efficiency of channel $c h$ ):

(i) $N_{\mu j}=($ observed number of $t \bar{t} \rightarrow \mu+$ jets $) / \mathcal{A}_{\mu j} \cdot \epsilon_{\mu j}$,

(ii) $N_{e j}=$ (observed number of $t \bar{t} \rightarrow e+$ jets) $/ \mathcal{A}_{e j} \cdot \epsilon_{e j}$,

(iii) $N_{\mu \mu}=$ (observed number of $t \bar{t} \rightarrow \mu+\mu+$ jets) $/ \mathcal{A}_{\mu \mu}$. $\epsilon_{\mu \mu}$,

(iv) $N_{e e}=($ observed number of $t \bar{t} \rightarrow e+e+$ jets $) / \mathcal{A}_{e e}$. $\epsilon_{e e}$,

(v) $N_{e \mu}=$ (observed number of $t \bar{t} \rightarrow e+\mu+$ jets $) / \mathcal{A}_{e \mu}$. $\epsilon_{e \mu}$, (vi) $N_{\ell \tau}=$ (observed number of $t \bar{t} \rightarrow \ell+\tau_{\text {had }}+$ jets $) /$ $\mathcal{A}_{\ell \tau} \cdot \epsilon_{\ell \tau}$,

(vii) $N_{\ell j}=N_{\mu j}+N_{e j}$,

(viii) $N_{\ell \ell}=N_{\mu \mu}+N_{e e}+N_{e \mu}$.

The following notation is used for the top quark branching ratios:

(i) $B_{\mu}$ : top quark branching ratio to $\mu \nu_{\mu}\left(\nu_{\tau}\right)+X$,

(ii) $B_{e}$ : top quark branching ratio to $e \nu_{e}\left(\nu_{\tau}\right)+X$,

(iii) $B_{\tau}$ : top quark branching ratio to $\tau \nu_{\tau}+X$, with the $\tau$ lepton decaying hadronically,

(iv) $B_{j}$ : top quark branching ratio to jets,

(v) $B_{\ell}: B_{\mu}+B_{e}$

The branching ratios $B_{\mu}$ and $B_{e}$ include events with leptonic $\tau$ decays.

With these definitions the following relations hold:

$$
\begin{gathered}
N_{\ell j}=2 \sigma_{t \bar{t}} \cdot B_{\ell} \cdot B_{j} \cdot \mathcal{L}, \\
N_{\ell \ell}=\sigma_{t \bar{t}} \cdot B_{\ell}^{2} \cdot \mathcal{L}, \\
N_{\ell \tau}=2 \sigma_{t \bar{t}} \cdot B_{\ell} \cdot B_{\tau} \cdot \mathcal{L}, \\
B_{j}+B_{\ell}+B_{\tau}=1,
\end{gathered}
$$

where $\sigma_{t \bar{t}}$ is the cross section for $t \bar{t}$ pair production and $\mathcal{L}$ is the integrated luminosity. These four equations with four unknowns can be solved to obtain:

TABLE IV. Numbers of events expected from MC simulation and fit results to the $\mathrm{BDT}_{j}$ distribution using background and signal templates as described in Sec. VIII A. $N_{t \overline{\mathrm{MC}}}^{\mathrm{MC}}$ is the expected number of $t \bar{t} \rightarrow \ell \tau_{\text {had }}+$ jets events for a cross section of $177 \mathrm{pb}$. $\mathrm{B}_{\mathrm{non}} \bar{t} \tau$ is the number of $\tau$ leptons expected from sources other than $t \bar{t} \rightarrow \ell \tau_{\text {had }}+$ jets. $\mathrm{B}_{\text {lepton }}$ is the expected number of leptons misidentified as $\tau$ leptons. $N_{S}^{\text {Fitted }}$ is the number of events extracted with the signal template $\left(S\right.$, see text) and $N_{\bar{t}}^{\text {Fitted }}=N_{S}^{\text {Fitted }}-\mathrm{B}_{\text {non } t \bar{\tau} \tau}-\mathrm{B}_{\text {lepton }}$

\begin{tabular}{lccccc}
\hline \hline & $N_{t \bar{t}}^{\mathrm{MC}}$ & $\mathrm{B}_{\text {non } t \bar{t} \tau}$ & $\mathrm{B}_{\text {lepton }}$ & $N_{S}^{\text {Fitted }}$ & $N_{t \bar{t}}^{\text {Fitted }}$ \\
\hline $20<E_{\mathrm{T}}^{\tau}<35 \mathrm{GeV}$ & $611 \pm 5$ & $76.2 \pm 3.5$ & $17.1 \pm 1.1$ & N/A & N/A \\
$35<E_{\mathrm{T}}^{\tau}<100 \mathrm{GeV}$ & $621 \pm 5$ & $69.5 \pm 3.3$ & $17.6 \pm 1.1$ & N/A & N/A \\
Combined $E_{\mathrm{T}}^{\tau}$ bins & $1232 \pm 8$ & $146 \pm 5$ & $34.8 \pm 1.5$ & $1460 \pm 60\left(\chi^{2} / \mathrm{ndf}=0.69\right)$ & $1280 \pm 60$ \\
\hline \hline
\end{tabular}



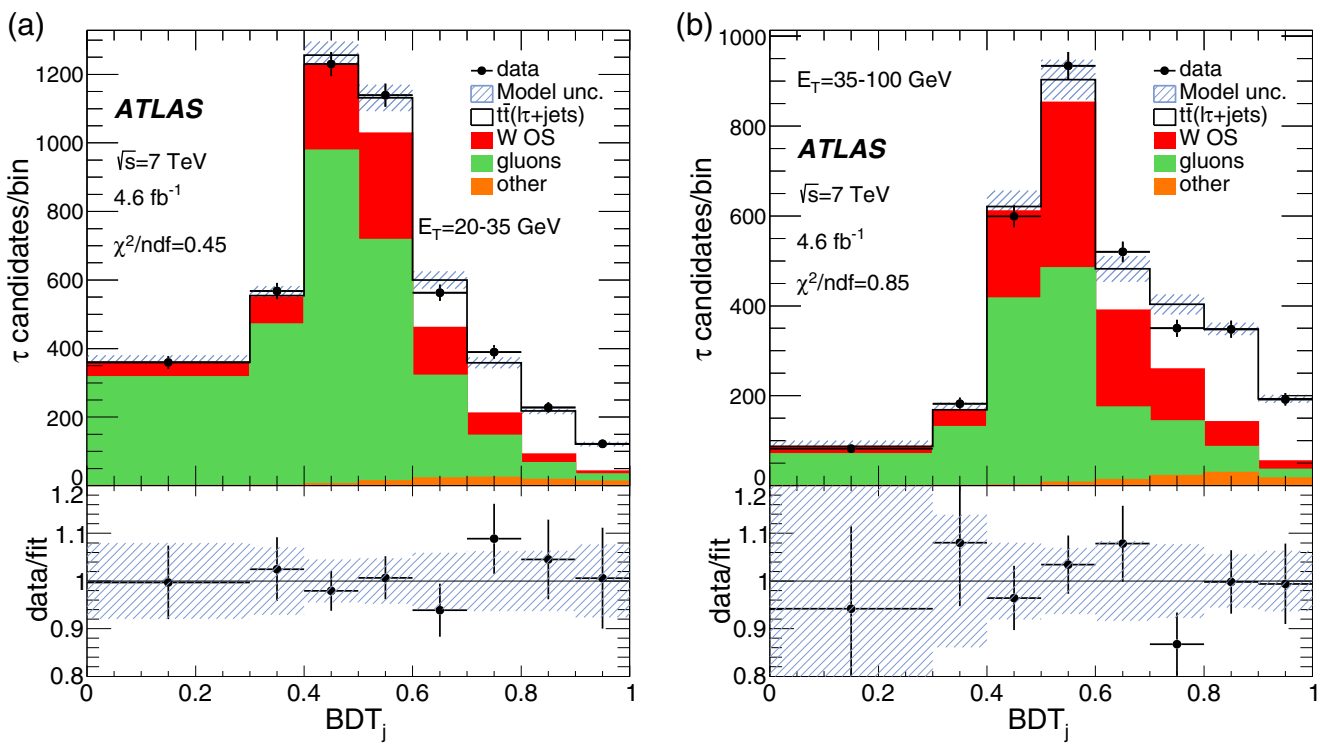

FIG. 10 (color online). Fitted distributions of the $\tau$-jet discriminant BDT $_{j}$ in data using corrected background templates for (a) $20 \mathrm{GeV} \leq E_{\mathrm{T}} \leq 35 \mathrm{GeV}$ and (b) $35 \mathrm{GeV} \leq E_{\mathrm{T}} \leq 100 \mathrm{GeV}$. The model uncertainty is the statistical uncertainty of the templates used in the fits. (a) $E_{T}=20-35 \mathrm{GeV}$, (b) $E_{T}=35-100 \mathrm{GeV}$.

$$
\begin{gathered}
B_{j}=N_{\ell j} /\left(N_{\ell j}+2 N_{\ell \ell}+N_{\ell \tau}\right), \\
B_{\ell}=2 N_{\ell \ell} /\left(N_{\ell j}+2 N_{\ell \ell}+N_{\ell \tau}\right), \\
B_{\tau}=N_{\ell \tau} /\left(N_{\ell j}+2 N_{\ell \ell}+N_{\ell \tau}\right), \\
\sigma_{t \bar{t}} \cdot \mathcal{L}=\left(N_{\ell j}+2 N_{\ell \ell}+N_{\ell \tau}\right)^{2} / 4 N_{\ell \ell} .
\end{gathered}
$$

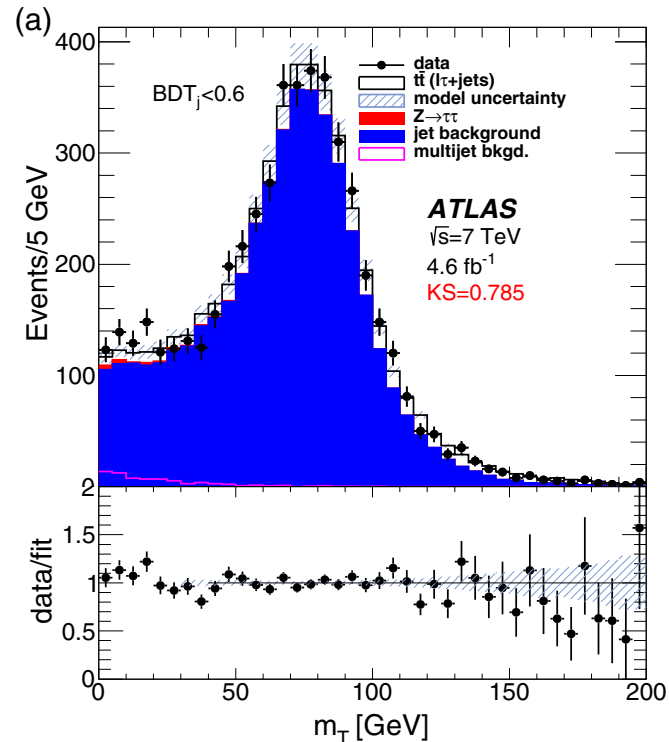

From the numbers of $t \bar{t}$ events given in Tables I-IV and the acceptances given in Table $\mathrm{V}$ the values are obtained for $N_{\ell x}$ and given in Table VI. The $N_{\ell x}$ are in units of events $/ \mathrm{pb}^{-1}$.

After solving for $B_{\ell}$ one can solve for $B_{e}$ and $B_{\mu}$ using ratios in the dilepton and the single-lepton channel:

$$
B_{\mu(e)}=2 N_{\mu \mu(e e)} \cdot B_{j} / N_{\mu(e) j} \equiv a,
$$

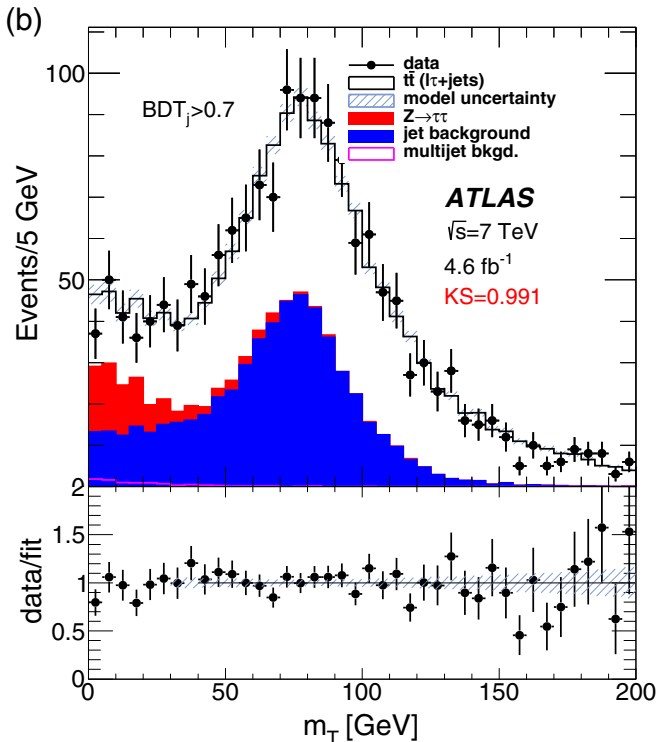

FIG. 11 (color online). Transverse mass distributions $\left(m_{\mathrm{T}}\right)$ of $t \bar{t} \rightarrow \ell \tau_{\text {had }}+$ jets events. The black points are data, the solid histograms the prediction based on the fits to the $\mathrm{BDT}_{j}$ distributions. The jet background is the sum of all channels with jets misidentified as $\tau$ candidates normalized to the amount obtained from the fits to $\mathrm{BDT}_{j}$ distributions. The multijet background is the estimated contribution from non- $t \bar{t}$ multijet processes and is included in the jet background. The model uncertainty is the statistical uncertainty of the templates used in the fits. KS is the value of the Kolmogorov-Smirnov goodness-of-fit test. (a) $\mathrm{BDT}_{j}<0.6$, (b) $\mathrm{BDT}_{j}<0.7$. 
TABLE V. The acceptance $\times$ efficiency $\left(\mathcal{A}_{\mathrm{ch}} \cdot \epsilon_{\mathrm{ch}}\right)$ of each channel used to extract the number of $t \bar{t}$ events after all selections. The $\mathcal{A}_{\mathrm{ch}} \cdot \epsilon_{\mathrm{ch}}$ are calculated by taking the ratio of fully reconstructed MC events to MC generated events. The uncertainties represent the statistical uncertainties of the MC samples.

\begin{tabular}{ccccccc}
\hline \hline & $e+$ jets & $\mu+$ jets & $e e+$ jets & $\mu \mu+$ jets & $e \mu+$ jets & $\ell \tau+$ jets \\
\hline $\mathcal{A}_{\mathrm{ch}} \cdot \epsilon_{\mathrm{ch}}(\%)$ & $14.02 \pm 0.02$ & $17.88 \pm 0.02$ & $7.09 \pm 0.04$ & $19.74 \pm 0.08$ & $9.50 \pm 0.04$ & $4.36 \pm 0.02$ \\
\hline \hline
\end{tabular}

TABLE VI. Measured number of events $/ \mathrm{pb}^{-1}$ for each channel and the number predicted by the SM. Data uncertainties are statistical only. The SM uncertainty is calculated using the theoretical uncertainty of the NNLO + NNLL calculation of the cross section.

\begin{tabular}{|c|c|c|c|c|c|c|}
\hline & $N_{e j}$ & $N_{\mu j}$ & $N_{e e}$ & $N_{\mu \mu}$ & $N_{e \mu}$ & $N_{\ell \tau}$ \\
\hline & \multicolumn{2}{|c|}{$N_{\ell j}$} & \multicolumn{3}{|c|}{$N_{\ell \ell}$} & \\
\hline Measured & $\begin{array}{r}30.62 \pm 0.26 \\
61\end{array}$ & $\begin{array}{l}30.57 \pm 0.29 \\
.40\end{array}$ & $3.06 \pm 0.12$ & $\begin{array}{c}3.19 \pm 0.10 \\
12.31 \pm 0.20\end{array}$ & $6.06 \pm 0.12$ & $6.39 \pm 0.30$ \\
\hline SM & $30.40 \pm 1.2$ & $\begin{array}{l}30.40 \pm 1.2 \\
2.4\end{array}$ & $2.86 \pm 0.11$ & $\begin{array}{c}2.86 \pm 0.11 \\
10.95 \pm 0.44\end{array}$ & $5.72 \pm 0.20$ & $6.39 \pm 0.25$ \\
\hline
\end{tabular}

$$
B_{\mu(e)}=B_{\ell} \cdot \sqrt{N_{\mu \mu(e e)} / N_{\ell \ell}} \equiv b .
$$

The best values are obtained by minimizing

$$
\chi^{2}=\left(\left[B_{\mu(e)}-a\right] / \delta a\right)^{2}+\left(\left[B_{\mu(e)}-b\right] / \delta b\right)^{2},
$$

where $\delta a$ and $\delta b$ are the $a$ and $b$ uncertainties.

\section{SYSTEMATIC UNCERTAINTIES}

Several sources of experimental and theoretical systematic uncertainty are considered. Lepton trigger, reconstruction and selection efficiencies are assessed in data and $\mathrm{MC}$ simulation by comparing the $Z \rightarrow \ell^{+} \ell^{-}$ events selected with the same object criteria as used for the $t \bar{t}$ analyses. Scale factors are applied to MC samples when calculating acceptances to account for any differences between predicted and observed efficiencies. The scale factors are evaluated by comparing the observed efficiencies with those determined with simulated $Z$ boson events. Systematic uncertainties on these scale factors are evaluated by varying the selection of events used in the efficiency measurements and by checking the stability of the measurements over the course of data taking. The modeling of the lepton momentum scale and resolution is studied with reconstructed invariant mass distributions of $Z \rightarrow \ell^{+} \ell^{-}$candidate events, and these distributions are used to adjust the simulation accordingly [36,37].

The jet energy scale (JES), jet energy resolution (JER), and their uncertainties are derived by combining information from test-beam data, LHC collision data and simulation. For jets within the acceptance, the JES uncertainty varies in the range $4 \%-8 \%$ as a function of jet $p_{\mathrm{T}}$ and $\eta$ [39]. The $b$-tagging efficiency and its uncertainty is determined using a sample of jets containing muons [40]. The effect of all these variations on the final result is evaluated by varying each source of systematic uncertainty by $\pm 1 \sigma$ in the MC-derived templates and fitting all the distributions with the new templates.

The uncertainty in the kinematic distributions of the $t \bar{t}$ signal events gives rise to systematic uncertainties in the signal acceptance, with contributions from the choice of generator, the modeling of initial- and final-state radiation (ISR/FSR) and the choice of PDF set. The generator uncertainty is evaluated by comparing the MC@NLO and ALPGEN [29] predictions with those of POWHEG [20] interfaced to either HERWIG or PYTHIA. The PDF uncertainty is evaluated following the PDF4LHC recommendation [45]. An event-by-event weighting is applied to a default MC@NLO sample that uses the central value of CT10 [28]. MSTW2008 [46] and NNPDF2.0 [47,48] sets are taken to estimate the systematic uncertainty due to the PDF. The uncertainty due to ISR/FSR is evaluated using the ALPGEN generator interfaced to the PYTHIA shower model, and by varying the parameters controlling ISR and FSR in a range consistent with experimental data [49]. The dominant uncertainty in this category of systematic uncertainties is the modeling of ISR/FSR. In addition there is an uncertainty in the $W+$ jets MC simulation due to the uncertainty in the heavy-flavor component of the jets. The systematic uncertainty from single top $\mathrm{MC}$ simulation has a negligible impact on the overall systematic uncertainty.

The $\tau$ identification uncertainty is derived from a template fit to the $\mathrm{BDT}_{j}$ distribution from an enriched $Z \rightarrow$ $\tau^{+} \tau^{-}$data sample selected with the same $\mu$ and $\tau$ candidate requirements as the sample for this analysis, but with fewer than two jets and $m_{\mathrm{T}}<20 \mathrm{GeV}$ to remove $W+$ jets events. The background templates are the $W+1$-jet OS and the gluon template used in the fit to the $t \bar{t}$ data sample. The signal template is the $\mathrm{BDT}_{j}$ distribution from $Z \rightarrow \tau^{+} \tau^{-}$ 
TABLE VII. Absolute systematic uncertainties, in pb, for the cross-section measurements with the $t \bar{t} \rightarrow \ell \tau_{\text {had }}+$ jets channel. The $e$ and $\mu$ uncertainties are the sum in quadrature of trigger, reconstruction and selection efficiency uncertainties. The $\tau$ identification uncertainty includes electrons misidentified as $\tau$ leptons.

\begin{tabular}{lc}
\hline \hline & Absolute uncertainties [pb] \\
\hline$\mu$ uncertainty & 1.7 \\
$e$ uncertainty & 3.0 \\
Jet energy scale & $-5.5 /+6.8$ \\
Jet energy resolution & 1.5 \\
ISR/FSR & 12.3 \\
MC generator & 10.1 \\
PDF & 0.6 \\
$b$-tag & $-8.3 /+10.0$ \\
$\tau$ identification & 8.0 \\
$\tau$ background correction & 5.6 \\
Total & $-22 /+23$ \\
Luminosity & 3.3 \\
\hline \hline
\end{tabular}

MC events. The uncertainty includes the statistical uncertainty of the data samples, the uncertainty in the $Z$ inclusive cross section measured by ATLAS [50] (excluding luminosity uncertainty) and jet energy scale uncertainty. The signal template shape uncertainty, estimated from fits to the $Z \rightarrow \tau^{+} \tau^{-}$data sample, is found to be negligible. The uncertainty on the number of misidentified electrons $(<0.5 \%)$, determined from an enriched $Z \rightarrow e^{+} e^{-}$data sample, is included. In addition there is an uncertainty in the correction applied to the $\tau$ background templates derived from $W+$ jets data to account for the different $E_{\mathrm{T}}$ distribution of the $\tau$ candidates in the expected background to $t \bar{t} \rightarrow \ell \tau_{\text {had }}+$ jets.
The calculated systematic uncertainties for the inclusive cross section measured with the $\ell \tau_{\text {had }}+$ jet channel are given in Table VII. Table VIII gives the systematic uncertainties estimated when combining all channels. The uncertainty on the measured integrated luminosity is estimated to be $1.8 \%$ [51]. As expected the systematic uncertainties are substantially larger in the measurement of the cross section based on the $\ell \tau_{\text {had }}+$ jets channel alone than in the combination of all channels. The largest uncertainty in the combined cross-section measurement and in the branching ratio measurements is due to the JES uncertainty, followed by the MC generator and the uncertainty in the heavy-flavor component of $W+$ jets. The uncertainties on the measured branching ratios are significantly smaller than on the measured inclusive cross section, as expected due to cancellations. $B_{\tau}$ has a larger systematic uncertainty than the other branching ratios due to uncertainties on $\tau$ identification that do not cancel in the ratios.

\section{RESULTS}

The inclusive $t \bar{t}$ cross section using only the $\ell \tau_{\text {had }}+$ jets channel is derived from the number of observed $t \bar{t} \rightarrow$ $\ell \tau+$ jets events given in Table IV (Sec. VIII C):

$$
\sigma_{t \bar{t}}=183 \pm 9 \text { (stat.) } \pm 23 \text { (syst.) } \pm 3 \text { (lumi.) pb. }
$$

This result is consistent with the previous ATLAS measurement, $186 \pm 25 \mathrm{pb}$ [15]. This measurement differs from the earlier one in that it uses only $\tau \mathrm{s}$ decaying into one charged hadron and a different background model to reduce the systematic uncertainties in the branching ratios. The results from combining all channels to extract the top quark branching ratios are given in Table IX. The measured cross

TABLE VIII. Relative systematic uncertainties (\%) for cross section and branching ratio measurements. The systematic uncertainties for $B_{e}$ and $B_{\mu}$ (not shown) are $100 \%$ correlated with the $B_{\ell}$ uncertainties and of the same size. The $e$ and $\mu$ uncertainties are the sum in quadrature of trigger, reconstruction and selection efficiency uncertainties. The MC generator uncertainty is the difference between POWHEG interfaced with PYTHIA and ALPGEN interfaced with HERWIG. HF stands for heavy flavor.

\begin{tabular}{lcccc}
\hline \hline & $\sigma_{t \bar{t}}$ & $B_{j}$ & $B_{\ell}$ & $B_{\tau}$ \\
\hline$\mu$ uncertainty & 1.3 & 0.15 & 0.6 & 0.5 \\
$e$ uncertainty & 1.1 & 0.15 & 0.5 & 0.5 \\
Jet energy scale & $-6.9 /+4.9$ & $-1.6 /+1.4$ & $-1.9 /+2.7$ & $-3.8 /+4.3$ \\
Jet energy resolution & 1.2 & 0.3 & 0.8 & 0.7 \\
ISR/FSR & 2.0 & 0.3 & 1.3 & 4.0 \\
MC generator & 3.6 & 0.6 & 0.8 & 1.9 \\
PDF & 2.9 & 0.3 & 0.1 & 0.3 \\
$b$-tag & $-1.3 /+5.0$ & 0.3 & 1.0 & 1.5 \\
$\tau$ identification & 0.5 & 0.15 & 1.1 & 3.5 \\
$\tau$ background correction & 0.2 & $<0.1$ & $-1.1 /+2.3$ & 2.5 \\
$W+$ jets HF content & $-4.1 /+2.7$ & $-1.0 /+0.7$ & $-3.4 /+4.2$ & $-1.3 /+2.1$ \\
Total & $-9.7 /+9.2$ & $-2.1 /+1.8$ & $<0.1$ & $-7.1 /+7.6$ \\
Luminosity & 1.8 & $<0.1$ & $<0.1$ \\
\hline \hline
\end{tabular}


TABLE IX. Measured cross section (pb) and top quark branching ratios (\%) including statistical and systematic uncertainties without imposing lepton universality. The top quark branching ratios add up to $100.2 \%$ because of rounding precision. The uncertainty on the SM prediction for the cross section is the uncertainty in the NNLO + NNLL theoretical calculation [24,55]. The SM branching ratios are the predicted $W$ branching ratios. The LEP measurements represent the $W$ branching ratios obtained by combining results for ALEPH, DELPHI, L3 and OPAL collaborations imposing lepton universality [13]. The LEP entries $B_{e}$ and $B_{\mu}$ include the $\tau$ leptonic decays that have been subtracted from $B_{\tau}$.

\begin{tabular}{lcrr}
\hline \hline & Measured (top quark) & SM & LEP $(W)$ \\
\hline$\sigma_{t \bar{t}}$ & $178 \pm 3$ (stat.) \pm 16 (stat.) \pm 3 (lumi.) pb & $177.3 \pm 9.0_{-6.0}^{+4.6} \mathrm{pb}$ & \\
\hline$B_{j}$ & $66.5 \pm 0.4$ (stat.) \pm 1.3 (syst.) & $67.51 \pm 0.07$ & $67.48 \pm 0.28$ \\
$B_{e}$ & $13.3 \pm 0.4$ (stat.) \pm 0.5 (syst.) & $12.72 \pm 0.01$ & $12.70 \pm 0.20$ \\
$B_{\mu}$ & $13.4 \pm 0.3$ (stat.) \pm 0.5 (syst.) & $7.05 \pm 0.01$ & $12.60 \pm 0.18$ \\
$B_{\tau}$ & $7.0 \pm 0.3$ (stat.) \pm 0.5 (syst.) & $7.20 \pm 0.13$ \\
\hline \hline
\end{tabular}

section of $178 \pm 17 \mathrm{pb}$ is in good agreement with those obtained by ATLAS for individual channels [52-54]. The selection criteria for this measurement were optimized for the $t \bar{t} \rightarrow \ell \tau_{\text {had }}+$ jets channel, which has the largest uncertainty, and then applied uniformly to all channels, ensuring no event overlap between them to exploit cancellation of systematic uncertainties in the ratios. This reduces the systematic uncertainties in the branching ratio measurements but it is not optimal for a cross-section measurement combining all channels. The systematic uncertainty on the inclusive cross section obtained by combining the samples used for this measurement is larger than the best ATLAS inclusive cross-section measurement [54], which achieved much smaller uncertainties because it was designed to minimize the systematic uncertainties related to jets, including the b-tagging efficiency and the jet energy scale. All cross-section measurements are in good agreement with the NNLO + NNLL theoretical prediction $177.3 \pm$ 9.0 $0_{-6.0}^{+4.6} \mathrm{pb}$ (calculated for a top mass of $172.5 \mathrm{GeV}[24,55]$ ).

The branching ratios into leptons and jets are in good agreement with the SM prediction that the top quark decays $100 \%$ to $W+$ quark. The precision of the measurements ranges from $2.3 \%$ for $B_{j}$ to $7.6 \%$ for $B_{\tau}$. The $B_{e}$ and $B_{\mu}$ include the leptonic decay of $\tau$ leptons while $B_{\tau}$ includes only the hadronic decays of $\tau$ leptons. There is no evidence for any non-SM top quark decay or for any non-SM process contribution that could affect these measurements. For example, the measured branching ratio $B_{\tau}$ will vary by more than the observed uncertainty if the branching ratio $\tilde{t} \rightarrow b \nu_{\tau} \tilde{\tau}$ times the $\tilde{t} \tilde{\bar{t}}$ production cross section $\left(\sigma_{\tilde{t} \tilde{\bar{t}}}\right)$ is greater than $3 \%$ of $\sigma_{t \bar{t}}$. The predicted $\sigma_{\tilde{t} \tilde{\bar{t}}}$ depends on $\tilde{t}$ mass $\left(m_{\tilde{t}}\right)$; it is equal to $\sigma_{t \bar{t}}$ for $m_{\tilde{t}}=120 \mathrm{GeV}$ and $12 \%$ of $\sigma_{t \bar{t}}$ for $m_{\tilde{t}}=180 \mathrm{GeV}[56]$.

\section{CONCLUSION}

The inclusive cross section for producing $t \bar{t}$ pairs in $p p$ collisions at a center-of-mass energy of $\sqrt{s}=7 \mathrm{TeV}$ at the LHC has been measured with the ATLAS detector and an integrated luminosity of $4.6 \mathrm{fb}^{-1}$ using the $\ell \tau_{\text {had }}+$ jets channel alone, as $\sigma_{t \bar{t}}=183 \pm 23 \mathrm{pb}$, and as a single parameter to fit the channels $\ell+$ jets, $\ell \ell+$ jets and $\ell \tau_{\text {had }}+$ jets, to be $178 \pm 17 \mathrm{pb}$. These are in agreement with all other cross-section measurements obtained by ATLAS and CMS. All cross-section measurements are fully compatible with the NNLO + NNLL theoretical prediction. Top quark branching ratios have also been measured and found to be in good agreement with branching ratios predicted by the SM. The precision ranges from $2.3 \%$ for the decays to jets to $7.6 \%$ for the decays to $\tau \nu+$ jet. There is no evidence for any non-SM process affecting these branching ratios.

\section{ACKNOWLEDGMENTS}

We thank CERN for the very successful operation of the LHC, as well as the support staff from our institutions without whom ATLAS could not be operated efficiently. We acknowledge the support of ANPCyT, Argentina; YerPhI, Armenia; ARC, Australia; BMWFW and FWF, Austria; ANAS, Azerbaijan; SSTC, Belarus; CNPq and FAPESP, Brazil; NSERC, NRC and CFI, Canada; CERN; CONICYT, Chile; CAS, MOST and NSFC, China; COLCIENCIAS, Colombia; MSMT CR, MPO CR and VSC CR, Czech Republic; DNRF, DNSRC and Lundbeck Foundation, Denmark; EPLANET, ERC and NSRF, European Union; IN2P3-CNRS, CEA-DSM/IRFU, France; GNSF, Georgia; BMBF, DFG, HGF Helmholtz Association, MPG and AvH Foundation, Germany; GSRT and NSRF, Greece; RGC, Hong Kong SAR, China; ISF, MINERVA, GIF, I-CORE and Benoziyo Center, Israel; INFN, Italy; MEXT and JSPS, Japan; CNRST, Morocco; FOM and NWO, Netherlands; BRF and RCN, Norway; MNiSW and NCN, Poland; GRICES and FCT, Portugal; MNE/IFA, Romania; MES of Russia and NRC KI, Russian Federation; JINR; MSTD, Serbia; MSSR, Slovakia; ARRS and MIZŠ, Slovenia; DST/NRF, South Africa; MINECO, Spain; SRC and Wallenberg Foundation, Sweden; SER, SNSF and Cantons of Bern and Geneva, Switzerland; NSC, 
Taiwan; TAEK, Turkey; STFC, the Royal Society and Leverhulme Trust, United Kingdom; DOE and NSF, United States of America. The crucial computing support from all WLCG partners is acknowledged gratefully, in particular from CERN and the ATLAS Tier-1 facilities at TRIUMF
(Canada), NDGF (Denmark, Norway, Sweden), CC-IN2P3 (France), KIT/GridKA (Germany), INFN-CNAF (Italy), NL-T1 (Netherlands), PIC (Spain), ASGC (Taiwan), RAL (UK) and BNL (USA) and in the Tier-2 facilities worldwide.
[1] CMS Collaboration, Phys. Lett. B 736, 33 (2014).

[2] ATLAS Collaboration, Phys. Rev. D 90, 112006 (2014).

[3] CMS Collaboration, J. High Energy Phys. 06 (2014) 090.

[4] T. Aaltonen et al. (CDF and D0 Collaborations), arXiv: 1503.05027.

[5] H. Baer, C. H. Chen, M. Drees, F. Paige, and X. Tata, Phys. Rev. Lett. 79, 986 (1997).

[6] T. Aaltonen et al. (CDF Collaboration), Phys. Rev. Lett. 103, 101803 (2009).

[7] V. M. Abazov et al. (DØ Collaboration), Phys. Lett. B 682, 278 (2009).

[8] V. M. Abazov et al. (DØ Collaboration), Phys. Rev. D 80, 071102 (2009).

[9] ATLAS Collaboration, Eur. Phys. J. C 73, 2465 (2013).

[10] ATLAS Collaboration, J. High Energy Phys. 03 (2015) 088.

[11] CMS Collaboration, J. High Energy Phys. 07 (2012) 143.

[12] G. F. Giudice and R. Rattazi, Phys. Rep. 322, 419 (1999).

[13] ALEPH, DELPHI, L3 and OPAL Collaborations and LEP Electroweak Working group, arXiv:hep-ex/0612034.

[14] K. G. Chetyrkin, J. H. Kuhn, and A. Kwiatowski, Phys. Rep. 277, 189 (1996).

[15] ATLAS Collaboration, Phys. Lett. B 717, 89 (2012).

[16] CMS Collaboration, Phys. Rev. D 85, 112007 (2012).

[17] B. P. Roe, H.-J. Yang, J. Zhu, Y. Liu, I. Stancu, and G. McGregor, Nucl. Instrum. Methods Phys. Res., Sect. A 543, 577 (2005).

[18] ATLAS Collaboration, J. Instrum. 3, S08003 (2008).

[19] S. Frixione, P. Nason, and C. Oleari, J. High Energy Phys. 11 (2007) 070.

[20] S. Alioli, P. Nason, C. Oleari, and E. Re, J. High Energy Phys. 06 (2010) 043.

[21] T. Sjostrand, S. Mrenna, and P. Skands, J. High Energy Phys. 05 (2006) 026.

[22] P. Z. Skands, Phys. Rev. D 82, 074018 (2010).

[23] P. M. Nadolsky, H.-L. Lai, Q.-H. Cao, J. Huston, J. Pumplin, D. Stump, Wu-Ki Tung and C.-P. Yuan, Phys. Rev. D 78, 013004 (2008).

[24] M. Czakon, P. Fiedler, and A. Mitov, Phys. Rev. Lett. 110, 252004 (2013).

[25] S. Frixione, E. Laenen, and B. R. Webber, J. High Energy Phys. 03 (2006) 092.

[26] G. Corcella, I. G Knowles, G. Marchesini, S. Moretti, K. Odagiri, P. Richardson, M. H. Seymour and B. R. Webber, J. High Energy Phys. 01 (2001) 010.

[27] J. M. Butterworth, J. R. Forshaw and M. H. Seymour, Z. Phys. C 72, 637 (1996).
[28] H. L. Lai, M. Guzzi. J. Huston, Z. Li, P. M. Nadolsky, J. Pumplin and C.-P. Yuan, Phys. Rev. D 82, 074024 (2010).

[29] M. L. Mangano, F. Piccinini, A. D. Polosa, M. Moretti and R. Pittau, J. High Energy Phys. 07 (2003) 001.

[30] J. Pumplin, D. R. Stump, J. Huston, H.-L. Lai, P. Nadolsky and W.-Ki Tung, J. High Energy Phys. 07 (2002) 012.

[31] J. Alwall et al., Eur. Phys. J. C 53, 473 (2008).

[32] A. Sherstnev and R. S. Thorne, Eur. Phys. J. C 55, 553 (2008).

[33] N. Davidson, G. Nanava, T. Przedziński, E. Richter-Wąs, and Z. Wąs, Comput. Phys. Commun. 183, 821 (2012).

[34] S. Agostinelli et al., Nucl. Instrum. Methods Phys. Res., Sect. A 506, 250 (2003).

[35] ATLAS Collaboration, Eur. Phys. J. C 70, 823 (2010).

[36] ATLAS Collaboration, Eur. Phys. J. C 74, 3130 (2014).

[37] ATLAS Collaboration, Eur. Phys. J. C 74, 2941 (2014).

[38] M. Cacciari, G. P. Salam, and G. Soyez, J. High Energy Phys. 04 (2008) 063.

[39] ATLAS Collaboration, Eur. Phys. J. C 75, 17 (2015).

[40] ATLAS Collaboration, Report No. ATLAS-CONF-2012043, 2012, https://cdsweb.cern.ch/record/1435197.

[41] C. Issever, K. Borras, and D. Wegener, Nucl. Instrum. Methods Phys. Res., Sect. A 545, 803 (2005).

[42] ATLAS Collaboration, Eur. Phys. J. C 73, 2304 (2013).

[43] ATLAS Collaboration, Eur. Phys. J. C 75, 303 (2015).

[44] R. Brun et al., https://root.cern.ch/root/html526/TH1.html/ \#TH1:KolmogorovTest.

[45] M. Botje et al., arXiv:1101.0538.

[46] A. Martin, W. Stirling, R. Thorne, and G. Watt, Eur. Phys. J. C 63, 189 (2009).

[47] R. D. Ball, L. D. Debbio, S. Forte, A. Guffanti, J. I. Latorre, A. Piccione, J. Rojo and M. Ubiali, Nucl. Phys. B809, 1 (2009).

[48] R. D. Ball, L. D. Debbio, S. Forte A. Guffanti, J. I. Latorre, A. Piccione, J. Rojo, and M. Ubiali, Nucl. Phys. B823, 195 (2009).

[49] ATLAS Collaboration, Eur. Phys. J. C 72, 2043 (2012).

[50] ATLAS Collaboration, Phys. Rev. D 85, 072004 (2012).

[51] ATLAS Collaboration, Eur. Phys. J. C 73, 2518 (2013).

[52] ATLAS Collaboration, Phys. Lett. B 711, 244 (2012).

[53] ATLAS Collaboration, J. High Energy Phys. 05 (2012) 059.

[54] ATLAS Collaboration, Eur. Phys. J. C 74, 3109 (2014).

[55] M. Czakon and A. Mitov, Comput. Phys. Commun. 185, 2930 (2014).

[56] W. Beenakker, S. Brensing, M. Krämernakker, A. Kuleszaker, E. Laenen and I. Niessen, J. High Energy Phys. 08 (2010) 098. 
G. Aad ${ }^{85}$ B. Abbott, ${ }^{113}$ J. Abdallah, ${ }^{151}$ O. Abdinov, ${ }^{11}$ R. Aben, ${ }^{107}$ M. Abolins, ${ }^{90}$ O. S. AbouZeid, ${ }^{158}$ H. Abramowicz, ${ }^{153}$ H. Abreu, ${ }^{152}$ R. Abreu, ${ }^{30}$ Y. Abulaiti, ${ }^{146 a, 146 b}$ B. S. Acharya, ${ }^{164 a, 164 b, b}$ L. Adamczyk, ${ }^{38 a}$ D. L. Adams, ${ }^{25}$ J. Adelman, ${ }^{108}$ S. Adomeit, ${ }^{100}$ T. Adye, ${ }^{131}$ A. A. Affolder, ${ }^{74}$ T. Agatonovic-Jovin, ${ }^{13}$ J. A. Aguilar-Saavedra, ${ }^{126 a, 126 f}$ S. P. Ahlen, ${ }^{22}$ F. Ahmadov ${ }^{65, c}$ G. Aielli, ${ }^{133 a, 133 b}$ H. Akerstedt, ${ }^{146 a, 146 b}$ T. P. A. Åkesson, ${ }^{81}$ G. Akimoto, ${ }^{155}$ A. V. Akimov, ${ }^{96}$ G. L. Alberghi, ${ }^{20 a, 20 b}$ J. Albert, ${ }^{169}$ S. Albrand, ${ }^{55}$ M. J. Alconada Verzini, ${ }^{71}$ M. Aleksa, ${ }^{30}$ I. N. Aleksandrov, ${ }^{65}$ C. Alexa, ${ }^{26 a}$ G. Alexander, ${ }^{153}$ T. Alexopoulos, ${ }^{10}$ M. Alhroob, ${ }^{113}$ G. Alimonti,${ }^{11 a}$ L. Alio, ${ }^{85}$ J. Alison, ${ }^{31}$ S. P. Alkire, ${ }^{35}$

B. M. M. Allbrooke, ${ }^{18}$ P. P. Allport, ${ }^{74}$ A. Aloisio, ${ }^{104 a, 104 b}$ A. Alonso, ${ }^{36}$ F. Alonso, ${ }^{71}$ C. Alpigiani, ${ }^{76}$ A. Altheimer, ${ }^{35}$ B. Alvarez Gonzalez,${ }^{30}$ D. Álvarez Piqueras, ${ }^{167}$ M. G. Alviggi, ${ }^{104 a, 104 b}$ B. T. Amadio, ${ }^{15}$ K. Amako, ${ }^{66}$ Y. Amaral Coutinho, ${ }^{24 a}$ C. Amelung, ${ }^{23}$ D. Amidei, ${ }^{89}$ S. P. Amor Dos Santos, ${ }^{126 a, 126 c}$ A. Amorim, ${ }^{126 a, 126 b}$ S. Amoroso, ${ }^{48}$ N. Amram, ${ }^{153}$ G. Amundsen, ${ }^{23}$ C. Anastopoulos, ${ }^{139}$ L. S. Ancu, ${ }^{49}$ N. Andari, ${ }^{30}$ T. Andeen, ${ }^{35}$ C. F. Anders, ${ }^{58 b}$ G. Anders,${ }^{30}$ J. K. Anders,${ }^{74}$ K. J. Anderson, ${ }^{31}$ A. Andreazza, ${ }^{91 \mathrm{a}, 91 \mathrm{~b}}$ V. Andrei ${ }^{58 \mathrm{a}}$ S. Angelidakis, ${ }^{9}$ I. Angelozzi,${ }^{107}$ P. Anger, ${ }^{44}$ A. Angerami ${ }^{35}$ F. Anghinolfi, ${ }^{30}$ A. V. Anisenkov, ${ }^{109, \mathrm{~d}}$ N. Anjos, ${ }^{12}$ A. Annovi, ${ }^{124 a, 124 b}$ M. Antonelli, ${ }^{47}$ A. Antonov, ${ }^{98}$ J. Antos, ${ }^{144 b}$ F. Anulli, ${ }^{132 a}$ M. Aoki, ${ }^{66}$ L. Aperio Bella, ${ }^{18}$ G. Arabidze,${ }^{90}$ Y. Arai ${ }^{66}$ J. P. Araque, ${ }^{126 a}$ A. T. H. Arce, ${ }^{45}$ F. A. Arduh, ${ }^{71}$ J-F. Arguin, ${ }^{95}$ S. Argyropoulos, ${ }^{42}$ M. Arik, ${ }^{19 a}$ A. J. Armbruster, ${ }^{30}$ O. Arnaez,${ }^{30}$ V. Arnal, ${ }^{82}$ H. Arnold,${ }^{48}$ M. Arratia, ${ }^{28}$ O. Arslan, ${ }^{21}$ A. Artamonov, ${ }^{97}$ G. Artoni, ${ }^{23}$ S. Asai, ${ }^{155}$ N. Asbah, ${ }^{42}$ A. Ashkenazi, ${ }^{153}$ B. Åsman, ${ }^{146 a, 146 b}$ L. Asquith, ${ }^{149}$ K. Assamagan, ${ }^{25}$ R. Astalos, ${ }^{144 a}$ M. Atkinson, ${ }^{165}$ N. B. Atlay, ${ }^{141}$ B. Auerbach, ${ }^{6}$ K. Augsten, ${ }^{128}$ M. Aurousseau, ${ }^{145 b}$ G. Avolio, ${ }^{30}$ B. Axen,${ }^{15}$ M. K. Ayoub, ${ }^{117}$ G. Azuelos, ${ }^{95, e}$ M. A. Baak, ${ }^{30}$ A. E. Baas, ${ }^{58 a}$ C. Bacci, ${ }^{134 a, 134 b}$ H. Bachacou, ${ }^{136}$ K. Bachas, ${ }^{154}$ M. Backes ${ }^{30}$ M. Backhaus ${ }^{30}$ P. Bagiacchi, ${ }^{132 a, 132 b}$ P. Bagnaia, ${ }^{132 a, 132 b}$ Y. Bai, ${ }^{33 a}$ T. Bain, ${ }^{35}$ J. T. Baines, ${ }^{131}$ O. K. Baker, ${ }^{176}$ P. Balek, ${ }^{129}$ T. Balestri, ${ }^{148}$ F. Balli, ${ }^{84}$ E. Banas, ${ }^{39}$ Sw. Banerjee,${ }^{173}$ A. A. E. Bannoura,${ }^{175}$ H. S. Bansil, ${ }^{18}$ L. Barak ${ }^{30}$ E. L. Barberio, ${ }^{88}$ D. Barberis, ${ }^{50,50 \mathrm{~b}}$ M. Barbero, ${ }^{85}$ T. Barillari, ${ }^{101}$ M. Barisonzi, ${ }^{164 a, 164 \mathrm{~b}}$ T. Barklow, ${ }^{143}$ N. Barlow, ${ }^{28}$ S. L. Barnes, ${ }^{84}$ B. M. Barnett, ${ }^{131}$ R. M. Barnett, ${ }^{15}$ Z. Barnovska, ${ }^{5}$ A. Baroncelli, ${ }^{134 a}$ G. Barone,${ }^{49}$ A. J. Barr, ${ }^{120}$ F. Barreiro, ${ }^{82}$ J. Barreiro Guimarães da Costa, ${ }^{57}$ R. Bartoldus, ${ }^{143}$ A. E. Barton,${ }^{72}$ P. Bartos,${ }^{144 a}$ A. Basalaev, ${ }^{123}$ A. Bassalat, ${ }^{117}$ A. Basye, ${ }^{165}$ R. L. Bates, ${ }^{53}$ S. J. Batista, ${ }^{158}$ J. R. Batley, ${ }^{28}$ M. Battaglia, ${ }^{137}$ M. Bauce,${ }^{132 a, 132 b}$ F. Bauer, ${ }^{136}$ H. S. Bawa, ${ }^{143, f}$ J. B. Beacham, ${ }^{111}$ M. D. Beattie, ${ }^{72}$ T. Beau, ${ }^{80}$ P. H. Beauchemin, ${ }^{161}$ R. Beccherle, ${ }^{124 a, 124 b}$ P. Bechtle, ${ }^{21}$ H. P. Beck, ${ }^{17, g}$ K. Becker, ${ }^{120}$ M. Becker ${ }^{83}$ S. Becker, ${ }^{100}$ M. Beckingham, ${ }^{170}$ C. Becot,${ }^{117}$ A. J. Beddall, ${ }^{19 \mathrm{c}}$ A. Beddall, ${ }^{19 \mathrm{c}}$ V. A. Bednyakov, ${ }^{65}$ C. P. Bee, ${ }^{148}$ L. J. Beemster, ${ }^{107}$ T. A. Beermann, ${ }^{175}$ M. Begel,${ }^{25}$ J. K. Behr, ${ }^{120}$ C. Belanger-Champagne, ${ }^{87}$ W. H. Bell, ${ }^{49}$

G. Bella, ${ }^{153}$ L. Bellagamba, ${ }^{20 a}$ A. Bellerive, ${ }^{29}$ M. Bellomo, ${ }^{86}$ K. Belotskiy, ${ }^{98}$ O. Beltramello, ${ }^{30}$ O. Benary, ${ }^{153}$

D. Benchekroun, ${ }^{135 a}$ M. Bender, ${ }^{100}$ K. Bendtz, ${ }^{146 a, 146 b}$ N. Benekos, ${ }^{10}$ Y. Benhammou, ${ }^{153}$ E. Benhar Noccioli, ${ }^{49}$ J. A. Benitez Garcia, ${ }^{159 b}$ D. P. Benjamin, ${ }^{45}$ J. R. Bensinger, ${ }^{23}$ S. Bentvelsen, ${ }^{107}$ L. Beresford, ${ }^{120}$ M. Beretta, ${ }^{47}$ D. Berge, ${ }^{107}$ E. Bergeaas Kuutmann, ${ }^{166}$ N. Berger, ${ }^{5}$ F. Berghaus, ${ }^{169}$ J. Beringer, ${ }^{15}$ C. Bernard, ${ }^{22}$ N. R. Bernard, ${ }^{86}$ C. Bernius, ${ }^{110}$ F. U. Bernlochner, ${ }^{21}$ T. Berry, ${ }^{77}$ P. Berta, ${ }^{129}$ C. Bertella, ${ }^{83}$ G. Bertoli, ${ }^{146 a, 146 b}$ F. Bertolucci, ${ }^{124 a, 124 b}$ C. Bertsche, ${ }^{113}$ D. Bertsche, ${ }^{113}$ M. I. Besana, ${ }^{91 a}$ G. J. Besjes, ${ }^{106}$ O. Bessidskaia Bylund,${ }^{146 a, 146 b}$ M. Bessner, ${ }^{42}$ N. Besson, ${ }^{136}$ C. Betancourt ${ }^{48}$ S. Bethke, ${ }^{101}$ A. J. Bevan, ${ }^{76}$ W. Bhimji, ${ }^{46}$ R. M. Bianchi, ${ }^{125}$ L. Bianchini, ${ }^{23}$ M. Bianco, ${ }^{30}$ O. Biebel, ${ }^{100}$ D. Biedermann, ${ }^{16}$ S. P. Bieniek, ${ }^{78}$ M. Biglietti, ${ }^{134 a}$ J. Bilbao De Mendizabal, ${ }^{49}$ H. Bilokon,${ }^{47}$ M. Bindi, ${ }^{54}$ S. Binet,${ }^{117}$ A. Bingul, ${ }^{19 c}$ C. Bini, ${ }^{132 a, 132 b}$ C. W. Black, ${ }^{150}$ J. E. Black, ${ }^{143}$ K. M. Black, ${ }^{22}$ D. Blackburn, ${ }^{138}$ R. E. Blair, ${ }^{6}$ J.-B. Blanchard, ${ }^{136}$ J. E. Blanco, ${ }^{77}$ T. Blazek, ${ }^{144 a}$ I. Bloch ${ }^{42}$ C. Blocker ${ }^{23}$ W. Blum, ${ }^{83, a}$ U. Blumenschein, ${ }^{54}$ G. J. Bobbink, ${ }^{107}$

V. S. Bobrovnikov, ${ }^{109, \mathrm{~d}}$ S. S. Bocchetta, ${ }^{81}$ A. Bocci, ${ }^{45}$ C. Bock, ${ }^{100}$ M. Boehler, ${ }^{48}$ J. A. Bogaerts, ${ }^{30}$ D. Bogavac, ${ }^{13}$ A. G. Bogdanchikov, ${ }^{109}$ C. Bohm, ${ }^{146 a}$ V. Boisvert, ${ }^{77}$ T. Bold, ${ }^{38 a}$ V. Boldea, ${ }^{26 a}$ A. S. Boldyrev, ${ }^{99}$ M. Bomben,${ }^{80}$ M. Bona,${ }^{76}$ M. Boonekamp, ${ }^{136}$ A. Borisov, ${ }^{130}$ G. Borissov, ${ }^{72}$ S. Borroni, ${ }^{42}$ J. Bortfeldt, ${ }^{100}$ V. Bortolotto,${ }^{60 a, 60 b, 60 \mathrm{c}} \mathrm{K}$. Bos, ${ }^{107}$ D. Boscherini, ${ }^{20 a}$ M. Bosman, ${ }^{12}$ J. Boudreau, ${ }^{125}$ J. Bouffard, ${ }^{2}$ E. V. Bouhova-Thacker, ${ }^{72}$ D. Boumediene,${ }^{34}$ C. Bourdarios, ${ }^{117}$ N. Bousson, ${ }^{114}$ A. Boveia, ${ }^{30}$ J. Boyd ${ }^{30}$ I. R. Boyko, ${ }^{65}$ I. Bozic, ${ }^{13}$ J. Bracinik,${ }^{18}$ A. Brandt, ${ }^{8}$ G. Brandt, ${ }^{54}$ O. Brandt ${ }^{58 a}$ U. Bratzler, ${ }^{156}$ B. Brau, ${ }^{86}$ J. E. Brau, ${ }^{116}$ H. M. Braun, ${ }^{175, a}$ S. F. Brazzale, ${ }^{164 a, 164 c}$ W. D. Breaden Madden, ${ }^{53}$ K. Brendlinger, ${ }^{122}$ A. J. Brennan, ${ }^{88}$ L. Brenner, ${ }^{107}$ R. Brenner, ${ }^{166}$ S. Bressler, ${ }^{172}$ K. Bristow, ${ }^{145}$ T. M. Bristow, ${ }^{46}$ D. Britton, ${ }^{53}$ D. Britzger, ${ }^{42}$ F. M. Brochu, ${ }^{28}$ I. Brock, ${ }^{21}$ R. Brock,${ }^{90}$ J. Bronner, ${ }^{101}$ G. Brooijmans, ${ }^{35}$ T. Brooks, ${ }^{77}$ W. K. Brooks,${ }^{32 b}$ J. Brosamer, ${ }^{15}$ E. Brost ${ }^{116}$ J. Brown, ${ }^{55}$ P. A. Bruckman de Renstrom, ${ }^{39}$ D. Bruncko, ${ }^{144 \mathrm{~b}}$ R. Bruneliere, ${ }^{48}$ A. Bruni, ${ }^{20 \mathrm{a}}$ G. Bruni, ${ }^{20 \mathrm{a}}$ M. Bruschi, ${ }^{20 \mathrm{a}}$ N. Bruscino, ${ }^{21}$ L. Bryngemark, ${ }^{81}$ T. Buanes,${ }^{14}$ Q. Buat, ${ }^{142}$ P. Buchholz, ${ }^{141}$ A. G. Buckley, ${ }^{53}$ S. I. Buda, ${ }^{26 a}$ I. A. Budagov, ${ }^{65}$ F. Buehrer, ${ }^{48}$ L. Bugge, ${ }^{119}$ M. K. Bugge, ${ }^{119}$ O. Bulekov, ${ }^{98}$ D. Bullock, ${ }^{8}$ H. Burckhart, ${ }^{30}$ S. Burdin, ${ }^{74}$ B. Burghgrave, ${ }^{108}$ S. Burke, ${ }^{131}$ I. Burmeister, ${ }^{43}$ E. Busato, ${ }^{34}$ D. Büscher, ${ }^{48}$ V. Büscher, ${ }^{83}$ P. Bussey, ${ }^{53}$ J. M. Butler, ${ }^{22}$ 
A. I. Butt, ${ }^{3}$ C. M. Buttar, ${ }^{53}$ J. M. Butterworth ${ }^{78}$ P. Butti, ${ }^{107}$ W. Buttinger, ${ }^{25}$ A. Buzatu, ${ }^{53}$ A. R. Buzykaev, ${ }^{109, d}$ S. Cabrera Urbán, ${ }^{167}$ D. Caforio, ${ }^{128}$ V. M. Cairo, ${ }^{37 a, 37 b}$ O. Cakir, ${ }^{4 a}$ P. Calafiura, ${ }^{15}$ A. Calandri, ${ }^{136}$ G. Calderini ${ }^{80}$ P. Calfayan, ${ }^{100}$ L. P. Caloba, ${ }^{24 a}$ D. Calvet,${ }^{34}$ S. Calvet,${ }^{34}$ R. Camacho Toro, ${ }^{31}$ S. Camarda, ${ }^{42}$ P. Camarri, ${ }^{133 a, 133 b}$ D. Cameron, ${ }^{119}$ R. Caminal Armadans, ${ }^{165}$ S. Campana,${ }^{30}$ M. Campanelli, ${ }^{78}$ A. Campoverde, ${ }^{148}$ V. Canale, ${ }^{104 a, 104 b}$ A. Canepa, ${ }^{159 a}$ M. Cano Bret, ${ }^{76}$ J. Cantero, ${ }^{82}$ R. Cantrill, ${ }^{126 a}$ T. Cao, ${ }^{40}$ M. D. M. Capeans Garrido, ${ }^{30}$ I. Caprini, ${ }^{26 a}$ M. Caprini, ${ }^{26 a}$ M. Capua, ${ }^{37 a, 37 b}$ R. Caputo,${ }^{83}$ R. Cardarelli, ${ }^{133 a}$ F. Cardillo, ${ }^{48}$ T. Carli, ${ }^{30}$ G. Carlino, ${ }^{104 a}$ L. Carminati, ${ }^{91,91 b}$ S. Caron, ${ }^{106}$ E. Carquin, ${ }^{32 \mathrm{a}}$ G. D. Carrillo-Montoya, ${ }^{8}$ J. R. Carter, ${ }^{28}$ J. Carvalho, ${ }^{126 a, 126 c}$ D. Casadei, ${ }^{78}$ M. P. Casado, ${ }^{12}$ M. Casolino, ${ }^{12}$ E. Castaneda-Miranda, ${ }^{145 b}$ A. Castelli, ${ }^{107}$ V. Castillo Gimenez,${ }^{167}$ N. F. Castro, ${ }^{126 a, h}$ P. Catastini, ${ }^{57}$ A. Catinaccio, ${ }^{30}$ J. R. Catmore, ${ }^{119}$ A. Cattai, ${ }^{30}$ J. Caudron, ${ }^{83}$ V. Cavaliere, ${ }^{165}$ D. Cavalli, ${ }^{91 a}$ M. Cavalli-Sforza, ${ }^{12}$ V. Cavasinni, ${ }^{124 a, 124 b}$ F. Ceradini, ${ }^{134 a, 134 b}$ B. C. Cerio, ${ }^{45}$ K. Cerny, ${ }^{129}$ A. S. Cerqueira, ${ }^{24 b}$ A. Cerri, ${ }^{149}$ L. Cerrito, ${ }^{76}$ F. Cerutti, ${ }^{15}$ M. Cerv, ${ }^{30}$ A. Cervelli, ${ }^{17}$ S. A. Cetin, ${ }^{19 b}$ A. Chafaq, ${ }^{135 a}$ D. Chakraborty, ${ }^{108}$ I. Chalupkova, ${ }^{129}$ P. Chang, ${ }^{165}$ B. Chapleau, ${ }^{87}$ J. D. Chapman, ${ }^{28}$ D. G. Charlton, ${ }^{18}$ C. C. Chau, ${ }^{158}$ C. A. Chavez Barajas, ${ }^{149}$ S. Cheatham, ${ }^{152}$ A. Chegwidden, ${ }^{90}$ S. Chekanov, ${ }^{6}$ S. V. Chekulaev, ${ }^{159 a}$ G. A. Chelkov, ${ }^{65, i}$ M. A. Chelstowska,${ }^{89}$ C. Chen, ${ }^{64}$ H. Chen, ${ }^{25}$ K. Chen, ${ }^{148}$ L. Chen, ${ }^{33 d, j}$ S. Chen, ${ }^{33 \mathrm{c}}$ X. Chen, ${ }^{33 \mathrm{f}}$ Y. Chen, ${ }^{67}$ H. C. Cheng, ${ }^{89}$ Y. Cheng, ${ }^{31}$ A. Cheplakov, ${ }^{65}$ E. Cheremushkina, ${ }^{130}$ R. Cherkaoui El Moursli, ${ }^{135 e}$ V. Chernyatin, ${ }^{25, a}$ E. Cheu, ${ }^{7}$ L. Chevalier, ${ }^{136}$ V. Chiarella, ${ }^{47}$ J. T. Childers, ${ }^{6}$ G. Chiodini, ${ }^{73 a}$ A. S. Chisholm, ${ }^{18}$ R. T. Chislett, ${ }^{78}$ A. Chitan, ${ }^{26 a}$ M. V. Chizhov, ${ }^{65}$ K. Choi, ${ }^{61}$ S. Chouridou, ${ }^{9}$ B. K. B. Chow, ${ }^{100}$ V. Christodoulou, ${ }^{78}$ D. Chromek-Burckhart, ${ }^{30}$ J. Chudoba, ${ }^{127}$ A. J. Chuinard, ${ }^{87}$ J. J. Chwastowski ${ }^{39}$ L. Chytka, ${ }^{115}$ G. Ciapetti, ${ }^{132 a, 132 b}$ A. K. Ciftci, ${ }^{4 a}$ D. Cinca, ${ }^{53}$ V. Cindro, ${ }^{75}$ I. A. Cioara, ${ }^{21}$ A. Ciocio, ${ }^{15}$ Z. H. Citron, ${ }^{172}$ M. Ciubancan, ${ }^{26 a}$ A. Clark, ${ }^{49}$ B. L. Clark, ${ }^{57}$ P. J. Clark, ${ }^{46}$ R. N. Clarke, ${ }^{15}$ W. Cleland, ${ }^{125}$ C. Clement, ${ }^{146 a, 146 b}$ Y. Coadou, ${ }^{85}$ M. Cobal, ${ }^{164 a, 164 \mathrm{c}}$ A. Coccaro, ${ }^{138}$ J. Cochran, ${ }^{64}$ L. Coffey, ${ }^{23}$ J. G. Cogan, ${ }^{143}$ B. Cole, ${ }^{35}$ S. Cole,,${ }^{108}$ A. P. Colijn, ${ }^{107}$ J. Collot, ${ }^{55}$ T. Colombo, ${ }^{58 c}$ G. Compostella, ${ }^{101}$ P. Conde Muiño, ${ }^{126 a, 126 b}$ E. Coniavitis, ${ }^{48}$ S. H. Connell, ${ }^{145 b}$ I. A. Connelly, ${ }^{77}$ S. M. Consonni, ${ }^{91 a, 91 b}$ V. Consorti, ${ }^{48}$ S. Constantinescu, ${ }^{26 a}$ C. Conta, ${ }^{121 a, 121 b}$ G. Conti, ${ }^{30}$ F. Conventi, ${ }^{104 a, k}$ M. Cooke, ${ }^{15}$ B. D. Cooper, ${ }^{78}$ A. M. Cooper-Sarkar, ${ }^{120}$ T. Cornelissen, ${ }^{175}$ M. Corradi, ${ }^{20 a}$ F. Corriveau, ${ }^{87,1}$ A. Corso-Radu ${ }^{163}$ A. Cortes-Gonzalez, ${ }^{12}$ G. Cortiana, ${ }^{101}$ G. Costa, ${ }^{91 a}$ M. J. Costa, ${ }^{167}$ D. Costanzo, ${ }^{139}$ D. Côté,${ }^{8}$ G. Cottin,${ }^{28}$ G. Cowan, ${ }^{77}$ B. E. Cox,${ }^{84}$ K. Cranmer, ${ }^{110}$ G. Cree, ${ }^{29}$ S. Crépé-Renaudin, ${ }^{55}$ F. Crescioli ${ }^{80}$ W. A. Cribbs, ${ }^{146 a, 146 b}$ M. Crispin Ortuzar, ${ }^{120}$ M. Cristinziani, ${ }^{21}$ V. Croft, ${ }^{106}$ G. Crosetti, ${ }^{37 \mathrm{a}, 37 \mathrm{~b}}$ T. Cuhadar Donszelmann, ${ }^{139} \mathrm{~J}$. Cummings, ${ }^{176}$ M. Curatolo, ${ }^{47}$ C. Cuthbert, ${ }^{150}$ H. Czirr, ${ }^{141}$ P. Czodrowski, ${ }^{3}$ S. D’Auria, ${ }^{53}$ M. D’Onofrio, ${ }^{74}$ M. J. Da Cunha Sargedas De Sousa, ${ }^{126 a, 126 b}$ C. Da Via, ${ }^{84}$ W. Dabrowski, ${ }^{38 a}$ A. Dafinca, ${ }^{120}$

T. Dai, ${ }^{89}$ O. Dale, ${ }^{14}$ F. Dallaire, ${ }^{95}$ C. Dallapiccola,${ }^{86}$ M. Dam, ${ }^{36}$ J. R. Dandoy,${ }^{31}$ N. P. Dang, ${ }^{48}$ A. C. Daniells, ${ }^{18}$ M. Danninger, ${ }^{168}$ M. Dano Hoffmann, ${ }^{136}$ V. Dao, ${ }^{48}$ G. Darbo, ${ }^{50 a}$ S. Darmora, ${ }^{8}$ J. Dassoulas, ${ }^{3}$ A. Dattagupta,${ }^{61}$ W. Davey, ${ }^{21}$ C. David, ${ }^{169}$ T. Davidek, ${ }^{129}$ E. Davies, ${ }^{120, \mathrm{~m}}$ M. Davies, ${ }^{153}$ P. Davison, ${ }^{78}$ Y. Davygora, ${ }^{58 \mathrm{a}}$ E. Dawe, ${ }^{88}$ I. Dawson, ${ }^{139}$ R. K. Daya-Ishmukhametova ${ }^{86}$ K. De,${ }^{8}$ R. de Asmundis, ${ }^{104 a}$ S. De Castro, ${ }^{20 a, 20 b}$ S. De Cecco, ${ }^{80}$ N. De Groot, ${ }^{106}$ P. de Jong, ${ }^{107}$ H. De la Torre, ${ }^{82}$ F. De Lorenzi, ${ }^{64}$ L. De Nooij, ${ }^{107}$ D. De Pedis, ${ }^{132 a}$ A. De Salvo, ${ }^{132 a}$ U. De Sanctis, ${ }^{149}$ A. De Santo, ${ }^{149}$ J. B. De Vivie De Regie, ${ }^{117}$ W. J. Dearnaley, ${ }^{72}$ R. Debbe,${ }^{25}$ C. Debenedetti, ${ }^{137}$ D. V. Dedovich,${ }^{65}$ I. Deigaard, ${ }^{107}$ J. Del Peso, ${ }^{82}$ T. Del Prete, ${ }^{124 a, 124 b}$ D. Delgove, ${ }^{117}$ F. Deliot, ${ }^{136}$ C. M. Delitzsch, ${ }^{49}$ M. Deliyergiyev,${ }^{75}$ A. Dell'Acqua, ${ }^{30}$ L. Dell'Asta, ${ }^{22}$ M. Dell'Orso,${ }^{124 a, 124 b}$ M. Della Pietra,${ }^{104 a, k}$ D. della Volpe,${ }^{49}$ M. Delmastro,${ }^{5}$ P. A. Delsart, ${ }^{55}$ C. Deluca, ${ }^{107}$ D. A. DeMarco, ${ }^{158}$ S. Demers, ${ }^{176}$ M. Demichev, ${ }^{65}$ A. Demilly, ${ }^{80}$ S. P. Denisov, ${ }^{130}$ D. Derendarz,${ }^{39}$ J. E. Derkaoui, ${ }^{135 d}$ F. Derue,${ }^{80}$ P. Dervan, ${ }^{74}$ K. Desch,,${ }^{21}$ C. Deterre, ${ }^{42}$ P. O. Deviveiros, ${ }^{30}$ A. Dewhurst,${ }^{131}$ S. Dhaliwal, ${ }^{23}$ A. Di Ciaccio, ${ }^{133 a, 133 b}$ L. Di Ciaccio, ${ }^{5}$ A. Di Domenico, ${ }^{132 a, 132 b}$ C. Di Donato, ${ }^{104 a, 104 b}$ A. Di Girolamo, ${ }^{30}$ B. Di Girolamo, ${ }^{30}$ A. Di Mattia ${ }^{152}$ B. Di Micco, ${ }^{134 a, 134 b}$ R. Di Nardo,${ }^{47}$ A. Di Simone,${ }^{48}$ R. Di Sipio, ${ }^{158}$ D. Di Valentino, ${ }^{29}$ C. Diaconu, ${ }^{85}$ M. Diamond, ${ }^{158}$ F. A. Dias, ${ }^{46}$ M. A. Diaz, ${ }^{32 a}$ E. B. Diehl, ${ }^{89}$ J. Dietrich, ${ }^{16}$ S. Diglio, ${ }^{85}$ A. Dimitrievska, ${ }^{13}$ J. Dingfelder, ${ }^{21}$ P. Dita, ${ }^{26 a}$ S. Dita, ${ }^{26 a}$ F. Dittus, ${ }^{30}$ F. Djama, ${ }^{85}$ T. Djobava, ${ }^{51 b}$ J. I. Djuvsland, ${ }^{58 a}$ M. A. B. do Vale, ${ }^{24 c}$ D. Dobos,${ }^{30}$ M. Dobre, ${ }^{26 a}$ C. Doglioni,${ }^{49}$ T. Dohmae, ${ }^{155}$ J. Dolejsi, ${ }^{129}$ Z. Dolezal, ${ }^{129}$ B. A. Dolgoshein, ${ }^{98, a}$ M. Donadelli, ${ }^{24 \mathrm{~d}}$ S. Donati, ${ }^{124 a, 124 b}$ P. Dondero, ${ }^{121 a, 121 b}$ J. Donini, ${ }^{34}$ J. Dopke, ${ }^{131}$ A. Doria, ${ }^{104 a}$ M. T. Dova, ${ }^{71}$ A. T. Doyle, ${ }^{53}$ E. Drechsler,${ }^{54}$ M. Dris, ${ }^{10}$ E. Dubreuil, ${ }^{34}$ E. Duchovni, ${ }^{172}$ G. Duckeck, ${ }^{100}$ O. A. Ducu, ${ }^{26 a, 85}$ D. Duda, ${ }^{175}$ A. Dudarev, ${ }^{30}$ L. Duflot, ${ }^{117}$ L. Duguid, ${ }^{77}$ M. Dührssen, ${ }^{30}$ M. Dunford ${ }^{58 a}$ H. Duran Yildiz,${ }^{4 a}$ M. Düren,${ }^{52}$ A. Durglishvili, ${ }^{51 b}$ D. Duschinger, ${ }^{44}$ M. Dyndal, ${ }^{38 a}$ C. Eckardt, ${ }^{42}$ K. M. Ecker, ${ }^{101}$ R. C. Edgar, ${ }^{89}$ W. Edson, ${ }^{2}$ N. C. Edwards, ${ }^{46}$ W. Ehrenfeld, ${ }^{21}$ T. Eifert, ${ }^{30}$ G. Eigen, ${ }^{14}$ K. Einsweiler, ${ }^{15}$ T. Ekelof ${ }^{166}$ M. El Kacimi, ${ }^{135 c}$ M. Ellert, ${ }^{166}$ S. Elles, ${ }^{5}$ F. Ellinghaus, ${ }^{83}$ A. A. Elliot, ${ }^{169}$ N. Ellis, ${ }^{30}$ J. Elmsheuser, ${ }^{100}$ M. Elsing, ${ }^{30}$ D. Emeliyanov, ${ }^{131}$ Y. Enari, ${ }^{155}$ O. C. Endner, ${ }^{83}$ M. Endo ${ }^{118}$ J. Erdmann ${ }^{43}$ A. Ereditato, ${ }^{17}$ G. Ernis, ${ }^{175}$ J. Ernst, ${ }^{2}$ M. Ernst, ${ }^{25}$ S. Errede, ${ }^{165}$ E. Ertel, ${ }^{83}$ M. Escalier, ${ }^{117}$ H. Esch, ${ }^{43}$ C. Escobar, ${ }^{125}$ B. Esposito, ${ }^{47}$ 
A. I. Etienvre, ${ }^{136}$ E. Etzion, ${ }^{153}$ H. Evans, ${ }^{61}$ A. Ezhilov, ${ }^{123}$ L. Fabbri, ${ }^{20 a, 20 b}$ G. Facini, ${ }^{31}$ R. M. Fakhrutdinov, ${ }^{130}$ S. Falciano, ${ }^{132 a}$ R. J. Falla, ${ }^{78}$ J. Faltova, ${ }^{129}$ Y. Fang, ${ }^{33 a}$ M. Fanti, ${ }^{91 a, 91 b}$ A. Farbin, ${ }^{8}$ A. Farilla, ${ }^{134 a}$ T. Farooque, ${ }^{12}$ S. Farrell, ${ }^{15}$

S. M. Farrington, ${ }^{170}$ P. Farthouat, ${ }^{30}$ F. Fassi, ${ }^{135 \mathrm{e}}$ P. Fassnacht, ${ }^{30}$ D. Fassouliotis, ${ }^{9}$ M. Faucci Giannelli, ${ }^{77}$ A. Favareto, ${ }^{50 a, 50 \mathrm{~b}}$ L. Fayard ${ }^{117}$ P. Federic, ${ }^{144 a}$ O. L. Fedin, ${ }^{123, n}$ W. Fedorko, ${ }^{168}$ S. Feigl,${ }^{30}$ L. Feligioni, ${ }^{85}$ C. Feng, ${ }^{33 d}$ E. J. Feng, ${ }^{6}$ H. Feng ${ }^{89}$ A. B. Fenyuk ${ }^{130}$ L. Feremenga, ${ }^{8}$ P. Fernandez Martinez, ${ }^{167}$ S. Fernandez Perez,${ }^{30}$ J. Ferrando, ${ }^{53}$ A. Ferrari, ${ }^{166}$ P. Ferrari, ${ }^{107}$ R. Ferrari, ${ }^{121 a}$ D. E. Ferreira de Lima, ${ }^{53}$ A. Ferrer,${ }^{167}$ D. Ferrere, ${ }^{49}$ C. Ferretti, ${ }^{89}$ A. Ferretto Parodi, ${ }^{50 a, 50 b}$ M. Fiascaris ${ }^{31}$ F. Fiedler ${ }^{83}$ A. Filipčič ${ }^{75}$ M. Filipuzzii, ${ }^{42}$ F. Filthaut, ${ }^{106}$ M. Fincke-Keeler,${ }^{169}$ K. D. Finelli, ${ }^{150}$ M. C. N. Fiolhais, ${ }^{126 a, 126 c}$ L. Fiorini, ${ }^{167}$ A. Firan, ${ }^{40}$ A. Fischer, ${ }^{2}$ C. Fischer, ${ }^{12}$ J. Fischer, ${ }^{175}$ W. C. Fisher,${ }^{90}$ E. A. Fitzgerald, ${ }^{23}$ N. Flaschel, ${ }^{42}$ I. Fleck, ${ }^{141}$ P. Fleischmann, ${ }^{89}$ S. Fleischmann, ${ }^{175}$ G. T. Fletcher ${ }^{139}$ G. Fletcher, ${ }^{76}$ R. R. M. Fletcher, ${ }^{122}$ T. Flick, ${ }^{175}$ A. Floderus,${ }^{81}$ L. R. Flores Castillo, ${ }^{60 a}$ M. J. Flowerdew, ${ }^{101}$ A. Formica, ${ }^{136}$ A. Forti, ${ }^{84}$ D. Fournier, ${ }^{117}$ H. Fox, ${ }^{72}$ S. Fracchia, ${ }^{12}$ P. Francavilla, ${ }^{80}$ M. Franchini, ${ }^{20 a, 20 b}$ D. Francis,${ }^{30}$ L. Franconi, ${ }^{119}$ M. Franklin, ${ }^{57}$ M. Frate, ${ }^{163}$ M. Fraternali, ${ }^{121 a, 121 b}$ D. Freeborn, ${ }^{78}$ S. T. French ${ }^{28}$ F. Friedrich, ${ }^{44}$ D. Froidevaux,${ }^{30}$ J. A. Frost,${ }^{120}$ C. Fukunaga, ${ }^{156}$ E. Fullana Torregrosa,${ }^{83}$ B. G. Fulsom, ${ }^{143}$ J. Fuster, ${ }^{167}$ C. Gabaldon, ${ }^{55}$ O. Gabizon, ${ }^{175}$ A. Gabrielli, ${ }^{20 a, 20 b}$ A. Gabrielli, ${ }^{132 a, 132 b}$ S. Gadatsch, ${ }^{107}$ S. Gadomski, ${ }^{49}$ G. Gagliardi, ${ }^{50,50 b}$ P. Gagnon, ${ }^{61}$ C. Galea, ${ }^{106}$ B. Galhardo, ${ }^{126 a, 126 c}$ E. J. Gallas, ${ }^{120}$ B. J. Gallop, ${ }^{131}$ P. Gallus, ${ }^{128}$ G. Galster, ${ }^{36}$ K. K. Gan, ${ }^{111}$ J. Gao, ${ }^{33 b, 85}$ Y. Gao, ${ }^{46}$ Y. S. Gao, ${ }^{143, f}$ F. M. Garay Walls, ${ }^{46}$ F. Garberson, ${ }^{176}$ C. García, ${ }^{167}$ J. E. García Navarro, ${ }^{167}$ M. Garcia-Sciveres, ${ }^{15}$ R. W. Gardner,${ }^{31}$ N. Garelli, ${ }^{143}$ V. Garonne,${ }^{119}$ C. Gatti, ${ }^{47}$ A. Gaudiello, ${ }^{50 a, 50 b}$ G. Gaudio, ${ }^{121 a}$ B. Gaur, ${ }^{141}$ L. Gauthier ${ }^{95}$ P. Gauzzi, ${ }^{132 a, 132 b}$ I. L. Gavrilenko, ${ }^{96}$ C. Gay ${ }^{168}$ G. Gaycken,${ }^{21}$ E. N. Gazis, ${ }^{10}$ P. Ge,${ }^{33 \mathrm{~d}}$ Z. Gecse, ${ }^{168}$ C. N. P. Gee,${ }^{131}$ D. A. A. Geerts, ${ }^{107}$ Ch. Geich-Gimbel,${ }^{21}$ M. P. Geisler, ${ }^{58 a}$ C. Gemme, ${ }^{50 a}$ M. H. Genest,${ }^{55}$ S. Gentile, ${ }^{132 a, 132 b}$ M. George,${ }^{54}$ S. George,${ }^{77}$ D. Gerbaudo, ${ }^{163}$ A. Gershon, ${ }^{153}$ H. Ghazlane, ${ }^{135 b}$ B. Giacobbe, ${ }^{20 \mathrm{a}}$ S. Giagu, ${ }^{132 a, 132 b}$ V. Giangiobbe, ${ }^{12}$ P. Giannetti, ${ }^{124 a, 124 b}$ B. Gibbard ${ }^{25}$ S. M. Gibson, ${ }^{77}$ M. Gilchriese, ${ }^{15}$ T. P. S. Gillam, ${ }^{28}$ D. Gillberg, ${ }^{30}$ G. Gilles, ${ }^{34}$ D. M. Gingrich,,${ }^{3, \mathrm{e}}$ N. Giokaris, ${ }^{9}$ M. P. Giordani, ${ }^{164 a, 164 \mathrm{c}}$ F. M. Giorgi, ${ }^{20 \mathrm{a}}$ F. M. Giorgi, ${ }^{16}$ P. F. Giraud, ${ }^{136}$ P. Giromini, ${ }^{47}$ D. Giugni, ${ }^{91 a}$ C. Giuliani, ${ }^{48}$ M. Giulini,${ }^{58 b}$ B. K. Gjelsten, ${ }^{119}$ S. Gkaitatzis, ${ }^{154}$

I. Gkialas, ${ }^{154}$ E. L. Gkougkousis, ${ }^{117}$ L. K. Gladilin, ${ }^{99}$ C. Glasman, ${ }^{82}$ J. Glatzer, ${ }^{30}$ P. C. F. Glaysher, ${ }^{46}$ A. Glazov, ${ }^{42}$ M. Goblirsch-Kolb, ${ }^{101}$ J. R. Goddard, ${ }^{76}$ J. Godlewski, ${ }^{39}$ S. Goldfarb ${ }^{89}$ T. Golling, ${ }^{49}$ D. Golubkov, ${ }^{130}$ A. Gomes, ${ }^{126 a, 126 b, 126 \mathrm{~d}}$ R. Gonçalo, ${ }^{126 a}$ J. Goncalves Pinto Firmino Da Costa, ${ }^{136}$ L. Gonella, ${ }^{21}$ S. González de la Hoz, ${ }^{167}$ G. Gonzalez Parra, ${ }^{12}$ S. Gonzalez-Sevilla, ${ }^{49}$ L. Goossens, ${ }^{30}$ P. A. Gorbounov, ${ }^{97}$ H. A. Gordon, ${ }^{25}$ I. Gorelov, ${ }^{105}$ B. Gorini, ${ }^{30}$ E. Gorini, ${ }^{73 a, 73 b}$ A. Gorišek, ${ }^{75}$ E. Gornicki, ${ }^{39}$ A. T. Goshaw, ${ }^{45}$ C. Gössling, ${ }^{43}$ M. I. Gostkin, ${ }^{65}$ D. Goujdami, ${ }^{135 c}$ A. G. Goussiou, ${ }^{138}$ N. Govender, ${ }^{145 b}$ E. Gozani, ${ }^{152}$ H. M. X. Grabas, ${ }^{137}$ L. Graber ${ }^{54}$ I. Grabowska-Boldd, ${ }^{38 a}$ P. Grafström, ${ }^{20 a, 20 b}$ K-J. Grahn, ${ }^{42}$ J. Gramling, ${ }^{49}$ E. Gramstad, ${ }^{119}$ S. Grancagnolo, ${ }^{16}$ V. Grassi, ${ }^{148}$ V. Gratchev, ${ }^{123}$ H. M. Gray, ${ }^{30}$ E. Graziani, ${ }^{134 a}$ Z. D. Greenwood, ${ }^{79,0}$ K. Gregersen, ${ }^{78}$ I. M. Gregor, ${ }^{42}$ P. Grenier, ${ }^{143}$ J. Griffiths, ${ }^{8}$ A. A. Grillo, ${ }^{137}$ K. Grimm, ${ }^{72}$ S. Grinstein, ${ }^{12, p}$ Ph. Gris, ${ }^{34}$ J.-F. Grivaz, ${ }^{117}$ J. P. Grohs,${ }^{44}$ A. Grohsjean, ${ }^{42}$ E. Gross,${ }^{172}$ J. Grosse-Knetter, ${ }^{54}$ G. C. Grossi ${ }^{79}$ Z. J. Grout, ${ }^{149}$ L. Guan, ${ }^{33 b}$ J. Guenther, ${ }^{128}$ F. Guescini, ${ }^{49}$ D. Guest,,${ }^{176}$ O. Gueta, ${ }^{153}$ E. Guido, ${ }^{50,50 b}$ T. Guillemin, ${ }^{117}$

S. Guindon, ${ }^{2}$ U. Gul, ${ }^{53}$ C. Gumpert, ${ }^{44}$ J. Guo, ${ }^{33 \mathrm{e}}$ Y. Guo, ${ }^{33 \mathrm{~b}}$ S. Gupta, ${ }^{120}$ G. Gustavino, ${ }^{132 a, 132 \mathrm{~b}}$ P. Gutierrez, ${ }^{113}$ N. G. Gutierrez Ortiz ${ }^{53}$ C. Gutschow, ${ }^{44}$ C. Guyot, ${ }^{136}$ C. Gwenlan, ${ }^{120}$ C. B. Gwilliam, ${ }^{74}$ A. Haas, ${ }^{110}$ C. Haber, ${ }^{15}$ H. K. Hadavand, ${ }^{8}$ N. Haddad, ${ }^{135 e}$ P. Haefner, ${ }^{21}$ S. Hageböck,${ }^{21}$ Z. Hajduk,${ }^{39}$ H. Hakobyan, ${ }^{177}$ M. Haleem, ${ }^{42}$ J. Haley, ${ }^{114}$ D. Hall, ${ }^{120}$ G. Halladjian, ${ }^{90}$ G. D. Hallewell,${ }^{85}$ K. Hamacher, ${ }^{175}$ P. Hamal, ${ }^{115}$ K. Hamano, ${ }^{169}$ M. Hamer, ${ }^{54}$ A. Hamilton, ${ }^{145 a}$ G. N. Hamity, ${ }^{145 c}$ P. G. Hamnett, ${ }^{42}$ L. Han,${ }^{33 b}$ K. Hanagaki, ${ }^{118}$ K. Hanawa,${ }^{155}$ M. Hance, ${ }^{15}$ P. Hanke, ${ }^{58 a}$ R. Hanna, ${ }^{136}$ J. B. Hansen, ${ }^{36}$ J. D. Hansen, ${ }^{36}$ M. C. Hansen, ${ }^{21}$ P. H. Hansen, ${ }^{36}$ K. Hara, ${ }^{160}$ A. S. Hard, ${ }^{173}$ T. Harenberg, ${ }^{175}$ F. Hariri, ${ }^{117}$ S. Harkusha, ${ }^{92}$ R. D. Harrington, ${ }^{46}$ P. F. Harrison, ${ }^{170}$ F. Hartjes, ${ }^{107}$ M. Hasegawa,${ }^{67}$ S. Hasegawa, ${ }^{103}$ Y. Hasegawa, ${ }^{140}$ A. Hasib, ${ }^{113}$ S. Hassani, ${ }^{136}$ S. Haug, ${ }^{17}$ R. Hauser, ${ }^{90}$ L. Hauswald, ${ }^{44}$ M. Havranek, ${ }^{127}$ C. M. Hawkes, ${ }^{18}$ R. J. Hawkings,${ }^{30}$ A. D. Hawkins, ${ }^{81}$ T. Hayashi, ${ }^{160}$ D. Hayden, ${ }^{90}$ C. P. Hays, ${ }^{120}$ J. M. Hays,${ }^{76}$ H. S. Hayward ${ }^{74}$ S. J. Haywood, ${ }^{131}$ S. J. Head, ${ }^{18}$ T. Heck, ${ }^{83}$ V. Hedberg, ${ }^{81}$ L. Heelan, ${ }^{8}$ S. Heim, ${ }^{122}$ T. Heim, ${ }^{175}$ B. Heinemann, ${ }^{15}$ L. Heinrich, ${ }^{110}$ J. Hejbal, ${ }^{127}$ L. Helary, ${ }^{22}$ S. Hellman, ${ }^{146 a, 146 b}$ D. Hellmich, ${ }^{21}$ C. Helsens,${ }^{30}$ J. Henderson, ${ }^{120}$ R. C. W. Henderson, ${ }^{72}$ Y. Heng, ${ }^{173}$ C. Hengler, ${ }^{42}$

A. Henrichs, ${ }^{176}$ A. M. Henriques Correia, ${ }^{30}$ S. Henrot-Versille, ${ }^{117}$ G. H. Herbert, ${ }^{16}$ Y. Hernández Jiménez, ${ }^{167}$ R. Herrberg-Schubert, ${ }^{16}$ G. Herten, ${ }^{48}$ R. Hertenberger, ${ }^{100}$ L. Hervas, ${ }^{30}$ G. G. Hesketh, ${ }^{78}$ N. P. Hessey, ${ }^{107}$ J. W. Hetherly, ${ }^{40}$ R. Hickling, ${ }^{76}$ E. Higón-Rodriguez, ${ }^{167}$ E. Hill, ${ }^{169}$ J. C. Hill, ${ }^{28}$ K. H. Hiller, ${ }^{42}$ S. J. Hillier, ${ }^{18}$ I. Hinchliffe, ${ }^{15}$ E. Hines, ${ }^{122}$ R. R. Hinman, ${ }^{15}$ M. Hirose, ${ }^{157}$ D. Hirschbuehl, ${ }^{175}$ J. Hobbs, ${ }^{148}$ N. Hod,${ }^{107}$ M. C. Hodgkinson,,${ }^{139}$ P. Hodgson, ${ }^{139}$ A. Hoecker, ${ }^{30}$ M. R. Hoeferkamp, ${ }^{105}$ F. Hoenig, ${ }^{100}$ M. Hohlfeld, ${ }^{83}$ D. Hohn, ${ }^{21}$ T. R. Holmes, ${ }^{15}$ M. Homann, ${ }^{43}$ T. M. Hong, ${ }^{125}$ L. Hooft van Huysduynen, ${ }^{110}$ W. H. Hopkins, ${ }^{116}$ Y. Horii, ${ }^{103}$ A. J. Horton, ${ }^{142}$ J-Y. Hostachy, ${ }^{55}$ S. Hou, ${ }^{151}$ A. Hoummada, ${ }^{135 a}$ 
J. Howard, ${ }^{120}$ J. Howarth, ${ }^{42}$ M. Hrabovsky, ${ }^{115}$ I. Hristova, ${ }^{16}$ J. Hrivnac, ${ }^{117}$ T. Hryn'ova, ${ }^{5}$ A. Hrynevich, ${ }^{93}$ C. Hsu, ${ }^{145 c}$ P. J. Hsu, ${ }^{151, q}$ S.-C. Hsu, ${ }^{138}$ D. Hu, ${ }^{35}$ Q. Hu,${ }^{33 b}$ X. Hu,${ }^{89}$ Y. Huang, ${ }^{42}$ Z. Hubacek,${ }^{30}$ F. Hubaut,${ }^{85}$ F. Huegging, ${ }^{21}$ T. B. Huffman, ${ }^{120}$ E. W. Hughes, ${ }^{35}$ G. Hughes, ${ }^{72}$ M. Huhtinen, ${ }^{30}$ T. A. Hülsing ${ }^{83}$ N. Huseynov, ${ }^{65, \mathrm{c}}$ J. Huston, ${ }^{90}$ J. Huth, ${ }^{57}$ G. Iacobucci, ${ }^{49}$ G. Iakovidis, ${ }^{25}$ I. Ibragimov, ${ }^{141}$ L. Iconomidou-Fayard, ${ }^{117}$ E. Ideal, ${ }^{176}$ Z. Idrissi, ${ }^{135 e}$ P. Iengo, ${ }^{30}$ O. Igonkina, ${ }^{107}$ T. Iizawa, ${ }^{171}$ Y. Ikegami, ${ }^{66}$ K. Ikematsu, ${ }^{141}$ M. Ikeno, ${ }^{66}$ Y. Ilchenko,,${ }^{31, \mathrm{r}}$ D. Iliadis, ${ }^{154} \mathrm{~N}$. Ilic, ${ }^{143}$ Y. Inamaru, ${ }^{67}$ T. Ince, ${ }^{101} \mathrm{G}$. Introzzi, ${ }^{121 \mathrm{a}, 121 \mathrm{~b}}$ P. Ioannou, ${ }^{9} \mathrm{M}$. Iodice, ${ }^{134 \mathrm{a}} \mathrm{K}$. Iordanidou, ${ }^{35} \mathrm{~V}$. Ippolito, ${ }^{57} \mathrm{~A}$. Irles Quiles, ${ }^{167} \mathrm{C}$. Isaksson, ${ }^{166}$ M. Ishino ${ }^{68} \mathrm{M}$. Ishitsuka, ${ }^{157} \mathrm{R}$. Ishmukhametov, ${ }^{111} \mathrm{C}$. Issever, ${ }^{120} \mathrm{~S}$. Istin, ${ }^{19 a} \mathrm{~J}$. M. Iturbe Ponce,${ }^{84} \mathrm{R}$. Iuppa, ${ }^{133 a, 133 \mathrm{~b}}$ J. Ivarsson, ${ }^{81}$ W. Iwanski, ${ }^{39}$ H. Iwasaki, ${ }^{66}$ J. M. Izen, ${ }^{41}$ V. Izzo, ${ }^{104 a}$ S. Jabbar, ${ }^{3}$ B. Jackson, ${ }^{122}$ M. Jackson,${ }^{74}$ P. Jackson, ${ }^{1}$ M. R. Jaekel,,${ }^{30}$ V. Jain, ${ }^{2}$ K. Jakobs, ${ }^{48}$ S. Jakobsen, ${ }^{30}$ T. Jakoubek, ${ }^{127}$ J. Jakubek, ${ }^{128}$ D. O. Jamin, ${ }^{114}$ D. K. Jana, ${ }^{79}$ E. Jansen, ${ }^{78}$ R. W. Jansky, ${ }^{62}$ J. Janssen, ${ }^{21}$ M. Janus, ${ }^{170}$ G. Jarlskog, ${ }^{81}$ N. Javadov, ${ }^{65, c}$ T. Javůrek,${ }^{48}$ L. Jeanty, ${ }^{15}$ J. Jejelava, ${ }^{51 a, s}$ G.-Y. Jeng, ${ }^{150}$ D. Jennens, ${ }^{88}$ P. Jenni, ${ }^{48, t}$ J. Jentzsch, ${ }^{43}$ C. Jeske, ${ }^{170}$ S. Jézéquel, ${ }^{5} \mathrm{H}$. Ji, ${ }^{173}$ J. Jia, ${ }^{148}$ Y. Jiang, ${ }^{33 b}$ S. Jiggins ${ }^{78}$

J. Jimenez Pena ${ }^{167}$ S. Jin, ${ }^{33 a}$ A. Jinaru, ${ }^{26 a}$ O. Jinnouchi, ${ }^{157}$ M. D. Joergensen ${ }^{36}$ P. Johansson, ${ }^{139}$ K. A. Johns, ${ }^{7}$ K. Jon-And, ${ }^{146 a, 146 b}$ G. Jones, ${ }^{170}$ R. W. L. Jones, ${ }^{72}$ T. J. Jones, ${ }^{74}$ J. Jongmanns, ${ }^{58 a}$ P. M. Jorge, ${ }^{126 a, 126 b}$ K. D. Joshi, ${ }^{84}$ J. Jovicevic, ${ }^{159 a}$ X. Ju, ${ }^{173}$ C. A. Jung, ${ }^{43}$ P. Jussel, ${ }^{62}$ A. Juste Rozas, ${ }^{12, p}$ M. Kaci, ${ }^{167}$ A. Kaczmarska, ${ }^{39}$ M. Kado, ${ }^{117}$ H. Kagan, ${ }^{111}$ M. Kagan, ${ }^{143}$ S. J. Kahn, ${ }^{85}$ E. Kajomovitz, ${ }^{45}$ C. W. Kalderon, ${ }^{120}$ S. Kama, ${ }^{40}$ A. Kamenshchikov, ${ }^{130}$ N. Kanaya, ${ }^{155}$ M. Kaneda ${ }^{30}$ S. Kaneti, ${ }^{28}$ V. A. Kantserov, ${ }^{98}$ J. Kanzaki, ${ }^{66}$ B. Kaplan, ${ }^{110}$ A. Kapliy, ${ }^{31}$ D. Kar, ${ }^{53}$ K. Karakostas, ${ }^{10}$ A. Karamaoun, ${ }^{3}$ N. Karastathis, ${ }^{10,107}$ M. J. Kareem, ${ }^{54}$ M. Karnevskiy, ${ }^{83}$ S. N. Karpov,${ }^{65}$ Z. M. Karpova ${ }^{65}$ K. Karthik, ${ }^{110}$ V. Kartvelishvili, ${ }^{72}$ A. N. Karyukhin, ${ }^{130}$ L. Kashif, ${ }^{173}$ R. D. Kass, ${ }^{111}$ A. Kastanas, ${ }^{14}$ Y. Kataoka, ${ }^{155}$ A. Katre, ${ }^{49}$ J. Katzy, ${ }^{42}$ K. Kawagoe, ${ }^{70}$ T. Kawamoto, ${ }^{155}$ G. Kawamura, ${ }^{54}$ S. Kazama, ${ }^{155}$ V. F. Kazanin, ${ }^{109, d}$ M. Y. Kazarinov, ${ }^{65}$ R. Keeler, ${ }^{169}$ R. Kehoe, ${ }^{40}$ J. S. Keller, ${ }^{42}$ J. J. Kempster, ${ }^{77}$ H. Keoshkerian, ${ }^{84}$ O. Kepka, ${ }^{127}$ B. P. Kerševan, ${ }^{75}$ S. Kersten, ${ }^{175}$ R. A. Keyes,${ }^{87}$ F. Khalil-zada, ${ }^{11}$ H. Khandanyan, ${ }^{146 a, 146 b}$ A. Khanov, ${ }^{114}$ A. G. Kharlamov, ${ }^{109, d}$ T. J. Khoo, ${ }^{28}$ V. Khovanskiy, ${ }^{97}$ E. Khramov, ${ }^{65}$ J. Khubua, ${ }^{51 b, u}$ H. Y. Kim ${ }^{8}$ H. Kim, ${ }^{146 a, 146 b}$ S. H. Kim, ${ }^{160}$ Y. Kim,${ }^{31}$ N. Kimura,${ }^{154}$ O. M. Kind, ${ }^{16}$ B. T. King ${ }^{74}$ M. King, ${ }^{167}$ S. B. King, ${ }^{168}$ J. Kirk, ${ }^{131}$ A. E. Kiryunin, ${ }^{101}$ T. Kishimoto, ${ }^{67}$ D. Kisielewska,${ }^{38 a}$ F. Kiss, ${ }^{48}$ K. Kiuchi, ${ }^{160}$ O. Kivernyk, ${ }^{136}$ E. Kladiva, ${ }^{144 b}$ M. H. Klein, ${ }^{35}$ M. Klein, ${ }^{74}$ U. Klein, ${ }^{74}$ K. Kleinknecht ${ }^{83}$ P. Klimek, ${ }^{146 a, 146 b}$ A. Klimentov, ${ }^{25}$ R. Klingenberg, ${ }^{43}$ J. A. Klinger, ${ }^{139}$ T. Klioutchnikova, ${ }^{30}$ E.-E. Kluge, ${ }^{58 a}$ P. Kluit, ${ }^{107}$ S. Kluth, ${ }^{101}$ E. Kneringer, ${ }^{62}$ E. B. F. G. Knoops ${ }^{85}$ A. Knue, ${ }^{53}$ A. Kobayashi, ${ }^{155}$ D. Kobayashi, ${ }^{157}$ T. Kobayashi, ${ }^{155}$ M. Kobel, ${ }^{44}$ M. Kocian, ${ }^{143}$ P. Kodys, ${ }^{129}$ T. Koffas, ${ }^{29}$ E. Koffeman, ${ }^{107}$ L. A. Kogan, ${ }^{120}$ S. Kohlmann, ${ }^{175}$ Z. Kohout,${ }^{128}$ T. Kohriki ${ }^{66}$ T. Koi, ${ }^{143}$ H. Kolanoski, ${ }^{16}$ I. Koletsou, ${ }^{5}$ A. A. Komar, ${ }^{96, a}$ Y. Komori, ${ }^{155}$ T. Kondo, ${ }^{66}$ N. Kondrashova, ${ }^{42}$ K. Köneke, ${ }^{48}$ A. C. König, ${ }^{106}$ S. König, ${ }^{83}$ T. Kono,${ }^{66, v}$ R. Konoplich, ${ }^{110, w}$ N. Konstantinidis, ${ }^{78}$ R. Kopeliansky, ${ }^{152}$ S. Koperny, ${ }^{38 a}$ L. Köpke, ${ }^{83}$ A. K. Kopp ${ }^{48}$ K. Korcyl, ${ }^{39}$ K. Kordas, ${ }^{154}$ A. Korn, ${ }^{78}$ A. A. Korol, ${ }^{109, d}$ I. Korolkov, ${ }^{12}$ E. V. Korolkova, ${ }^{139}$ O. Kortner, ${ }^{101}$ S. Kortner, ${ }^{101}$ T. Kosek, ${ }^{129}$ V. V. Kostyukhin, ${ }^{21}$ V. M. Kotov, ${ }^{65}$ A. Kotwal, ${ }^{45}$ A. Kourkoumeli-Charalampidi, ${ }^{154}$ C. Kourkoumelis, ${ }^{9}$ V. Kouskoura, ${ }^{25}$ A. Koutsman, ${ }^{159 a}$ R. Kowalewski, ${ }^{169}$ T. Z. Kowalski, ${ }^{38 a}$ W. Kozanecki, ${ }^{136}$ A. S. Kozhin, ${ }^{130}$ V. A. Kramarenko, ${ }^{99}$ G. Kramberger, ${ }^{75}$ D. Krasnopevtsev, ${ }^{98}$ M. W. Krasny, ${ }^{80}$ A. Krasznahorkay, ${ }^{30}$ J. K. Kraus, ${ }^{21}$ A. Kravchenko, ${ }^{25}$ S. Kreiss, ${ }^{110}$ M. Kretz, ${ }^{58 c}$ J. Kretzschmar,${ }^{74}$ K. Kreutzfeldt, ${ }^{52}$ P. Krieger, ${ }^{158}$ K. Krizka, ${ }^{31}$ K. Kroeninger, ${ }^{43}$ H. Kroha, ${ }^{101}$ J. Kroll, ${ }^{122}$ J. Kroseberg, ${ }^{21}$ J. Krstic, ${ }^{13}$ U. Kruchonak, ${ }^{65}$ H. Krüger, ${ }^{21}$ N. Krumnack, ${ }^{64}$ Z. V. Krumshteyn, ${ }^{65}$ A. Kruse, ${ }^{173}$ M. C. Kruse, ${ }^{45}$ M. Kruskal, ${ }^{22}$ T. Kubota, ${ }^{88}$ H. Kucuk, ${ }^{78}$ S. Kuday, ${ }^{4 b}$ S. Kuehn, ${ }^{48}$ A. Kugel, ${ }^{58 \mathrm{c}}$ F. Kuger, ${ }^{174}$ A. Kuhl, ${ }^{137}$ T. Kuhl, ${ }^{42}$ V. Kukhtin, ${ }^{65}$ Y. Kulchitsky, ${ }^{92}$ S. Kuleshov, ${ }^{32 \mathrm{~b}}$ M. Kuna, ${ }^{132 a, 132 \mathrm{~b}}$ T. Kunigo ${ }^{68}$ A. Kupco, ${ }^{127}$ H. Kurashige, ${ }^{67}$ Y. A. Kurochkin, ${ }^{92}$ R. Kurumida, ${ }^{67}$ V. Kus, ${ }^{127}$ E. S. Kuwertz, ${ }^{169}$ M. Kuze, ${ }^{157}$ J. Kvita, ${ }^{115}$ T. Kwan, ${ }^{169}$ D. Kyriazopoulos, ${ }^{139}$ A. La Rosa, ${ }^{49}$ J. L. La Rosa Navarro,${ }^{24 d}$ L. La Rotonda, ${ }^{37 a, 37 b}$ C. Lacasta, ${ }^{167}$ F. Lacava, ${ }^{132 a, 132 b}$ J. Lacey, ${ }^{29}$ H. Lacker, ${ }^{16}$ D. Lacour, ${ }^{80}$ V. R. Lacuesta, ${ }^{167}$ E. Ladygin, ${ }^{65}$ R. Lafaye, ${ }^{5}$ B. Laforge, ${ }^{80}$

T. Lagouri, ${ }^{176}$ S. Lai, ${ }^{48}$ L. Lambourne, ${ }^{78}$ S. Lammers, ${ }^{61}$ C. L. Lampen, ${ }^{7}$ W. Lampl, ${ }^{7}$ E. Lançon, ${ }^{136}$ U. Landgraf, ${ }^{48}$ M. P. J. Landon, ${ }^{76}$ V. S. Lang, ${ }^{58 a}$ J. C. Lange, ${ }^{12}$ A. J. Lankford, ${ }^{163}$ F. Lanni, ${ }^{25}$ K. Lantzsch ${ }^{30}$ A. Lanza, ${ }^{121 a}$ S. Laplace, ${ }^{80}$ C. Lapoire, ${ }^{30}$ J. F. Laporte, ${ }^{136}$ T. Lari, ${ }^{91 a}$ F. Lasagni Manghi, ${ }^{20 a}{ }^{20 b}$ M. Lassnig, ${ }^{30}$ P. Laurelli, ${ }^{47}$ W. Lavrijsen, ${ }^{15}$ A. T. Law, ${ }^{137}$ P. Laycock ${ }^{74}$ T. Lazovich, ${ }^{57}$ O. Le Dortz, ${ }^{80}$ E. Le Guirriec, ${ }^{85}$ E. Le Menedeu, ${ }^{12}$ M. LeBlanc, ${ }^{169}$ T. LeCompte, ${ }^{6}$ F. Ledroit-Guillon ${ }^{55}$ C. A. Lee ${ }^{145 b}$ S. C. Lee, ${ }^{151}$ L. Lee, ${ }^{1}$ G. Lefebvre ${ }^{80}$ M. Lefebvre, ${ }^{169}$ F. Legger, ${ }^{100}$ C. Leggett, ${ }^{15}$ A. Lehan, ${ }^{74}$ G. Lehmann Miotto, ${ }^{30}$ X. Lei, ${ }^{7}$ W. A. Leight ${ }^{29}$ A. Leisos, ${ }^{154, x}$ A. G. Leister, ${ }^{176}$ M. A. L. Leite, ${ }^{24 d}$ R. Leitner, ${ }^{129}$ D. Lellouch, ${ }^{172}$ B. Lemmer, ${ }^{54}$ K. J. C. Leney, ${ }^{78}$ T. Lenz,${ }^{21}$ B. Lenzi,${ }^{30}$ R. Leone, ${ }^{7}$ S. Leone, ${ }^{124 a, 124 b}$ C. Leonidopoulos ${ }^{46}$ S. Leontsinis, ${ }^{10}$ C. Leroy, ${ }^{95}$ C. G. Lester,${ }^{28}$ M. Levchenko, ${ }^{123}$ J. Levêque, ${ }^{5}$ D. Levin,${ }^{89}$ L. J. Levinson, ${ }^{172}$ M. Levy, ${ }^{18}$ A. Lewis, ${ }^{120}$ A. M. Leyko, ${ }^{21}$ M. Leyton, ${ }^{41}$ B. Li,${ }^{33 b, y}$ H. Li, ${ }^{148}$ H. L. Li,${ }^{31}$ L. Li,${ }^{45}$ L. Li $^{33 e}$ S. Li,${ }^{45}$ Y. Li ${ }^{33 c, z}$ Z. Liang, ${ }^{137}$ 
H. Liao, ${ }^{34}$ B. Liberti, ${ }^{133 a}$ A. Liblong ${ }^{158}$ P. Lichard ${ }^{30}$ K. Lie, ${ }^{165}$ J. Liebal, ${ }^{21}$ W. Liebig, ${ }^{14}$ C. Limbach,${ }^{21}$ A. Limosani, ${ }^{150}$ S. C. Lin, ${ }^{151, \text { aa }}$ T. H. Lin,${ }^{83}$ F. Linde,${ }^{107}$ B. E. Lindquist, ${ }^{148}$ J. T. Linnemann,${ }^{90}$ E. Lipeles, ${ }^{122}$ A. Lipniacka, ${ }^{14}$ M. Lisovyi,${ }^{58 b}$ T. M. Liss, ${ }^{165}$ D. Lissauer, ${ }^{25}$ A. Lister, ${ }^{168}$ A. M. Litke, ${ }^{137}$ B. Liu, ${ }^{151, b b}$ D. Liu, ${ }^{151}$ H. Liu, ${ }^{89}$ J. Liu, ${ }^{85}$ J. B. Liu, ${ }^{33 b}$ K. Liu, ${ }^{85}$ L. Liu, ${ }^{165}$ M. Liu, ${ }^{45}$ M. Liu, ${ }^{33 b}$ Y. Liu, ${ }^{33 b}$ M. Livan, ${ }^{121 a, 121 b}$ A. Lleres, ${ }^{55}$ J. Llorente Merino, ${ }^{82}$ S. L. Lloyd,${ }^{76}$ F. Lo Sterzo, ${ }^{151}$ E. Lobodzinska, ${ }^{42}$ P. Loch, ${ }^{7}$ W. S. Lockman, ${ }^{137}$ F. K. Loebinger, ${ }^{84}$ A. E. Loevschall-Jensen, ${ }^{36}$ A. Loginov,${ }^{176}$ T. Lohse, ${ }^{16}$ K. Lohwasser, ${ }^{42}$ M. Lokajicek, ${ }^{127}$ B. A. Long, ${ }^{22}$ J. D. Long ${ }^{89}$ R. E. Long, ${ }^{72}$ K. A. Looper, ${ }^{111}$ L. Lopes, ${ }^{126 a}$

D. Lopez Mateos ${ }^{57}$ B. Lopez Paredes ${ }^{139}$ I. Lopez Paz, ${ }^{12}$ J. Lorenz, ${ }^{100}$ N. Lorenzo Martinez,${ }^{61}$ M. Losada ${ }^{162}$ P. Loscutoff, ${ }^{15}$ P. J. Lösel,${ }^{100}$ X. Lou, ${ }^{33 a}$ A. Lounis, ${ }^{117}$ J. Love,${ }^{6}$ P. A. Love, ${ }^{72}$ N. Lu, ${ }^{89}$ H. J. Lubatti, ${ }^{138}$ C. Luci, ${ }^{132 a, 132 b}$ A. Lucotte,${ }^{55}$

F. Luehring, ${ }^{61}$ W. Lukas, ${ }^{62}$ L. Luminari, ${ }^{132 a}$ O. Lundberg, ${ }^{146 a, 146 b}$ B. Lund-Jensen, ${ }^{147}$ D. Lynn, ${ }^{25}$ R. Lysak, ${ }^{127}$ E. Lytken, ${ }^{81}$ H. Ma, ${ }^{25}$ L. L. Ma, ${ }^{33 \mathrm{~d}}$ G. Maccarrone, ${ }^{47}$ A. Macchiolo, ${ }^{101}$ C. M. Macdonald, ${ }^{139}$ J. Machado Miguens, ${ }^{122,126 b}$ D. Macina, ${ }^{30}$ D. Madaffari, ${ }^{85}$ R. Madar, ${ }^{34}$ H. J. Maddocks, ${ }^{72}$ W. F. Mader, ${ }^{44}$ A. Madsen, ${ }^{166}$ S. Maeland, ${ }^{14}$ T. Maeno, ${ }^{25}$ A. Maevskiy, ${ }^{99}$

E. Magradze ${ }^{54}$ K. Mahboubi, ${ }^{48}$ J. Mahlstedt, ${ }^{107}$ C. Maiani, ${ }^{136}$ C. Maidantchik, ${ }^{24 a}$ A. A. Maier, ${ }^{101}$ T. Maier, ${ }^{100}$ A. Maio, ${ }^{126 a, 126 b, 126 \mathrm{~d}}$ S. Majewski, ${ }^{116}$ Y. Makida, ${ }^{66}$ N. Makovec,${ }^{117}$ B. Malaescu, ${ }^{80}$ Pa. Malecki,${ }^{39}$ V. P. Maleev, ${ }^{123}$ F. Malek,${ }^{55}$ U. Mallik, ${ }^{63}$ D. Malon, ${ }^{6}$ C. Malone ${ }^{143}$ S. Maltezos, ${ }^{10}$ V. M. Malyshev, ${ }^{109}$ S. Malyukov, ${ }^{30}$ J. Mamuzic, ${ }^{42}$ G. Mancini, ${ }^{47}$ B. Mandelli, ${ }^{30}$ L. Mandelli, ${ }^{91 \mathrm{a}}$ I. Mandic,${ }^{75}$ R. Mandrysch, ${ }^{63}$ J. Maneira, ${ }^{126 a, 126 \mathrm{~b}}$ A. Manfredini, ${ }^{101}$

L. Manhaes de Andrade Filho, ${ }^{24 b}$ J. Manjarres Ramos, ${ }^{159 b}$ A. Mann, ${ }^{100}$ P. M. Manning, ${ }^{137}$ A. Manousakis-Katsikakis, ${ }^{9}$ B. Mansoulie, ${ }^{136}$ R. Mantifel,,${ }^{87}$ M. Mantoani ${ }^{54}$ L. Mapelli, ${ }^{30}$ L. March,${ }^{145 c}$ G. Marchiori,${ }^{80}$ M. Marcisovsky, ${ }^{127}$ C. P. Marino, ${ }^{169}$ M. Marjanovic, ${ }^{13}$ D. E. Marley,${ }^{89}$ F. Marroquim, ${ }^{24 a}$ S. P. Marsden,${ }^{84}$ Z. Marshall, ${ }^{15}$ L. F. Marti, ${ }^{17}$ S. Marti-Garcia, ${ }^{167}$ B. Martin, ${ }^{90}$ T. A. Martin, ${ }^{170}$ V. J. Martin, ${ }^{46}$ B. Martin dit Latour, ${ }^{14}$ M. Martinez, ${ }^{12, p}$ S. Martin-Haugh, ${ }^{131}$ V. S. Martoiu, ${ }^{26 a}$ A. C. Martyniuk, ${ }^{78}$ M. Marx,${ }^{138}$ F. Marzano, ${ }^{132 a}$ A. Marzin,${ }^{30}$ L. Masetti, ${ }^{83}$ T. Mashimo, ${ }^{155}$

R. Mashinistov, ${ }^{96}$ J. Masik, ${ }^{84}$ A. L. Maslennikov, ${ }^{109, \mathrm{~d}}$ I. Massa, ${ }^{20 \mathrm{a}, 20 \mathrm{~b}}$ L. Massa, ${ }^{20 \mathrm{a}, 20 \mathrm{~b}}$ N. Massol,${ }^{5}$ P. Mastrandrea, ${ }^{148}$ A. Mastroberardino,${ }^{37 a, 37 b}$ T. Masubuchi, ${ }^{155}$ P. Mättig, ${ }^{175}$ J. Mattmann, ${ }^{83}$ J. Maurer ${ }^{26 a}$ S. J. Maxfield, ${ }^{74}$ D. A. Maximov, ${ }^{109, d}$ R. Mazini, ${ }^{151}$ S. M. Mazza, ${ }^{91 a, 91 b}$ L. Mazzaferro, ${ }^{133 a, 133 b}$ G. Mc Goldrick, ${ }^{158}$ S. P. Mc Kee, ${ }^{89}$ A. McCarn, ${ }^{89}$ R. L. McCarthy, ${ }^{148}$ T. G. McCarthy, ${ }^{29}$ N. A. McCubbin, ${ }^{131}$ K. W. McFarlane, ${ }^{56, a}$ J. A. Mcfayden ${ }^{78}$ G. Mchedlidze ${ }^{54}$ S. J. McMahon, ${ }^{131}$ R. A. McPherson, ${ }^{169,1}$ M. Medinnis, ${ }^{42}$ S. Meehan, ${ }^{145 a}$ S. Mehlhase, ${ }^{100}$ A. Mehta, ${ }^{74}$ K. Meier, ${ }^{58 a}$ C. Meineck, ${ }^{100}$ B. Meirose, ${ }^{41}$ B. R. Mellado Garcia, ${ }^{145 \mathrm{c}}$ F. Meloni, ${ }^{17}$ A. Mengarelli, ${ }^{20 a, 20 b}$ S. Menke, ${ }^{101}$ E. Meoni, ${ }^{161}$ K. M. Mercurio, ${ }^{57}$ S. Mergelmeyer ${ }^{21}$ P. Mermod, ${ }^{49}$ L. Merola, ${ }^{104 a, 104 b}$ C. Meroni, ${ }^{91 a}$ F. S. Merritt,${ }^{31}$ A. Messina, ${ }^{132 a, 132 b}$ J. Metcalfe, ${ }^{25}$ A. S. Mete, ${ }^{163}$ C. Meyer, ${ }^{83}$ C. Meyer, ${ }^{122}$ J-P. Meyer, ${ }^{136}$ J. Meyer ${ }^{107}$ R. P. Middleton, ${ }^{131}$ S. Miglioranzi, ${ }^{164 a, 164 c}$ L. Mijović, ${ }^{21}$ G. Mikenberg, ${ }^{172}$ M. Mikestikova, ${ }^{127}$ M. Mikuž ${ }^{75}$ M. Milesi ${ }^{88}$ A. Milic, ${ }^{30}$ D. W. Miller, ${ }^{31}$ C. Mills, ${ }^{46}$ A. Milov, ${ }^{172}$ D. A. Milstead, ${ }^{146 a, 146 b}$ A. A. Minaenko, ${ }^{130}$ Y. Minami, ${ }^{155}$ I. A. Minashvili, ${ }^{65}$ A. I. Mincer, ${ }^{110}$ B. Mindur, ${ }^{38 a}$ M. Mineev, ${ }^{65}$ Y. Ming, ${ }^{173}$ L. M. Mir, ${ }^{12}$ T. Mitani, ${ }^{171}$ J. Mitrevski, ${ }^{100}$ V. A. Mitsou, ${ }^{167}$ A. Miucci, ${ }^{49}$ P. S. Miyagawa, ${ }^{139}$ J. U. Mjörnmark, ${ }^{81}$ T. Moa, ${ }^{146 a, 146 b}$ K. Mochizuki, ${ }^{85}$ S. Mohapatra, ${ }^{35}$ W. Mohr ${ }^{48}$ S. Molander, ${ }^{146 a, 146 b}$ R. Moles-Valls, ${ }^{167}$

K. Mönig, ${ }^{42}$ C. Monini, ${ }^{55}$ J. Monk ${ }^{36}$ E. Monnier ${ }^{85}$ J. Montejo Berlingen, ${ }^{12}$ F. Monticelli, ${ }^{71}$ S. Monzani, ${ }^{132 a, 132 b}$ R. W. Moore, ${ }^{3}$ N. Morange, ${ }^{117}$ D. Moreno, ${ }^{162}$ M. Moreno Llácer, ${ }^{54}$ P. Morettini, ${ }^{50 a}$ M. Morgenstern, ${ }^{44}$ M. Morii ${ }^{57}$ M. Morinaga, ${ }^{155}$ V. Morisbak, ${ }^{119}$ S. Moritz, ${ }^{83}$ A. K. Morley, ${ }^{147}$ G. Mornacchi, ${ }^{30}$ J. D. Morris, ${ }^{76}$ S. S. Mortensen, ${ }^{36}$ A. Morton, ${ }^{53}$ L. Morvaj, ${ }^{103}$ M. Mosidze, ${ }^{51 b}$ J. Moss, ${ }^{111}$ K. Motohashi, ${ }^{157}$ R. Mount,${ }^{143}$ E. Mountricha, ${ }^{25}$ S. V. Mouraviev, ${ }^{96, a}$ E. J. W. Moyse, ${ }^{86}$ S. Muanza, ${ }^{85}$ R. D. Mudd ${ }^{18}$ F. Mueller, ${ }^{101}$ J. Mueller, ${ }^{125}$ K. Mueller, ${ }^{21}$ R. S. P. Mueller, ${ }^{100}$ T. Mueller, ${ }^{28}$

D. Muenstermann, ${ }^{49}$ P. Mullen, ${ }^{53}$ G. A. Mullier, ${ }^{17}$ Y. Munwes, ${ }^{153}$ J. A. Murillo Quijada, ${ }^{18}$ W. J. Murray, ${ }^{170,131}$ H. Musheghyan,${ }^{54}$ E. Musto, ${ }^{152}$ A. G. Myagkov, ${ }^{130, c c}$ M. Myska, ${ }^{128}$ O. Nackenhorst,${ }^{54}$ J. Nadal,${ }^{54}$ K. Nagai, ${ }^{120}$ R. Nagai, ${ }^{157}$ Y. Nagai, ${ }^{85}$ K. Nagano, ${ }^{66}$ A. Nagarkar, ${ }^{111}$ Y. Nagasaka,${ }^{59}$ K. Nagata, ${ }^{160}$ M. Nagel, ${ }^{101}$ E. Nagy, ${ }^{85}$ A. M. Nairz, ${ }^{30}$ Y. Nakahama ${ }^{30}$ K. Nakamura, ${ }^{66}$ T. Nakamura, ${ }^{155}$ I. Nakano, ${ }^{112}$ H. Namasivayam, ${ }^{41}$ R. F. Naranjo Garcia, ${ }^{42}$ R. Narayan, ${ }^{31}$ T. Naumann, ${ }^{42}$ G. Navarro, ${ }^{162}$ R. Nayyar, ${ }^{7}$ H. A. Neal,${ }^{89}$ P. Yu. Nechaeva, ${ }^{96}$ T. J. Neep, ${ }^{84}$ P. D. Nef, ${ }^{143}$ A. Negri, ${ }^{121 a, 121 b}$ M. Negrini ${ }^{20 \mathrm{a}}$ S. Nektarijevic, ${ }^{106}$ C. Nellist, ${ }^{117}$ A. Nelson, ${ }^{163}$ S. Nemecek, ${ }^{127}$ P. Nemethy, ${ }^{110}$ A. A. Nepomuceno, ${ }^{24 a}$ M. Nessi ${ }^{30, \text { dd }}$ M. S. Neubauer, ${ }^{165}$ M. Neumann, ${ }^{175}$ R. M. Neves ${ }^{110}$ P. Nevski, ${ }^{25}$ P. R. Newman, ${ }^{18}$ D. H. Nguyen, ${ }^{6}$ R. B. Nickerson, ${ }^{120}$ R. Nicolaidou, ${ }^{136}$ B. Nicquevert ${ }^{30}$ J. Nielsen, ${ }^{137}$ N. Nikiforou ${ }^{35}$ A. Nikiforov, ${ }^{16}$ V. Nikolaenko, ${ }^{130, c c}$ I. Nikolic-Audit ${ }^{80}$ K. Nikolopoulos, ${ }^{18}$ J. K. Nilsen, ${ }^{119}$ P. Nilsson, ${ }^{25}$ Y. Ninomiya, ${ }^{155}$ A. Nisati, ${ }^{132 a}$ R. Nisius, ${ }^{101}$ T. Nobe, ${ }^{157}$ M. Nomachi, ${ }^{118}$ I. Nomidis, ${ }^{29}$ T. Nooney ${ }^{76}$ S. Norberg, ${ }^{113}$ M. Nordberg,${ }^{30}$ O. Novgorodova, ${ }^{44}$ S. Nowak, ${ }^{101}$ M. Nozaki, ${ }^{66}$ L. Nozka, ${ }^{115}$ K. Ntekas, ${ }^{10}$ G. Nunes Hanninger ${ }^{88}$ T. Nunnemann, ${ }^{100}$ E. Nurse,${ }^{78}$ F. Nuti, ${ }^{88}$ B. J. O'Brien ${ }^{46}$ F. O'grady, ${ }^{7}$ D. C. O’Neil, ${ }^{142}$ V. O'Shea, ${ }^{53}$ F. G. Oakham, ${ }^{29, e}$ H. Oberlack, ${ }^{101}$ T. Obermann, ${ }^{21}$ J. Ocariz, ${ }^{80}$ A. Ochi, ${ }^{67}$ I. Ochoa, ${ }^{78}$ 
J. P. Ochoa-Ricoux, ${ }^{32 a}$ S. Oda ${ }^{70}$ S. Odaka, ${ }^{66}$ H. Ogren, ${ }^{61}$ A. Oh, ${ }^{84}$ S. H. Oh, ${ }^{45}$ C. C. Ohm, ${ }^{15}$ H. Ohman, ${ }^{166}$ H. Oide, ${ }^{30}$ W. Okamura, ${ }^{118}$ H. Okawa, ${ }^{160}$ Y. Okumura, ${ }^{31}$ T. Okuyama, ${ }^{155}$ A. Olariu, ${ }^{26 a}$ S. A. Olivares Pino, ${ }^{46}$ D. Oliveira Damazio, ${ }^{25}$ E. Oliver Garcia, ${ }^{167}$ A. Olszewski, ${ }^{39}$ J. Olszowska, ${ }^{39}$ A. Onofre, ${ }^{126 a, 126 e}$ P. U. E. Onyisi, ${ }^{31, r}$ C. J. Oram, ${ }^{159 a}$ M. J. Oreglia, ${ }^{31}$ Y. Oren, ${ }^{153}$ D. Orestano, ${ }^{134 a, 134 b}$ N. Orlando, ${ }^{154}$ C. Oropeza Barrera, ${ }^{53}$ R. S. Orr, ${ }^{158}$ B. Osculati, ${ }^{50 a, 50 b}$ R. Ospanov, ${ }^{84}$ G. Otero y Garzon, ${ }^{27}$ H. Otono, ${ }^{70}$ M. Ouchrif, ${ }^{135 d}$ E. A. Ouellette, ${ }^{169}$ F. Ould-Saada, ${ }^{119}$ A. Ouraou, ${ }^{136}$ K. P. Oussoren, ${ }^{107}$ Q. Ouyang, ${ }^{33 a}$ A. Ovcharova, ${ }^{15}$ M. Owen,${ }^{53}$ R. E. Owen, ${ }^{18}$ V. E. Ozcan, ${ }^{19 a}$ N. Ozturk, ${ }^{8}$ K. Pachal, ${ }^{142}$ A. Pacheco Pages, ${ }^{12}$ C. Padilla Aranda ${ }^{12}$ M. Pagáčová, ${ }^{48}$ S. Pagan Griso, ${ }^{15}$ E. Paganis, ${ }^{139}$ C. Pahl,,${ }^{101}$ F. Paige,${ }^{25}$ P. Pais, ${ }^{86}$ K. Pajchel,${ }^{119}$ G. Palacino, ${ }^{159 \mathrm{~b}}$ S. Palestini, ${ }^{30}$ M. Palka, ${ }^{38 \mathrm{~b}}$ D. Pallin, ${ }^{34}$ A. Palma, ${ }^{126 a, 126 \mathrm{~b}}$ Y. B. Pan, ${ }^{173}$ E. Panagiotopoulou, ${ }^{10}$ C. E. Pandini, ${ }^{80}$ J. G. Panduro Vazquez,${ }^{77}$ P. Pani, ${ }^{146 a, 146 b}$ S. Panitkin, ${ }^{25}$ D. Pantea ${ }^{26 a}$ L. Paolozzi ${ }^{49}$ Th. D. Papadopoulou, ${ }^{10}$ K. Papageorgiou, ${ }^{154}$ A. Paramonov, ${ }^{6}$ D. Paredes Hernandez,${ }^{154}$ M. A. Parker, ${ }^{28}$ K. A. Parker, ${ }^{139}$ F. Parodi, ${ }^{50 a, 50 b}$ J. A. Parsons, ${ }^{35}$ U. Parzefall, ${ }^{48}$ E. Pasqualucci, ${ }^{132 a}$ S. Passaggio, ${ }^{50 a}$ F. Pastore, ${ }^{134 a, 134 b, a}$ Fr. Pastore ${ }^{77}$ G. Pásztor, ${ }^{29}$ S. Pataraia, ${ }^{175}$ N. D. Patel, ${ }^{150}$ J. R. Pater, ${ }^{84}$ T. Pauly, ${ }^{30}$ J. Pearce, ${ }^{169}$ B. Pearson, ${ }^{113}$ L. E. Pedersen, ${ }^{36}$ M. Pedersen, ${ }^{119}$ S. Pedraza Lopez,${ }^{167}$ R. Pedro, ${ }^{126 a, 126 b}$ S. V. Peleganchuk, ${ }^{109, d}$ D. Pelikan, ${ }^{166}$ O. Penc, ${ }^{127}$ H. Peng, ${ }^{33 b}$ B. Penning, ${ }^{31}$ J. Penwell, ${ }^{61}$ D. V. Perepelitsa, ${ }^{25}$ E. Perez Codina,${ }^{159 a}$ M. T. Pérez García-Estañ,${ }^{167}$ L. Perini, ${ }^{91 a, 91 b}$ H. Pernegger, ${ }^{30}$ S. Perrella, ${ }^{104 a, 104 b}$ R. Peschke, ${ }^{42}$ V. D. Peshekhonov, ${ }^{65}$ K. Peters, ${ }^{30}$ R. F. Y. Peters, ${ }^{84}$ B. A. Petersen, ${ }^{30}$ T. C. Petersen, ${ }^{36}$ E. Petit, ${ }^{42}$ A. Petridis, ${ }^{146 a, 146 \mathrm{~b}}$ C. Petridou, ${ }^{154}$ E. Petrolo, ${ }^{132 \mathrm{a}}$ F. Petrucci, ${ }^{134 a, 134 \mathrm{~b}}$ N. E. Pettersson, ${ }^{157}$ R. Pezoa, ${ }^{32 \mathrm{~b}}$ P. W. Phillips,${ }^{131}$ G. Piacquadio, ${ }^{143}$ E. Pianori, ${ }^{170}$ A. Picazio, ${ }^{49}$ E. Piccaro, ${ }^{76}$ M. Piccinini, ${ }^{20 a, 20 b}$ M. A. Pickering, ${ }^{120}$ R. Piegaia, ${ }^{27}$ D. T. Pignotti, ${ }^{111}$ J. E. Pilcher, ${ }^{31}$ A. D. Pilkington, ${ }^{84}$ J. Pina, ${ }^{126 a, 126 b, 126 d}$ M. Pinamonti, ${ }^{164 a, 164 c, e e}$ J. L. Pinfold, ${ }^{3}$ A. Pingel,${ }^{36}$ B. Pinto, ${ }^{126 a}$ S. Pires,${ }^{80}$ H. Pirumov, ${ }^{42}$ M. Pitt, ${ }^{172}$ C. Pizio, ${ }^{91 a, 91 b}$ L. Plazak, ${ }^{144 a}$ M.-A. Pleier,${ }^{25}$ V. Pleskot, ${ }^{129}$ E. Plotnikova, ${ }^{65}$ P. Plucinski, ${ }^{146 a, 146 b}$ D. Pluth, ${ }^{64}$ R. Poettgen, ${ }^{146 a, 146 b}$ L. Poggioli, ${ }^{117}$ D. Pohl, ${ }^{21}$ G. Polesello, ${ }^{121 a}$ A. Poley, ${ }^{42}$ A. Policicchio, ${ }^{37 a, 37 b}$ R. Polifka, ${ }^{158}$ A. Polini, ${ }^{20 a}$ C. S. Pollard, ${ }^{53}$ V. Polychronakos, ${ }^{25}$ K. Pommès, ${ }^{30}$ L. Pontecorvo, ${ }^{132 a}$ B. G. Pope,${ }^{90}$ G. A. Popeneciu, ${ }^{26 b}$ D. S. Popovic, ${ }^{13}$ A. Poppleton, ${ }^{30}$ S. Pospisil,${ }^{128}$ K. Potamianos, ${ }^{15}$ I. N. Potrap,${ }^{65}$

C. J. Potter, ${ }^{149}$ C. T. Potter,${ }^{16}$ G. Poulard, ${ }^{30}$ J. Poveda,${ }^{30}$ V. Pozdnyakov, ${ }^{65}$ P. Pralavorio, ${ }^{85}$ A. Pranko, ${ }^{15}$ S. Prasad, ${ }^{30}$ S. Prell,${ }^{64}$ D. Price, ${ }^{84}$ L. E. Price, ${ }^{6}$ M. Primavera ${ }^{73 a}$ S. Prince,${ }^{87}$ M. Proissl,${ }^{46}$ K. Prokofiev,${ }^{60 c}$ F. Prokoshin,${ }^{32 b}$ E. Protopapadaki, ${ }^{136}$ S. Protopopescu, ${ }^{25}$ J. Proudfoot ${ }^{6}$ M. Przybycien, ${ }^{38 a}$ E. Ptacek, ${ }^{116}$ D. Puddu, ${ }^{134 a, 134 b}$ E. Pueschel, ${ }^{86}$ D. Puldon, ${ }^{148}$ M. Purohit, ${ }^{25, f f}$ P. Puzo, ${ }^{117}$ J. Qian, ${ }^{89}$ G. Qin, ${ }^{53}$ Y. Qin,${ }^{84}$ A. Quadt, ${ }^{54}$ D. R. Quarrie, ${ }^{15}$ W. B. Quayle, ${ }^{164 a, 164 b}$ M. Queitsch-Maitland ${ }^{84}$ D. Quilty, ${ }^{53}$ S. Raddum, ${ }^{119}$ V. Radeka, ${ }^{25}$ V. Radescu, ${ }^{42}$ S. K. Radhakrishnan, ${ }^{148}$ P. Radloff, ${ }^{116}$ P. Rados ${ }^{88}$ F. Ragusa, ${ }^{91 a, 91 b}$ G. Rahal, ${ }^{178}$ S. Rajagopalan, ${ }^{25}$ M. Rammensee,${ }^{30}$ C. Rangel-Smith, ${ }^{166}$ F. Rauscher, ${ }^{100}$ S. Rave,${ }^{83}$ T. Ravenscroft, ${ }^{53}$ M. Raymond, ${ }^{30}$ A. L. Read, ${ }^{119}$ N. P. Readioff, ${ }^{74}$ D. M. Rebuzzi, ${ }^{121 a, 121 b}$ A. Redelbach, ${ }^{174}$ G. Redlinger,${ }^{25}$ R. Reece,${ }^{137}$ K. Reeves, ${ }^{41}$ L. Rehnisch, ${ }^{16}$ H. Reisin, ${ }^{27}$ M. Relich,${ }^{163}$ C. Rembser, ${ }^{30}$ H. Ren, ${ }^{33 a}$ A. Renaud, ${ }^{117}$ M. Rescigno, ${ }^{132 a}$ S. Resconi, ${ }^{91 \mathrm{a}}$ O. L. Rezanova, ${ }^{109, \mathrm{~d}}$ P. Reznicek, ${ }^{129}$ R. Rezvani, ${ }^{95}$ R. Richter,${ }^{101}$ S. Richter, ${ }^{78}$ E. Richter-Was,${ }^{38 b}$ O. Ricken, ${ }^{21}$ M. Ridel,${ }^{80}$ P. Rieck, ${ }^{16}$ C. J. Riegel,${ }^{175}$ J. Rieger ${ }^{54}$ M. Rijssenbeek, ${ }^{148}$ A. Rimoldi, ${ }^{121 a, 121 b}$ L. Rinaldi, ${ }^{20 a}$ B. Ristić, ${ }^{49}$

E. Ritsch, ${ }^{30}$ I. Riu, ${ }^{12}$ F. Rizatdinova, ${ }^{114}$ E. Rizvi, ${ }^{76}$ S. H. Robertson, ${ }^{87,1}$ A. Robichaud-Veronneau, ${ }^{87}$ D. Robinson, ${ }^{28}$ J. E. M. Robinson, ${ }^{84}$ A. Robson, ${ }^{53}$ C. Roda,${ }^{124 a, 124 b}$ S. Roe, ${ }^{30}$ O. Røhne, ${ }^{119}$ S. Rolli, ${ }^{161}$ A. Romaniouk,${ }^{98}$ M. Romano ${ }^{20 a, 20 b}$ S. M. Romano Saez, ${ }^{34}$ E. Romero Adam, ${ }^{167}$ N. Rompotis, ${ }^{138}$ M. Ronzani, ${ }^{48}$ L. Roos,${ }^{80}$ E. Ros, ${ }^{167}$ S. Rosati, ${ }^{132 a}$ K. Rosbach, ${ }^{48}$ P. Rose, ${ }^{137}$ P. L. Rosendahl,${ }^{14}$ O. Rosenthal, ${ }^{141}$ V. Rossetti, ${ }^{146 a, 146 b}$ E. Rossi, ${ }^{104 a, 104 b}$ L. P. Rossi, ${ }^{50 a}$ R. Rosten, ${ }^{138}$ M. Rotaru, ${ }^{26 a}$ I. Roth, ${ }^{172}$ J. Rothberg, ${ }^{138}$ D. Rousseau, ${ }^{17}$ C. R. Royon, ${ }^{136}$ A. Rozanov, ${ }^{85}$ Y. Rozen, ${ }^{152}$ X. Ruan, ${ }^{145}$ F. Rubbo, ${ }^{143}$ I. Rubinskiy, ${ }^{42}$ V. I. Rud, ${ }^{99}$ C. Rudolph, ${ }^{44}$ M. S. Rudolph ${ }^{158}$ F. Rühr, ${ }^{48}$ A. Ruiz-Martinez, ${ }^{30}$ Z. Rurikova, ${ }^{48}$ N. A. Rusakovich, ${ }^{65}$ A. Ruschke, ${ }^{100}$ H. L. Russell, ${ }^{138}$ J. P. Rutherfoord, ${ }^{7}$ N. Ruthmann, ${ }^{48}$ Y. F. Ryabov, ${ }^{123}$ M. Rybar, ${ }^{129}$ G. Rybkin, ${ }^{117}$ N. C. Ryder, ${ }^{120}$ A. F. Saavedra,${ }^{150}$ G. Sabato ${ }^{107}$ S. Sacerdoti, ${ }^{27}$ A. Saddique, ${ }^{3}$

H. F-W. Sadrozinski, ${ }^{137}$ R. Sadykov,${ }^{65}$ F. Safai Tehrani, ${ }^{132 a}$ M. Saimpert, ${ }^{136}$ H. Sakamoto, ${ }^{155}$ Y. Sakurai, ${ }^{171}$ G. Salamanna, ${ }^{134 a, 134 b}$ A. Salamon, ${ }^{133 a}$ M. Saleem, ${ }^{113}$ D. Salek, ${ }^{107}$ P. H. Sales De Bruin, ${ }^{138}$ D. Salihagic, ${ }^{101}$ A. Salnikov, ${ }^{143}$ J. Salt,${ }^{167}$ D. Salvatore, ${ }^{37 a, 37 b}$ F. Salvatore, ${ }^{149}$ A. Salvucci, ${ }^{106}$ A. Salzburger, ${ }^{30}$ D. Sampsonidis, ${ }^{154}$ A. Sanchez, ${ }^{104 a, 104 b}$ J. Sánchez, ${ }^{167}$ V. Sanchez Martinez, ${ }^{167}$ H. Sandaker, ${ }^{14}$ R. L. Sandbach,${ }^{76}$ H. G. Sander, ${ }^{83}$ M. P. Sanders, ${ }^{100}$ M. Sandhoff, ${ }^{175}$ C. Sandoval, ${ }^{162}$ R. Sandstroem, ${ }^{101}$ D. P. C. Sankey, ${ }^{131}$ M. Sannino, ${ }^{50 a, 50 b}$ A. Sansoni, ${ }^{47}$ C. Santoni,${ }^{34}$ R. Santonico, ${ }^{133 a, 133 b}$ H. Santos, ${ }^{126 a}$ I. Santoyo Castillo, ${ }^{149}$ K. Sapp, ${ }^{125}$ A. Sapronov, ${ }^{65}$ J. G. Saraiva, ${ }^{126 a, 126 d}$ B. Sarrazin, ${ }^{21}$ O. Sasaki, ${ }^{66}$ Y. Sasaki, ${ }^{155}$ K. Sato, ${ }^{160}$ G. Sauvage, ${ }^{5, a}$ E. Sauvan, ${ }^{5}$ G. Savage,${ }^{77}$ P. Savard, ${ }^{158, e}$ C. Sawyer,${ }^{131}$ L. Sawyer, ${ }^{79,0}$ J. Saxon, ${ }^{31}$ C. Sbarra, ${ }^{20 \mathrm{a}}$ A. Sbrizzi, ${ }^{20 a, 20 \mathrm{~b}}$ T. Scanlon, ${ }^{78}$ D. A. Scannicchio, ${ }^{163}$ M. Scarcella, ${ }^{150}$ V. Scarfone, ${ }^{37 \mathrm{a}, 37 \mathrm{~b}}$ J. Schaarschmidt, ${ }^{172}$ P. Schacht, ${ }^{101}$ D. Schaefer, ${ }^{30}$ R. Schaefer, ${ }^{42}$ J. Schaeffer, ${ }^{83}$ S. Schaepe, ${ }^{21}$ S. Schaetzel, ${ }^{58 b}$ U. Schäfer, ${ }^{83}$ A. C. Schaffer, ${ }^{117}$ 
D. Schaile, ${ }^{100}$ R. D. Schamberger, ${ }^{148}$ V. Scharf, ${ }^{58 a}$ V. A. Schegelsky, ${ }^{123}$ D. Scheirich, ${ }^{129}$ M. Schernau, ${ }^{163}$ C. Schiavi, ${ }^{50 a, 50 b}$ C. Schillo, ${ }^{48}$ M. Schioppa, ${ }^{37 a, 37 b}$ S. Schlenker, ${ }^{30}$ E. Schmidt, ${ }^{48}$ K. Schmieden, ${ }^{30}$ C. Schmitt, ${ }^{83}$ S. Schmitt, ${ }^{58 b}$ S. Schmitt, ${ }^{42}$ B. Schneider, ${ }^{159 a}$ Y. J. Schnellbach, ${ }^{74}$ U. Schnoor, ${ }^{44}$ L. Schoeffel, ${ }^{136}$ A. Schoening, ${ }^{58 b}$ B. D. Schoenrock, ${ }^{90}$ E. Schopf, ${ }^{21}$ A. L. S. Schorlemmer, ${ }^{54}$ M. Schott, ${ }^{83}$ D. Schouten, ${ }^{159 a}$ J. Schovancova, ${ }^{8}$ S. Schramm, ${ }^{158}$ M. Schreyer, ${ }^{174}$ C. Schroeder, ${ }^{83}$ N. Schuh, ${ }^{83}$ M. J. Schultens, ${ }^{21}$ H.-C. Schultz-Coulon, ${ }^{58 a}$ H. Schulz,${ }^{16}$ M. Schumacher, ${ }^{48}$ B. A. Schumm, ${ }^{137}$ Ph. Schune, ${ }^{136}$ C. Schwanenberger ${ }^{84}$ A. Schwartzman, ${ }^{143}$ T. A. Schwarz, ${ }^{89}$ Ph. Schwegler, ${ }^{101}$ Ph. Schwemling, ${ }^{136}$ R. Schwienhorst, ${ }^{90}$ J. Schwindling, ${ }^{136}$ T. Schwindt, ${ }^{21}$ F. G. Sciacca, ${ }^{17}$ E. Scifo, ${ }^{117}$ G. Sciolla, ${ }^{23}$ F. Scuri, ${ }^{124 a, 124 b}$ F. Scutti, ${ }^{21}$ J. Searcy, ${ }^{89}$ G. Sedov, ${ }^{42}$ E. Sedykh, ${ }^{123}$ P. Seema, ${ }^{21}$ S. C. Seidel, ${ }^{105}$ A. Seiden, ${ }^{137}$ F. Seifert, ${ }^{128}$ J. M. Seixas, ${ }^{24 a}$ G. Sekhniaidze, ${ }^{104 a}$ K. Sekhon, ${ }^{89}$ S. J. Sekula, ${ }^{40}$ D. M. Seliverstov, ${ }^{123, a}$ N. Semprini-Cesari, ${ }^{20 a, 20 b}$ C. Serfon ${ }^{30}$ L. Serin, ${ }^{117}$ L. Serkin, ${ }^{164 a, 164 b}$ T. Serre, ${ }^{85}$ M. Sessa, ${ }^{134 a, 134 b}$ R. Seuster, ${ }^{159 a}$ H. Severini, ${ }^{113}$ T. Sfiligoj,${ }^{75}$ F. Sforza, ${ }^{30}$ A. Sfyrla, ${ }^{30}$ E. Shabalina, ${ }^{54}$ M. Shamim, ${ }^{116}$ L. Y. Shan, ${ }^{33 a}$ R. Shang, ${ }^{165}$ J. T. Shank,${ }^{22}$ M. Shapiro, ${ }^{15}$ P. B. Shatalov, ${ }^{97}$ K. Shaw, ${ }^{164 a, 164 b}$ S. M. Shaw ${ }^{84}$ A. Shcherbakova, ${ }^{146 a, 146 b}$ C. Y. Shehu, ${ }^{149}$ P. Sherwood, ${ }^{78}$ L. Shi, ${ }^{151, g g}$ S. Shimizu, ${ }^{67}$ C. O. Shimmin, ${ }^{163}$ M. Shimojima, ${ }^{102}$ M. Shiyakova, ${ }^{65}$ A. Shmeleva, ${ }^{96}$ D. Shoaleh Saadi, ${ }^{95}$ M. J. Shochet, ${ }^{31}$ S. Shojaii, ${ }^{11 a, 91 b}$ S. Shrestha, ${ }^{111}$ E. Shulga, ${ }^{98}$ M. A. Shupe, ${ }^{7}$ S. Shushkevich ${ }^{42}$ P. Sicho, ${ }^{127}$ O. Sidiropoulou, ${ }^{174}$ D. Sidorov, ${ }^{114}$ A. Sidoti, ${ }^{20 a, 20 b}$ F. Siegert, ${ }^{44}$ Dj. Sijacki, ${ }^{13}$ J. Silva, ${ }^{126 a, 126 d}$ Y. Silver, ${ }^{153}$ S. B. Silverstein, ${ }^{146 a}$ V. Simak, ${ }^{128}$ O. Simard, ${ }^{5}$ Lj. Simic, ${ }^{13}$ S. Simion, ${ }^{117}$ E. Simioni ${ }^{83}$ B. Simmons, ${ }^{78}$ D. Simon, ${ }^{34}$ R. Simoniello, ${ }^{91 \mathrm{a}, 91 \mathrm{~b}}$ P. Sinervo, ${ }^{158}$ N. B. Sinev, ${ }^{116}$ G. Siragusa, ${ }^{174}$ A. N. Sisakyan, ${ }^{65, \mathrm{a}}$ S. Yu. Sivoklokov, ${ }^{99}$ J. Sjölin, ${ }^{146 a, 146 b}$ T. B. Sjursen, ${ }^{14}$ M. B. Skinner, ${ }^{72}$ H. P. Skottowe, ${ }^{57}$ P. Skubic, ${ }^{113}$ M. Slater, ${ }^{18}$ T. Slavicek, ${ }^{128}$ M. Slawinska, ${ }^{107}$ K. Sliwa, ${ }^{161}$ V. Smakhtin, ${ }^{172}$ B. H. Smart, ${ }^{46}$ L. Smestad, ${ }^{14}$ S. Yu. Smirnov, ${ }^{98}$ Y. Smirnov,${ }^{98}$ L. N. Smirnova, ${ }^{99, \text { hh }}$ O. Smirnova, ${ }^{81}$ M. N. K. Smith ${ }^{35}$ R. W. Smith,${ }^{35}$ M. Smizanska, ${ }^{72}$ K. Smolek, ${ }^{128}$ A. A. Snesarev, ${ }^{96}$ G. Snidero, ${ }^{76}$ S. Snyder, ${ }^{25}$ R. Sobie, ${ }^{169,1}$ F. Socher ${ }^{44}$ A. Soffer, ${ }^{153}$ D. A. Soh, ${ }^{151, g g}$ C. A. Solans,${ }^{30}$ M. Solar, ${ }^{128}$ J. Solc, ${ }^{128}$ E. Yu. Soldatov, ${ }^{98}$ U. Soldevila, ${ }^{167}$ A. A. Solodkov, ${ }^{130}$ A. Soloshenko, ${ }^{65}$ O. V. Solovyanov, ${ }^{130}$ V. Solovyev, ${ }^{123}$ P. Sommer, ${ }^{48}$ H. Y. Song, ${ }^{33 \mathrm{~b}}$ N. Soni, ${ }^{1}$ A. Sood,${ }^{15}$ A. Sopczak, ${ }^{128}$ B. Sopko, ${ }^{128}$ V. Sopko, ${ }^{128}$ V. Sorin, ${ }^{12}$ D. Sosa, ${ }^{58 b}$ M. Sosebee, ${ }^{8}$ C. L. Sotiropoulou, ${ }^{124 a, 124 b}$ R. Soualah, ${ }^{164 a, 164 c}$ A. M. Soukharev, ${ }^{109, d}$ D. South, ${ }^{42}$ B. C. Sowden, ${ }^{77}$ S. Spagnolo, ${ }^{73 a, 73 b}$ M. Spalla, ${ }^{124 a, 124 b}$ F. Spanò, ${ }^{77}$ W. R. Spearman,${ }^{57}$ F. Spettel, ${ }^{101}$ R. Spighi, ${ }^{20 a}$ G. Spigo, ${ }^{30}$ L. A. Spiller ${ }^{88}$ M. Spousta, ${ }^{129}$ T. Spreitzer, ${ }^{158}$ R. D. St. Denis, ${ }^{53, a}$ S. Staerz, ${ }^{44}$ J. Stahlman, ${ }^{122}$ R. Stamen, ${ }^{58 a}$ S. Stamm, ${ }^{16}$ E. Stanecka ${ }^{39}$ C. Stanescu, ${ }^{134 a}$ M. Stanescu-Bellu, ${ }^{42}$ M. M. Stanitzki, ${ }^{42}$ S. Stapnes, ${ }^{119}$ E. A. Starchenko, ${ }^{130}$ J. Stark, ${ }^{55}$ P. Staroba, ${ }^{127}$ P. Starovoitov, ${ }^{42}$ R. Staszewski, ${ }^{39}$ P. Stavina, ${ }^{144 a, a}$ P. Steinberg, ${ }^{25}$ B. Stelzer, ${ }^{142}$ H. J. Stelzer, ${ }^{30}$ O. Stelzer-Chilton, ${ }^{159 a}$ H. Stenzel,${ }^{52}$ S. Stern, ${ }^{101}$ G. A. Stewart, ${ }^{53}$ J. A. Stillings,${ }^{21}$ M. C. Stockton, ${ }^{87}$ M. Stoebe, ${ }^{87}$ G. Stoicea ${ }^{26 a}$ P. Stolte, ${ }^{54}$ S. Stonjek, ${ }^{101}$ A. R. Stradling, ${ }^{8}$ A. Straessner, ${ }^{44}$ M. E. Stramaglia, ${ }^{17}$ J. Strandberg, ${ }^{147}$ S. Strandberg, ${ }^{146 a, 146 b}$ A. Strandlie, ${ }^{119}$ E. Strauss, ${ }^{143}$ M. Strauss, ${ }^{113}$ P. Strizenec, ${ }^{14 \mathrm{~b}}$ R. Ströhmer, ${ }^{174}$ D. M. Strom, ${ }^{116}$ R. Stroynowski, ${ }^{40}$ A. Strubig, ${ }^{106}$ S. A. Stucci, ${ }^{17}$ B. Stugu, ${ }^{14}$ N. A. Styles, ${ }^{42}$ D. Su, ${ }^{143}$ J. Su, ${ }^{125}$ R. Subramaniam, ${ }^{79}$ A. Succurro, ${ }^{12}$ Y. Sugaya, ${ }^{118}$ C. Suhr, ${ }^{108}$ M. Suk, ${ }^{128}$ V. V. Sulin, ${ }^{96}$ S. Sultansoy, ${ }^{4 \mathrm{c}}$ T. Sumida, ${ }^{68}$ S. Sun,${ }^{57}$ X. Sun, ${ }^{33 a}$ J. E. Sundermann, ${ }^{48}$ K. Suruliz, ${ }^{149}$ G. Susinno, ${ }^{37 a, 37 b}$ M. R. Sutton, ${ }^{149}$ S. Suzuki, ${ }^{66}$ Y. Suzuki, ${ }^{66}$ M. Svatos, ${ }^{127}$ S. Swedish,${ }^{168}$ M. Swiatlowski, ${ }^{143}$ I. Sykora, ${ }^{144 a}$ T. Sykora,${ }^{129}$ D. Ta, ${ }^{90}$ C. Taccini, ${ }^{134 a, 134 b}$ K. Tackmann, ${ }^{42}$ J. Taenzer, ${ }^{158}$ A. Taffard ${ }^{163}$ R. Tafirout, ${ }^{159 a}$ N. Taiblum, ${ }^{153}$ H. Takai, ${ }^{25}$ R. Takashima,${ }^{69}$ H. Takeda,${ }^{67}$ T. Takeshita, ${ }^{140}$ Y. Takubo, ${ }^{66}$ M. Talby, ${ }^{85}$ A. A. Talyshev, ${ }^{109, d}$ J. Y. C. Tam,${ }^{174}$ K. G. Tan, ${ }^{88}$ J. Tanaka, ${ }^{155}$ R. Tanaka, ${ }^{117}$ S. Tanaka, ${ }^{66}$ B. B. Tannenwald ${ }^{111}$ N. Tannoury, ${ }^{21}$ S. Tapprogge, ${ }^{83}$ S. Tarem, ${ }^{152}$ F. Tarrade, ${ }^{29}$ G. F. Tartarelli, ${ }^{91 a}$ P. Tas, ${ }^{129}$ M. Tasevsky, ${ }^{127}$ T. Tashiro, ${ }^{68}$ E. Tassi, ${ }^{37 a, 37 b}$ A. Tavares Delgado, ${ }^{126 a, 126 b}$ Y. Tayalati, ${ }^{135 d}$ F. E. Taylor, ${ }^{94}$ G. N. Taylor, ${ }^{88}$ W. Taylor, ${ }^{159 b}$ F. A. Teischinger, ${ }^{30}$ M. Teixeira Dias Castanheira, ${ }^{76}$ P. Teixeira-Dias, ${ }^{77}$ K. K. Temming, ${ }^{48}$ H. Ten Kate ${ }^{30}$ P. K. Teng, ${ }^{151}$ J. J. Teoh, ${ }^{118}$ F. Tepel, ${ }^{175}$ S. Terada,${ }^{66}$ K. Terashi, ${ }^{155}$ J. Terron, ${ }^{82}$ S. Terzo, ${ }^{101}$ M. Testa,${ }^{47}$ R. J. Teuscher, ${ }^{158,1}$ J. Therhaag, ${ }^{21}$ T. Theveneaux-Pelzer, ${ }^{34}$ J. P. Thomas,${ }^{18}$ J. Thomas-Wilsker, ${ }^{77}$ E. N. Thompson, ${ }^{35}$ P. D. Thompson, ${ }^{18}$ R. J. Thompson, ${ }^{84}$ A. S. Thompson, ${ }^{53}$ L. A. Thomsen, ${ }^{176}$ E. Thomson, ${ }^{122}$ M. Thomson, ${ }^{28}$ R. P. Thun, ${ }^{89, a}$ M. J. Tibbetts, ${ }^{15}$ R. E. Ticse Torres, ${ }^{85}$ V. O. Tikhomirov, ${ }^{96, i i}$ Yu. A. Tikhonov, ${ }^{109, \mathrm{~d}}$ S. Timoshenko, ${ }^{98}$ E. Tiouchichine,${ }^{85}$ P. Tipton, ${ }^{176}$ S. Tisserant, ${ }^{85}$ T. Todorov, ${ }^{5, a}$ S. Todorova-Nova, ${ }^{129}$ J. Tojo, ${ }^{70}$ S. Tokár, ${ }^{144 a}$ K. Tokushuku ${ }^{66}$ K. Tollefson, ${ }^{90}$ E. Tolley ${ }^{57}$ L. Tomlinson, ${ }^{84}$ M. Tomoto, ${ }^{103}$ L. Tompkins, ${ }^{143, j \mathrm{j}} \mathrm{K}$. Toms, ${ }^{105}$ E. Torrence, ${ }^{116}$ H. Torres,${ }^{142}$ E. Torró Pastor, ${ }^{167}$ J. Toth,${ }^{85, k k}$ F. Touchard, ${ }^{85}$ D. R. Tovey, ${ }^{139}$ T. Trefzger,,${ }^{174}$ L. Tremblet, ${ }^{30}$ A. Tricoli, ${ }^{30}$ I. M. Trigger, ${ }^{159 a}$ S. Trincaz-Duvoid,${ }^{80}$ M. F. Tripiana, ${ }^{12}$ W. Trischuk, ${ }^{158}$ B. Trocmé,${ }^{55}$ C. Troncon, ${ }^{91 a}$ M. Trottier-McDonald, ${ }^{15}$ M. Trovatelli, ${ }^{169}$ P. True, ${ }^{90}$ L. Truong, ${ }^{164 a, 164 \mathrm{c}}$ M. Trzebinski, ${ }^{39}$ A. Trzupek, ${ }^{39}$ C. Tsarouchas, ${ }^{30}$ J. C-L. Tseng, ${ }^{120}$ P. V. Tsiareshka,${ }^{92}$ D. Tsionou, ${ }^{154}$ G. Tsipolitis, ${ }^{10}$ N. Tsirintanis, ${ }^{9}$ S. Tsiskaridze,${ }^{12}$ V. Tsiskaridze, ${ }^{48}$ E. G. Tskhadadze, ${ }^{51 a}$ I. I. Tsukerman, ${ }^{97}$ V. Tsulaia, ${ }^{15}$ S. Tsuno, ${ }^{66}$ D. Tsybychev, ${ }^{148}$ A. Tudorache, ${ }^{26 \mathrm{a}}$ V. Tudorache, ${ }^{26 \mathrm{a}}$ A. N. Tuna, ${ }^{122}$ S. A. Tupputi, ${ }^{20 \mathrm{a}, 20 \mathrm{~b}}$ S. Turchikhin, ${ }^{99, \mathrm{hh}}$ 
D. Turecek, ${ }^{128}$ R. Turra,${ }^{91 a, 91 b}$ A. J. Turvey, ${ }^{40}$ P. M. Tuts, ${ }^{35}$ A. Tykhonov, ${ }^{49}$ M. Tylmad, ${ }^{146 a, 146 b}$ M. Tyndel, ${ }^{131}$ I. Ueda, ${ }^{155}$ R. Ueno, ${ }^{29}$ M. Ughetto, ${ }^{146 a, 146 b}$ M. Ugland, ${ }^{14}$ M. Uhlenbrock, ${ }^{21}$ F. Ukegawa, ${ }^{160}$ G. Unal,${ }^{30}$ A. Undrus, ${ }^{25}$ G. Unel, ${ }^{163}$ F. C. Ungaro, ${ }^{48}$ Y. Unno, ${ }^{66}$ C. Unverdorben, ${ }^{100}$ J. Urban, ${ }^{144 b}$ P. Urquijo, ${ }^{88}$ P. Urrejola, ${ }^{83}$ G. Usai, ${ }^{8}$ A. Usanova, ${ }^{62}$ L. Vacavant, ${ }^{85}$ V. Vacek, ${ }^{128}$ B. Vachon,${ }^{87}$ C. Valderanis, ${ }^{83}$ N. Valencic, ${ }^{107}$ S. Valentinetti, ${ }^{20 a, 20 b}$ A. Valero, ${ }^{167}$ L. Valery, ${ }^{12}$ S. Valkar, ${ }^{129}$ E. Valladolid Gallego, ${ }^{167}$ S. Vallecorsa ${ }^{49}$ J. A. Valls Ferrer, ${ }^{167}$ W. Van Den Wollenberg, ${ }^{107}$

P. C. Van Der Deijl ${ }^{107}$ R. van der Geer, ${ }^{107}$ H. van der Graaf, ${ }^{107}$ R. Van Der Leeuw, ${ }^{107}$ N. van Eldik, ${ }^{152}$ P. van Gemmeren, ${ }^{6}$ J. Van Nieuwkoop, ${ }^{142}$ I. van Vulpen, ${ }^{107}$ M. C. van Woerden, ${ }^{30}$ M. Vanadia, ${ }^{132 a, 132 b}$ W. Vandelli, ${ }^{30}$ R. Vanguri, ${ }^{122}$ A. Vaniachine, ${ }^{6}$ F. Vannucci, ${ }^{80}$ G. Vardanyan, ${ }^{177}$ R. Vari, ${ }^{132 a}$ E. W. Varnes, ${ }^{7}$ T. Varol, ${ }^{40}$ D. Varouchas, ${ }^{80}$ A. Vartapetian, ${ }^{8}$ K. E. Varvell, ${ }^{150}$ F. Vazeille, ${ }^{34}$ T. Vazquez Schroeder,${ }^{87}$ J. Veatch, ${ }^{7}$ L. M. Veloce, ${ }^{158}$ F. Veloso, ${ }^{126 a, 126 c}$ T. Velz, ${ }^{21}$ S. Veneziano, ${ }^{132 \mathrm{a}}$ A. Ventura, ${ }^{73 a, 73 b}$ D. Ventura ${ }^{86}$ M. Venturi, ${ }^{169}$ N. Venturi, ${ }^{158}$ A. Venturini, ${ }^{23}$ V. Vercesi, ${ }^{121 a}$ M. Verducci, ${ }^{132 a, 132 b}$ W. Verkerke, ${ }^{107}$ J. C. Vermeulen, ${ }^{107}$ A. Vest,${ }^{44}$ M. C. Vetterli, ${ }^{12, e}$ O. Viazlo, ${ }^{81}$ I. Vichou, ${ }^{165}$ T. Vickey, ${ }^{139}$ O. E. Vickey Boeriu, ${ }^{139}$ G. H. A. Viehhauser, ${ }^{120}$ S. Viel,${ }^{15}$ R. Vigne, ${ }^{62}$ M. Villa, ${ }^{20 a, 20 b}$ M. Villaplana Perez,${ }^{91 a, 91 b}$ E. Vilucchi ${ }^{47}$ M. G. Vincter, ${ }^{29}$ V. B. Vinogradov,${ }^{65}$ I. Vivarelli, ${ }^{149}$ F. Vives Vaque, ${ }^{3}$ S. Vlachos, ${ }^{10}$ D. Vladoiu, ${ }^{100}$ M. Vlasak, ${ }^{128}$ M. Vogel, ${ }^{32 a}$ P. Vokac, ${ }^{128}$ G. Volpi, ${ }^{124 a, 124 b}$ M. Volpi,${ }^{88}$ H. von der Schmitt, ${ }^{101}$ H. von Radziewski, ${ }^{48}$ E. von Toerne, ${ }^{21}$ V. Vorobel, ${ }^{129}$ K. Vorobev, ${ }^{98}$ M. Vos, ${ }^{167}$ R. Voss,${ }^{30}$ J. H. Vossebeld, ${ }^{74}$ N. Vranjes, ${ }^{13}$

M. Vranjes Milosavljevic, ${ }^{13}$ V. Vrba, ${ }^{127}$ M. Vreeswijk, ${ }^{107}$ R. Vuillermet, ${ }^{30}$ I. Vukotic,${ }^{31}$ Z. Vykydal, ${ }^{128}$ P. Wagner, ${ }^{21}$ W. Wagner, ${ }^{175}$ H. Wahlberg, ${ }^{71}$ S. Wahrmund, ${ }^{44}$ J. Wakabayashi, ${ }^{103}$ J. Walder, ${ }^{72}$ R. Walker, ${ }^{100}$ W. Walkowiak, ${ }^{141}$ C. Wang, ${ }^{33 c}$ F. Wang, ${ }^{173}$ H. Wang, ${ }^{15}$ H. Wang, ${ }^{40}$ J. Wang, ${ }^{42}$ J. Wang, ${ }^{33 a}$ K. Wang, ${ }^{87}$ R. Wang, ${ }^{6}$ S. M. Wang, ${ }^{151}$ T. Wang, ${ }^{21}$ X. Wang, ${ }^{176}$ C. Wanotayaroj, ${ }^{116}$ A. Warburton, ${ }^{87}$ C. P. Ward ${ }^{28}$ D. R. Wardrope,${ }^{78}$ M. Warsinsky, ${ }^{48}$ A. Washbrook, ${ }^{46}$ C. Wasicki, ${ }^{42}$ P. M. Watkins, ${ }^{18}$ A. T. Watson, ${ }^{18}$ I. J. Watson, ${ }^{150}$ M. F. Watson, ${ }^{18}$ G. Watts, ${ }^{138}$ S. Watts, ${ }^{84}$ B. M. Waugh,${ }^{78}$ S. Webb,${ }^{84}$ M. S. Weber, ${ }^{17}$ S. W. Weber, ${ }^{174}$ J. S. Webster, ${ }^{31}$ A. R. Weidberg, ${ }^{120}$ B. Weinert, ${ }^{61}$ J. Weingarten, ${ }^{54}$ C. Weiser, ${ }^{48}$ H. Weits, ${ }^{107}$ P. S. Wells, ${ }^{30}$ T. Wenaus, ${ }^{25}$ T. Wengler,${ }^{30}$ S. Wenig, ${ }^{30}$ N. Wermes,${ }^{21}$ M. Werner ${ }^{48}$ P. Werner, ${ }^{30}$ M. Wessels, ${ }^{58 a}$ J. Wetter, ${ }^{161}$ K. Whalen, ${ }^{116}$ A. M. Wharton, ${ }^{72}$ A. White, ${ }^{8}$ M. J. White, ${ }^{1}$ R. White, ${ }^{32 b}$ S. White, ${ }^{124 a, 124 b}$ D. Whiteson, ${ }^{163}$ F. J. Wickens, ${ }^{131}$

W. Wiedenmann, ${ }^{173}$ M. Wielers, ${ }^{131}$ P. Wienemann, ${ }^{21}$ C. Wiglesworth,${ }^{36}$ L. A. M. Wiik-Fuchs, ${ }^{21}$ A. Wildauer, ${ }^{101}$

H. G. Wilkens, ${ }^{30}$ H. H. Williams, ${ }^{122}$ S. Williams, ${ }^{107}$ C. Willis, ${ }^{90}$ S. Willocq ${ }^{86}$ A. Wilson, ${ }^{89}$ J. A. Wilson, ${ }^{18}$

I. Wingerter-Seez, ${ }^{5}$ F. Winklmeier, ${ }^{116}$ B. T. Winter, ${ }^{21}$ M. Wittgen, ${ }^{143}$ J. Wittkowski, ${ }^{100}$ S. J. Wollstadt, ${ }^{83}$ M. W. Wolter, ${ }^{39}$ H. Wolters, ${ }^{126 a, 126 c}$ B. K. Wosiek, ${ }^{39}$ J. Wotschack, ${ }^{30}$ M. J. Woudstra, ${ }^{84}$ K. W. Wozniak, ${ }^{39}$ M. Wu, ${ }^{55}$ M. Wu ${ }^{31}$ S. L. Wu, ${ }^{173}$ X. Wu, ${ }^{49}$ Y. Wu ${ }^{89}$ T. R. Wyatt, ${ }^{84}$ B. M. Wynne, ${ }^{46}$ S. Xella, ${ }^{36}$ D. Xu, ${ }^{33 a}$ L. Xu,${ }^{33 b, 11}$ B. Yabsley, ${ }^{150}$ S. Yacoob, ${ }^{145 a}$ R. Yakabe,${ }^{67}$ M. Yamada, ${ }^{66}$ Y. Yamaguchi, ${ }^{118}$ A. Yamamoto ${ }^{66}$ S. Yamamoto, ${ }^{155}$ T. Yamanaka, ${ }^{155}$ K. Yamauchi, ${ }^{103}$ Y. Yamazaki, ${ }^{67}$ Z. Yan, ${ }^{22}$ H. Yang, ${ }^{33 e}$ H. Yang, ${ }^{173}$ Y. Yang, ${ }^{151}$ W-M. Yao, ${ }^{15}$ Y. Yasu, ${ }^{66}$ E. Yatsenko, ${ }^{5}$ K. H. Yau Wong, ${ }^{21}$ J. Ye, ${ }^{40}$ S. Ye,${ }^{25}$ I. Yeletskikh, ${ }^{65}$ A. L. Yen, ${ }^{57}$ E. Yildirim, ${ }^{42}$ K. Yorita, ${ }^{171}$ R. Yoshida, ${ }^{6}$ K. Yoshihara, ${ }^{122}$ C. Young, ${ }^{143}$ C. J. S. Young, ${ }^{30}$ S. Youssef, ${ }^{22}$ D. R. Yu, ${ }^{15}$ J. Yu, ${ }^{8}$ J. M. Yu, ${ }^{89}$ J. Yu, ${ }^{114}$ L. Yuan, ${ }^{67}$ A. Yurkewicz, ${ }^{108}$ I. Yusuff, ${ }^{28, m m}$ B. Zabinski, ${ }^{39}$ R. Zaidan, ${ }^{63}$ A. M. Zaitsev, ${ }^{130, c c}$ J. Zalieckas, ${ }^{14}$ A. Zaman, ${ }^{148}$ S. Zambito, ${ }^{57}$ L. Zanello, ${ }^{132 a, 132 b}$ D. Zanzi, ${ }^{88}$ C. Zeitnitz, ${ }^{175}$ M. Zeman, ${ }^{128}$ A. Zemla ${ }^{38 a}$ K. Zengel, ${ }^{23}$ O. Zenin ${ }^{130}$ T. Ženiš, ${ }^{144 a}$ D. Zerwas, ${ }^{117}$ D. Zhang, ${ }^{89}$ F. Zhang, ${ }^{173}$ H. Zhang, ${ }^{33 c}$ J. Zhang, ${ }^{6}$ L. Zhang, ${ }^{48}$ R. Zhang, ${ }^{33 b}$ X. Zhang, ${ }^{33 d}$ Z. Zhang, ${ }^{117}$ X. Zhao, ${ }^{40}$ Y. Zhao, ${ }^{33 d, 117}$ Z. Zhao, ${ }^{33 b}$ A. Zhemchugov, ${ }^{65}$ J. Zhong, ${ }^{120}$ B. Zhou, ${ }^{89}$ C. Zhou, ${ }^{45}$ L. Zhou, ${ }^{35}$ L. Zhou, ${ }^{40}$ N. Zhou, ${ }^{163}$ C. G. Zhu, ${ }^{33 d}$ H. Zhu, ${ }^{33 a}$ J. Zhu, ${ }^{89}$ Y. Zhu, ${ }^{33 b}$ X. Zhuang, ${ }^{33 a}$ K. Zhukov, ${ }^{96}$ A. Zibell, ${ }^{174}$ D. Zieminska, ${ }^{61}$ N. I. Zimine, ${ }^{65}$ C. Zimmermann, ${ }^{83}$ S. Zimmermann, ${ }^{48}$ Z. Zinonos, ${ }^{54}$ M. Zinser,${ }^{83}$ M. Ziolkowski, ${ }^{141}$ L. Živković, ${ }^{13}$ G. Zobernig, ${ }^{173}$ A. Zoccoli, ${ }^{20 a, 20 b}$ M. zur Nedden, ${ }^{16}$ G. Zurzolo ${ }^{104 a, 104 b}$ and L. Zwalinski ${ }^{30}$

\title{
(ATLAS Collaboration)
}

\author{
${ }^{1}$ Department of Physics, University of Adelaide, Adelaide, Australia \\ ${ }^{2}$ Physics Department, SUNY Albany, Albany New York, USA \\ ${ }^{3}$ Department of Physics, University of Alberta, Edmonton AB, Canada \\ ${ }^{4 a}$ Department of Physics, Ankara University, Ankara, Turkey \\ ${ }^{4 \mathrm{~b}}$ Istanbul Aydin University, Istanbul, Turkey \\ ${ }^{4 \mathrm{c}}$ Division of Physics, TOBB University of Economics and Technology, Ankara, Turkey \\ ${ }^{5}$ LAPP, CNRS/IN2P3 and Université Savoie Mont Blanc, Annecy-le-Vieux, France \\ ${ }^{6}$ High Energy Physics Division, Argonne National Laboratory, Argonne Illinois, USA \\ ${ }^{7}$ Department of Physics, University of Arizona, Tucson Arizona, USA \\ ${ }^{8}$ Department of Physics, University of Texas at Arlington, Arlington Texas, USA
}


${ }^{9}$ Physics Department, University of Athens, Athens, Greece

${ }^{10}$ Physics Department, National Technical University of Athens, Zografou, Greece

${ }^{11}$ Institute of Physics, Azerbaijan Academy of Sciences, Baku, Azerbaijan

${ }^{12}$ Institut de Física d'Altes Energies and Departament de Física de la Universitat Autònoma de Barcelona, Barcelona, Spain

${ }^{13}$ Institute of Physics, University of Belgrade, Belgrade, Serbia

${ }^{14}$ Department for Physics and Technology, University of Bergen, Bergen, Norway

${ }^{15}$ Physics Division, Lawrence Berkeley National Laboratory and University of California, Berkeley California, USA

${ }^{16}$ Department of Physics, Humboldt University, Berlin, Germany

${ }^{17}$ Albert Einstein Center for Fundamental Physics and Laboratory for High Energy Physics, University of Bern, Bern, Switzerland

${ }^{18}$ School of Physics and Astronomy, University of Birmingham, Birmingham, United Kingdom

${ }^{19 a}$ Department of Physics, Bogazici University, Istanbul, Turkey

${ }^{19 b}$ Department of Physics, Dogus University, Istanbul, Turkey

${ }^{19 \mathrm{c}}$ Department of Physics Engineering, Gaziantep University, Gaziantep, Turkey ${ }^{20 \mathrm{a}}$ INFN Sezione di Bologna, Italy

${ }^{20 \mathrm{~b}}$ Dipartimento di Fisica e Astronomia, Università di Bologna, Bologna, Italy

${ }^{21}$ Physikalisches Institut, University of Bonn, Bonn, Germany

${ }^{22}$ Department of Physics, Boston University, Boston Massachusetts, USA

${ }^{23}$ Department of Physics, Brandeis University, Waltham Massachusetts, USA

${ }^{24 a}$ Universidade Federal do Rio De Janeiro COPPE/EE/IF, Rio de Janeiro, Brazil

${ }^{24 \mathrm{~b}}$ Electrical Circuits Department, Federal University of Juiz de Fora (UFJF), Juiz de Fora, Brazil

${ }^{24 \mathrm{c}}$ Federal University of Sao Joao del Rei (UFSJ), Sao Joao del Rei, Brazil

${ }^{24 \mathrm{~d}}$ Instituto de Fisica, Universidade de Sao Paulo, Sao Paulo, Brazil

${ }^{25}$ Physics Department, Brookhaven National Laboratory, Upton New York, USA

${ }^{26 a}$ National Institute of Physics and Nuclear Engineering, Bucharest, Romania

${ }^{26 \mathrm{~b}}$ National Institute for Research and Development of Isotopic and Molecular Technologies, Physics Department, Cluj Napoca, Romania

${ }^{26 c}$ University Politehnica Bucharest, Bucharest, Romania

${ }^{26 \mathrm{~d}}$ West University in Timisoara, Timisoara, Romania

${ }^{27}$ Departamento de Física, Universidad de Buenos Aires, Buenos Aires, Argentina

${ }^{28}$ Cavendish Laboratory, University of Cambridge, Cambridge, United Kingdom

${ }^{29}$ Department of Physics, Carleton University, Ottawa ON, Canada

${ }^{30}$ CERN, Geneva, Switzerland

${ }^{31}$ Enrico Fermi Institute, University of Chicago, Chicago Illinois, USA

${ }^{32 a}$ Departamento de Física, Pontificia Universidad Católica de Chile, Santiago, Chile

${ }^{32 \mathrm{~b}}$ Departamento de Física, Universidad Técnica Federico Santa María, Valparaíso, Chile

${ }^{33 a}$ Institute of High Energy Physics, Chinese Academy of Sciences, Beijing, China

${ }^{33 b}$ Department of Modern Physics, University of Science and Technology of China, Anhui, China

${ }^{33 \mathrm{c}}$ Department of Physics, Nanjing University, Jiangsu, China

${ }^{33 \mathrm{~d}}$ School of Physics, Shandong University, Shandong, China

${ }^{33 \mathrm{e}}$ Department of Physics and Astronomy, Shanghai Key Laboratory for Particle Physics and Cosmology, Shanghai Jiao Tong University, Shanghai, China

${ }^{33 \mathrm{f}}$ Physics Department, Tsinghua University, Beijing 100084, China

${ }^{34}$ Laboratoire de Physique Corpusculaire, Clermont Université and Université Blaise Pascal and CNRS/IN2P3, Clermont-Ferrand, France

${ }^{35}$ Nevis Laboratory, Columbia University, Irvington New York, USA

${ }^{36}$ Niels Bohr Institute, University of Copenhagen, Kobenhavn, Denmark

${ }^{37}$ INFN Gruppo Collegato di Cosenza, Laboratori Nazionali di Frascati, Italy

${ }^{37 \mathrm{~b}}$ Dipartimento di Fisica, Università della Calabria, Rende, Italy

${ }^{38 \mathrm{a}}$ AGH University of Science and Technology, Faculty of Physics and Applied Computer Science, Krakow, Poland

${ }^{38 \mathrm{~b}}$ Marian Smoluchowski Institute of Physics, Jagiellonian University, Krakow, Poland

${ }^{39}$ Institute of Nuclear Physics Polish Academy of Sciences, Krakow, Poland

${ }^{40}$ Physics Department, Southern Methodist University, Dallas Texas, USA

${ }^{41}$ Physics Department, University of Texas at Dallas, Richardson Texas, USA

${ }^{42}$ DESY, Hamburg and Zeuthen, Germany

${ }^{43}$ Institut für Experimentelle Physik IV, Technische Universität Dortmund, Dortmund, Germany

${ }^{44}$ Institut für Kern- und Teilchenphysik, Technische Universität Dresden, Dresden, Germany 
${ }^{45}$ Department of Physics, Duke University, Durham North Carolina, USA

${ }^{46}$ SUPA-School of Physics and Astronomy, University of Edinburgh, Edinburgh, United Kingdom

${ }^{47}$ INFN Laboratori Nazionali di Frascati, Frascati, Italy

${ }^{48}$ Fakultät für Mathematik und Physik, Albert-Ludwigs-Universität, Freiburg, Germany

${ }^{49}$ Section de Physique, Université de Genève, Geneva, Switzerland

${ }^{50 a}$ INFN Sezione di Genova, Italy

${ }^{50 \mathrm{~b}}$ Dipartimento di Fisica, Università di Genova, Genova, Italy

${ }^{51 a}$ E. Andronikashvili Institute of Physics, Iv. Javakhishvili Tbilisi State University, Tbilisi, Georgia

${ }^{51 b}$ High Energy Physics Institute, Tbilisi State University, Tbilisi, Georgia

${ }^{52}$ II Physikalisches Institut, Justus-Liebig-Universität Giessen, Giessen, Germany

${ }^{53}$ SUPA-School of Physics and Astronomy, University of Glasgow, Glasgow, United Kingdom

${ }^{54}$ II Physikalisches Institut, Georg-August-Universität, Göttingen, Germany

${ }^{55}$ Laboratoire de Physique Subatomique et de Cosmologie, Université Grenoble-Alpes, CNRS/IN2P3, Grenoble, France

${ }^{56}$ Department of Physics, Hampton University, Hampton Virginia, USA

${ }^{57}$ Laboratory for Particle Physics and Cosmology, Harvard University, Cambridge Massachusetts, USA

${ }^{58 a}$ Kirchhoff-Institut für Physik, Ruprecht-Karls-Universität Heidelberg, Heidelberg, Germany

${ }^{58 \mathrm{~b}}$ Physikalisches Institut, Ruprecht-Karls-Universität Heidelberg, Heidelberg, Germany

${ }^{58 \mathrm{c}}$ ZITI Institut für technische Informatik, Ruprecht-Karls-Universität Heidelberg, Mannheim, Germany

${ }^{59}$ Faculty of Applied Information Science, Hiroshima Institute of Technology, Hiroshima, Japan

${ }^{60 a}$ Department of Physics, Chinese University of Hong Kong, Shatin, N.T., Hong Kong, China

${ }^{60 \mathrm{~b}}$ Department of Physics, University of Hong Kong, Hong Kong, China

${ }^{60 c}$ Department of Physics, Hong Kong University of Science and Technology, Clear Water Bay, Kowloon, Hong Kong, China

${ }^{61}$ Department of Physics, Indiana University, Bloomington Indiana, USA

${ }^{62}$ Institut für Astro- und Teilchenphysik, Leopold-Franzens-Universität, Innsbruck, Austria

${ }^{63}$ University of Iowa, Iowa City Iowa, USA

${ }^{64}$ Department of Physics and Astronomy, Iowa State University, Ames Iowa, USA

${ }^{65}$ Joint Institute for Nuclear Research, JINR Dubna, Dubna, Russia

${ }^{66}$ KEK, High Energy Accelerator Research Organization, Tsukuba, Japan

${ }^{67}$ Graduate School of Science, Kobe University, Kobe, Japan

${ }^{68}$ Faculty of Science, Kyoto University, Kyoto, Japan

${ }^{69}$ Kyoto University of Education, Kyoto, Japan

${ }^{70}$ Department of Physics, Kyushu University, Fukuoka, Japan

${ }^{71}$ Instituto de Física La Plata, Universidad Nacional de La Plata and CONICET, La Plata, Argentina

${ }^{72}$ Physics Department, Lancaster University, Lancaster, United Kingdom

${ }^{73 \mathrm{a}}$ INFN Sezione di Lecce, Italy

${ }^{73 b}$ Dipartimento di Matematica e Fisica, Università del Salento, Lecce, Italy

${ }^{74}$ Oliver Lodge Laboratory, University of Liverpool, Liverpool, United Kingdom

${ }^{75}$ Department of Physics, Jožef Stefan Institute and University of Ljubljana, Ljubljana, Slovenia

${ }^{76}$ School of Physics and Astronomy, Queen Mary University of London, London, United Kingdom

${ }^{77}$ Department of Physics, Royal Holloway University of London, Surrey, United Kingdom

${ }^{78}$ Department of Physics and Astronomy, University College London, London, United Kingdom

${ }^{79}$ Louisiana Tech University, Ruston Los Angeles, USA

${ }^{80}$ Laboratoire de Physique Nucléaire et de Hautes Energies, UPMC and Université Paris-Diderot and CNRS/IN2P3, Paris, France

${ }^{81}$ Fysiska institutionen, Lunds universitet, Lund, Sweden

${ }^{82}$ Departamento de Fisica Teorica C-15, Universidad Autonoma de Madrid, Madrid, Spain

${ }^{83}$ Institut für Physik, Universität Mainz, Mainz, Germany

${ }^{84}$ School of Physics and Astronomy, University of Manchester, Manchester, United Kingdom

${ }^{85}$ CPPM, Aix-Marseille Université and CNRS/IN2P3, Marseille, France

${ }^{86}$ Department of Physics, University of Massachusetts, Amherst Massachusetts, USA

${ }^{87}$ Department of Physics, McGill University, Montreal QC, Canada

${ }^{88}$ School of Physics, University of Melbourne, Victoria, Australia

${ }^{89}$ Department of Physics, University of Michigan, Ann Arbor Michigan, USA

${ }^{90}$ Department of Physics and Astronomy, Michigan State University, East Lansing Michigan, USA

${ }^{91 \mathrm{a}}$ INFN Sezione di Milano, Italy

${ }^{91 \mathrm{~b}}$ Dipartimento di Fisica, Università di Milano, Milano, Italy

${ }^{92}$ B.I. Stepanov Institute of Physics, National Academy of Sciences of Belarus, Minsk, Republic of Belarus 
${ }^{93}$ National Scientific and Educational Centre for Particle and High Energy Physics, Minsk, Republic of Belarus

${ }^{94}$ Department of Physics, Massachusetts Institute of Technology, Cambridge Massachusetts, USA

${ }^{95}$ Group of Particle Physics, University of Montreal, Montreal QC, Canada

${ }^{96}$ P.N. Lebedev Institute of Physics, Academy of Sciences, Moscow, Russia

${ }^{97}$ Institute for Theoretical and Experimental Physics (ITEP), Moscow, Russia

${ }^{98}$ National Research Nuclear University MEPhI, Moscow, Russia

${ }^{99}$ D.V. Skobeltsyn Institute of Nuclear Physics, M.V. Lomonosov Moscow State University, Moscow, Russia

${ }^{100}$ Fakultät für Physik, Ludwig-Maximilians-Universität München, München, Germany

${ }^{101}$ Max-Planck-Institut für Physik (Werner-Heisenberg-Institut), München, Germany

${ }^{102}$ Nagasaki Institute of Applied Science, Nagasaki, Japan

${ }^{103}$ Graduate School of Science and Kobayashi-Maskawa Institute, Nagoya University, Nagoya, Japan

${ }^{104 a}$ INFN Sezione di Napoli, Italy

${ }^{104 \mathrm{~b}}$ Dipartimento di Fisica, Università di Napoli, Napoli, Italy

${ }^{105}$ Department of Physics and Astronomy, University of New Mexico, Albuquerque New Mexico, USA

${ }^{106}$ Institute for Mathematics, Astrophysics and Particle Physics, Radboud University Nijmegen/Nikhef, Nijmegen, Netherlands

${ }^{107}$ Nikhef National Institute for Subatomic Physics and University of Amsterdam, Amsterdam, Netherlands

${ }^{108}$ Department of Physics, Northern Illinois University, DeKalb Illinois, USA

${ }^{109}$ Budker Institute of Nuclear Physics, SB RAS, Novosibirsk, Russia

${ }^{110}$ Department of Physics, New York University, New York New York, USA

${ }^{111}$ Ohio State University, Columbus Ohio, USA

${ }^{112}$ Faculty of Science, Okayama University, Okayama, Japan

${ }^{113}$ Homer L. Dodge Department of Physics and Astronomy, University of Oklahoma, Norman Oklahoma, USA

${ }^{114}$ Department of Physics, Oklahoma State University, Stillwater Oklahoma, USA

${ }^{115}$ Palacký University, RCPTM, Olomouc, Czech Republic

${ }^{116}$ Center for High Energy Physics, University of Oregon, Eugene Oregon, USA

${ }^{117}$ LAL, Université Paris-Sud and CNRS/IN2P3, Orsay, France

${ }^{118}$ Graduate School of Science, Osaka University, Osaka, Japan

${ }^{119}$ Department of Physics, University of Oslo, Oslo, Norway

${ }^{120}$ Department of Physics, Oxford University, Oxford, United Kingdom

${ }^{121 a}$ INFN Sezione di Pavia, Italy

${ }^{121 \mathrm{~b}}$ Dipartimento di Fisica, Università di Pavia, Pavia, Italy

${ }^{122}$ Department of Physics, University of Pennsylvania, Philadelphia Pennsylvania, USA

${ }^{123}$ National Research Centre "Kurchatov Institute" B.P.Konstantinov Petersburg Nuclear Physics Institute, St. Petersburg, Russia

${ }^{124 a}$ INFN Sezione di Pisa, Italy

${ }^{124 \mathrm{~b}}$ Dipartimento di Fisica E.Fermi, Università di Pisa, Pisa, Italy

${ }^{125}$ Department of Physics and Astronomy, University of Pittsburgh, Pittsburgh Pennsylvania, USA

${ }^{126 a}$ Laboratorio de Instrumentacao e Fisica Experimental de Particulas-LIP, Lisboa, Portugal

${ }^{126 \mathrm{~b}}$ Faculdade de Ciências, Universidade de Lisboa, Lisboa, Portugal

${ }^{126 c}$ Department of Physics, University of Coimbra, Coimbra, Portugal

${ }^{126 \mathrm{~d}}$ Centro de Física Nuclear da Universidade de Lisboa, Lisboa, Portugal

${ }^{126 e}$ Departamento de Fisica, Universidade do Minho, Braga, Portugal

${ }^{126 \mathrm{f}}$ Departamento de Fisica Teorica y del Cosmos and CAFPE, Universidad de Granada, Granada (Spain), Portugal

${ }^{126 \mathrm{~g}}$ Dep Fisica and CEFITEC of Faculdade de Ciencias e Tecnologia, Universidade Nova de Lisboa, Caparica, Portugal

${ }^{127}$ Institute of Physics, Academy of Sciences of the Czech Republic, Praha, Czech Republic

${ }^{128}$ Czech Technical University in Prague, Praha, Czech Republic

${ }^{129}$ Faculty of Mathematics and Physics, Charles University in Prague, Praha, Czech Republic

${ }^{130}$ State Research Center Institute for High Energy Physics, Protvino, Russia

${ }^{131}$ Particle Physics Department, Rutherford Appleton Laboratory, Didcot, United Kingdom

${ }^{132 a}$ INFN Sezione di Roma, Italy

${ }^{132 \mathrm{~b}}$ Dipartimento di Fisica, Sapienza Università di Roma, Roma, Italy

${ }^{133 a}$ INFN Sezione di Roma Tor Vergata, Italy

${ }^{133 \mathrm{~b}}$ Dipartimento di Fisica, Università di Roma Tor Vergata, Roma, Italy

${ }^{134 a}$ INFN Sezione di Roma Tre, Italy

${ }^{134 \mathrm{~b}}$ Dipartimento di Matematica e Fisica, Università Roma Tre, Roma, Italy 
${ }^{135 a}$ Faculté des Sciences Ain Chock, Réseau Universitaire de Physique des Hautes Energies-Université Hassan II, Casablanca, Morocco

${ }^{135 \mathrm{~b}}$ Centre National de l'Energie des Sciences Techniques Nucleaires, Rabat, Morocco

${ }^{135 \mathrm{c}}$ Faculté des Sciences Semlalia, Université Cadi Ayyad, LPHEA-Marrakech, Morocco

${ }^{135 \mathrm{~d}}$ Faculté des Sciences, Université Mohamed Premier and LPTPM, Oujda, Morocco

${ }^{135 \mathrm{e}}$ Faculté des sciences, Université Mohammed V-Agdal, Rabat, Morocco

${ }^{136}$ DSM/IRFU (Institut de Recherches sur les Lois Fondamentales de l'Univers), CEA Saclay

(Commissariat à l'Energie Atomique et aux Energies Alternatives), Gif-sur-Yvette, France

${ }^{137}$ Santa Cruz Institute for Particle Physics, University of California Santa Cruz, Santa Cruz California, USA

${ }^{138}$ Department of Physics, University of Washington, Seattle Washington, USA

${ }^{139}$ Department of Physics and Astronomy, University of Sheffield, Sheffield, United Kingdom

${ }^{140}$ Department of Physics, Shinshu University, Nagano, Japan

${ }^{141}$ Fachbereich Physik, Universität Siegen, Siegen, Germany

${ }^{142}$ Department of Physics, Simon Fraser University, Burnaby BC, Canada

${ }^{143}$ SLAC National Accelerator Laboratory, Stanford California, USA

${ }^{144 a}$ Faculty of Mathematics, Physics \& Informatics, Comenius University, Bratislava, Slovak Republic ${ }^{144 \mathrm{~b}}$ Department of Subnuclear Physics, Institute of Experimental Physics of the Slovak Academy of Sciences, Kosice, Slovak Republic

${ }^{145 a}$ Department of Physics, University of Cape Town, Cape Town, South Africa

${ }^{145 \mathrm{~b}}$ Department of Physics, University of Johannesburg, Johannesburg, South Africa

${ }^{145 c}$ School of Physics, University of the Witwatersrand, Johannesburg, South Africa

${ }^{146 a}$ Department of Physics, Stockholm University, Sweden

${ }^{146 \mathrm{~b}}$ The Oskar Klein Centre, Stockholm, Sweden

${ }^{147}$ Physics Department, Royal Institute of Technology, Stockholm, Sweden

${ }^{148}$ Departments of Physics \& Astronomy and Chemistry, Stony Brook University, Stony Brook New York, USA

${ }^{149}$ Department of Physics and Astronomy, University of Sussex, Brighton, United Kingdom

${ }^{150}$ School of Physics, University of Sydney, Sydney, Australia

${ }^{151}$ Institute of Physics, Academia Sinica, Taipei, Taiwan

${ }^{152}$ Department of Physics, Technion: Israel Institute of Technology, Haifa, Israel

${ }^{153}$ Raymond and Beverly Sackler School of Physics and Astronomy, Tel Aviv University, Tel Aviv, Israel

${ }^{154}$ Department of Physics, Aristotle University of Thessaloniki, Thessaloniki, Greece

${ }^{155}$ International Center for Elementary Particle Physics and Department of Physics, The University of Tokyo, Tokyo, Japan

${ }^{156}$ Graduate School of Science and Technology, Tokyo Metropolitan University, Tokyo, Japan

${ }^{157}$ Department of Physics, Tokyo Institute of Technology, Tokyo, Japan

${ }^{158}$ Department of Physics, University of Toronto, Toronto ON, Canada

${ }^{159 a}$ TRIUMF, Vancouver BC, Canada

${ }^{159 \mathrm{~b}}$ Department of Physics and Astronomy, York University, Toronto ON, Canada

${ }^{160}$ Faculty of Pure and Applied Sciences, University of Tsukuba, Tsukuba, Japan

${ }^{161}$ Department of Physics and Astronomy, Tufts University, Medford Massachusetts, USA

${ }^{162}$ Centro de Investigaciones, Universidad Antonio Narino, Bogota, Colombia

${ }^{163}$ Department of Physics and Astronomy, University of California Irvine, Irvine California, USA

${ }^{164 a}$ INFN Gruppo Collegato di Udine, Sezione di Trieste, Udine, Italy

${ }^{164 \mathrm{~b}}$ ICTP, Trieste, Italy

${ }^{164 c}$ Dipartimento di Chimica, Fisica e Ambiente, Università di Udine, Udine, Italy

${ }^{165}$ Department of Physics, University of Illinois, Urbana Illinois, USA

${ }^{166}$ Department of Physics and Astronomy, University of Uppsala, Uppsala, Sweden

${ }^{167}$ Instituto de Física Corpuscular (IFIC) and Departamento de Física Atómica, Molecular y Nuclear and

Departamento de Ingeniería Electrónica and Instituto de Microelectrónica de Barcelona (IMB-CNM),

University of Valencia and CSIC, Valencia, Spain

${ }^{168}$ Department of Physics, University of British Columbia, Vancouver BC, Canada

${ }^{169}$ Department of Physics and Astronomy, University of Victoria, Victoria BC, Canada

${ }^{170}$ Department of Physics, University of Warwick, Coventry, United Kingdom

${ }^{171}$ Waseda University, Tokyo, Japan

${ }^{172}$ Department of Particle Physics, Weizmann Institute of Science, Rehovot, Israel

${ }^{173}$ Department of Physics, University of Wisconsin, Madison Wisconsin, USA

${ }^{174}$ Fakultät für Physik und Astronomie, Julius-Maximilians-Universität, Würzburg, Germany

${ }^{175}$ Fachbereich C Physik, Bergische Universität Wuppertal, Wuppertal, Germany 
${ }^{176}$ Department of Physics, Yale University, New Haven Connecticut, USA

${ }^{177}$ Yerevan Physics Institute, Yerevan, Armenia

${ }^{178}$ Centre de Calcul de l'Institut National de Physique Nucléaire et de Physique des Particules (IN2P3), Villeurbanne, France

\footnotetext{
${ }^{a}$ Deceased.

${ }^{\mathrm{b}}$ Also at Department of Physics, King's College London, London, United Kingdom.

${ }^{c}$ Also at Institute of Physics, Azerbaijan Academy of Sciences, Baku, Azerbaijan.

${ }^{\mathrm{d}}$ Also at Novosibirsk State University, Novosibirsk, Russia.

${ }^{\mathrm{e}}$ Also at TRIUMF, Vancouver BC, Canada.

${ }^{\mathrm{f}}$ Also at Department of Physics, California State University, Fresno CA, USA.

${ }^{g}$ Also at Department of Physics, University of Fribourg, Fribourg, Switzerland.

${ }^{\mathrm{h}}$ Also at Departamento de Fisica e Astronomia, Faculdade de Ciencias, Universidade do Porto, Portugal.

${ }^{1}$ Also at Tomsk State University, Tomsk, Russia.

${ }^{\mathrm{j}}$ Also at CPPM, Aix-Marseille Université and CNRS/IN2P3, Marseille, France.

${ }^{\mathrm{k}}$ Also at Universita di Napoli Parthenope, Napoli, Italy.

${ }^{1}$ Also at Institute of Particle Physics (IPP), Canada.

${ }^{\mathrm{m}}$ Also at Particle Physics Department, Rutherford Appleton Laboratory, Didcot, United Kingdom.

${ }^{\mathrm{n}}$ Also at Department of Physics, St. Petersburg State Polytechnical University, St. Petersburg, Russia.

${ }^{\circ}$ Also at Louisiana Tech University, Ruston LA, USA.

${ }^{\mathrm{p}}$ Also at Institucio Catalana de Recerca i Estudis Avancats, ICREA, Barcelona, Spain.

${ }^{\mathrm{q}}$ Also at Department of Physics, National Tsing Hua University, Taiwan.

${ }^{\mathrm{r}}$ Also at Department of Physics, The University of Texas at Austin, Austin TX, USA.

${ }^{\mathrm{s}}$ Also at Institute of Theoretical Physics, Ilia State University, Tbilisi, Georgia.

${ }^{\mathrm{t}}$ Also at CERN, Geneva, Switzerland.

"Also at Georgian Technical University (GTU),Tbilisi, Georgia.

${ }^{v}$ Also at Ochadai Academic Production, Ochanomizu University, Tokyo, Japan.

${ }^{\mathrm{w}}$ Also at Manhattan College, New York NY, USA.

${ }^{\mathrm{x}}$ Also at Hellenic Open University, Patras, Greece.

${ }^{y}$ Also at Institute of Physics, Academia Sinica, Taipei, Taiwan.

${ }^{\mathrm{z}}$ Also at LAL, Université Paris-Sud and CNRS/IN2P3, Orsay, France.

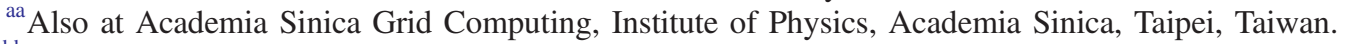

${ }^{\mathrm{bb}}$ Also at School of Physics, Shandong University, Shandong, China.

${ }^{\mathrm{cc}}$ Also at Moscow Institute of Physics and Technology State University, Dolgoprudny, Russia.

${ }^{\mathrm{dd}}$ Also at Section de Physique, Université de Genève, Geneva, Switzerland.

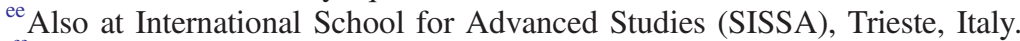

${ }^{\mathrm{ff}}$ Also at Department of Physics and Astronomy, University of South Carolina, Columbia SC, USA.

${ }^{\mathrm{gg}}$ Also at School of Physics and Engineering, Sun Yat-sen University, Guangzhou, China.

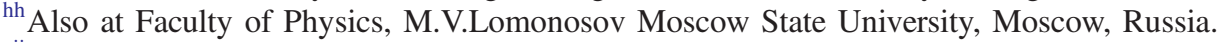

${ }^{i i}$ Also at National Research Nuclear University MEPhI, Moscow, Russia.

${ }^{\mathrm{jj}}$ Also at Department of Physics, Stanford University, Stanford CA, USA.

${ }^{\mathrm{kk}}$ Also at Institute for Particle and Nuclear Physics, Wigner Research Centre for Physics, Budapest, Hungary.

${ }^{11}$ Also at Department of Physics, The University of Michigan, Ann Arbor MI, USA.

${ }^{\mathrm{mm}}$ Also at University of Malaya, Department of Physics, Kuala Lumpur, Malaysia.
} 